\title{
Probiotic Lacticaseibacillus rhamnosus HA-114 suppresses age-dependent neurodegeneration via mitochondrial beta-oxidation
}

\section{Audrey Labarre}

Centre Hospitalier de l'Université de Montréal

\section{Ericka Guitard}

Centre Hospitalier de l'Université de Montréal

\section{Gilles Tossing}

Centre Hospitalier de l'Université de Montréal

\section{Eric Bareke}

Centre Hospitalier de l'Université de Montréal

Marjorie Labrecque

Centre Hospitalier de l'Université de Montréal

\section{Martine Tetreault}

University of Montreal

\section{Alex Parker ( $\nabla$ ja.parker@umontreal.ca )}

Centre Hospitalier de l'Université de Montréal https://orcid.org/0000-0002-3333-2445

\section{Article}

\section{Keywords:}

Posted Date: September 24th, 2020

DOl: https://doi.org/10.21203/rs.3.rs-59192/v1

License: (c) (i) This work is licensed under a Creative Commons Attribution 4.0 International License. Read Full License 


\title{
Probiotic Lacticaseibacillus rhamnosus HA-114 suppresses age-dependent neurodegeneration via mitochondrial $\beta$-oxidation
}

\author{
Authors: A. Labarre ${ }^{1,2}$, E. Guitard ${ }^{1,2}$, G. Tossing ${ }^{1,2}$, Eric Bareke2 ${ }^{2}$, Marjorie \\ Labrecque $^{2,3}$, Martine Tétreault ${ }^{1,2}$ \& JA Parker ${ }^{1,2}$ *
}

\section{Affiliations:}

${ }^{1}$ Département de neurosciences, Université de Montréal, Montréal, Canada

${ }^{2}$ Centre de recherche du centre hospitalier de l'Université de Montréal (CRCHUM), Montréal, Canada.

${ }^{3}$ Département de biochimie, Université de Montréal, Montréal, Canada

*Correspondence to: ja.parker@umontreal.ca 


\begin{abstract}
The human microbiota is believed to influence health. Microbiome dysbiosis may be linked to neurological conditions like Alzheimer's disease (AD), amyotrophic lateral sclerosis (ALS) and Huntington's disease (HD). We report the ability of a probiotic bacterial strain in reversing neurodegeneration phenotypes. We show that Lacticaseibacillus rhamnosus HA-114 is neuroprotective in C. elegans models of ALS and HD. Our results show that neuroprotection from $L$. rhamnosus HA-114 is unique from other $L$. rhamnosus strains, and resides in its fatty acid content. Neuroprotection by L. rhamnosus HA-114 requires acdh-1/ACADSB, kat-1/ACAT1 and elo-6/ELOVL3/6, which are key fatty acid metabolism and mitochondrial $\beta$ oxidation genes. Moreover, L. rhamnosus HA-114 delayed disease onset and suppressed motor neuron degeneration in an aggressive mouse model of ALS. Our data suggest that disrupted lipid metabolism contributes to neurodegeneration and that dietary intervention with $L$. rhamnosus HA-114 restores lipid homeostasis and energy balance through mitochondrial $\beta$-oxidation. L. rhamnosus HA-114 is suitable for human consumption opening the possibility of modifying disease progression by dietary intervention.
\end{abstract}




\section{Introduction}

The human body is the natural habitat for many microbes, including hundreds of bacterial species referred to as the microbiota. A growing body of evidence demonstrates that gut microbiota is essential to human health, (Lynch and Pedersen, 2016) and a bacterial imbalance, termed dysbiosis, may be linked to many human diseases. It has been suggested that the oro-gastrointestinal tract microbiome may extend its effects beyond its niche and contribute systemically to various age-dependent diseases (Astafurov et al., 2014; Clark and Walker, 2017). Indeed, a growing number of studies have identified perturbations in gut microbiome for several neurodegenerative disorders, including amyotrophic lateral sclerosis (ALS), multiple sclerosis (MS), Alzheimer's (AD) and Parkinson's disease (PD) (Dopkins et al., 2018; Mulak and Bonaz, 2015; Vogt et al., 2017; Wu et al., 2015)

Therefore, positively regulating the microbiota-health relationship has attracted attention as preventative or therapeutic approach for many diseases, including neurodegenerative disorders (Roy Sarkar and Banerjee, 2019; Sherwin et al., 2018). Probiotic bacteria are defined as live microorganisms that may have positive health effects when consumed by the host. Emerging research has focused on using probiotic supplementation to counteract dysbiosis with the goal of stabilizing cognitive and emotional deficits in Alzheimer's disease (Akbari et al., 2016; Harding et al., 2017). However, the characterization of similar effects in amyotrophic lateral sclerosis (ALS) models awaits investigation. Of interest is are studies showing that antibiotic treatment to deplete the microbiome, or treatment with specific molecules derived from bacteria, like $\gamma$-butyrate, can delay the onset of phenotypes in ALS in hSOD1 ${ }^{\mathrm{G} 93 \mathrm{~A}}$ and C9orf72 loss-of-function mice (Blacher et al., 2019; Zhang et al., 2017; Burberry et al., 2020). However, insights into the molecular mechanisms underlying these effects are not fully understood.

Invertebrate model systems like $C$. elegans live in the presence of microbiota in natural and laboratory settings. Thanks to the wide range of genetic techniques available, $C$. elegans studies allow for direct connections to be made when the 
worms are grown with specific bacteria, facilitating the association between effects and genetic/molecular mechanisms. As such, C. elegans is now recognized as a suitable model to study effects of microbiota on life traits, gene expression, metabolic changes, and neuronal health (O'Donnell et al., 2020; ; Gerbaba et al., 2018; Schulenburg and Félix, 2017; Shapira, 2017; Watson et al., 2013; 2014). Furthermore, it is also an established model for investigating conserved genetic pathways that regulate the cellular stress response and neurodegeneration (Schmeisser and Parker, 2018; Therrien and Parker, 2014; Vérièpe et al., 2015).

We used the $C$. elegans model organism to screen for the effects of dietary supplementation of a panel of probiotic bacteria combinations on neurodegenerative phenotypes. We identified $L$. rhamnosus HA-114 as a bacterial strain that suppressed motor phenotypes and neurodegeneration in simple genetic models of ALS. A combination of genetics, genome profiling, behavioral analysis and microscopy highlighted lipid homeostasis disruption as a potential mechanism driving neurodegeneration. Moreover, we found that the beneficial effect of $L$. rhamnosus HA-114 could be extended to other genetic models of age-dependent neurodegeneration, including Huntington's disease (HD). We identified acdh-1 and kat-1, key genes in fatty acid metabolism and $\beta$-oxidation, as the core components of this neuroprotective mechanism. Altogether, these results demonstrate that a dietary probiotic intervention potentially modulating microbiota composition may regulate the neurodegeneration process and provide benefits to the host by restoring energy balance. 


\section{Results}

\section{Lacticaseibacillus rhamnosus HA-114 harbors neuroprotective activity in age-dependent models of neurodegeneration}

We previously created C. elegans ALS strains expressing full-length, untagged, human FUS or TDP-43 protein mutants in motor neurons, under the unc-47 promoter. Transgenic worms with motor neuron-specific expression of these mutant proteins show age-dependent paralysis and GABAergic degeneration at a rate significantly higher than wild-type FUS or TDP-43 expressing strains (Vaccaro et al., 2012). These phenotypes, associated with the disease, typically develop over 6-12 days of adulthood for worms cultured on petri plates and fed with E. coli OP50, their regular food source and main component of their natural microbiome in a laboratory setting. To investigate whether dietary probiotic interventions could modulate the phenotypes associated with ALS in our transgenic C. elegans models, we screened 16 different probiotic formulations, including 13 individual strains and 3 combinations (Table 1). We found that Lacticaseibacillus rhamnosus HA-114 rescued paralysis phenotypes on solid media in both of our ALS models, TDP-43 ${ }^{\text {A315T }}$ and FUS ${ }^{557}$, while having no significant effect on TDP-43 ${ }^{\text {WT }}$ and FUS ${ }^{W T}$ animals (Figure 1A and B). HA-114 was the only L. rhamnosus strain with the ability to prevent paralysis in FUS ${ }^{S 57 \Delta}$ worms (Figure S1A). Rescue of paralysis was also observed when worms were fed from day 6 of adulthood, at the usual onset of symptoms (Figure S1B). Next, we assessed whether the rescue of paralysis phenotypes was associated with GABAergic motor neuron function, and found a significant decrease in motor neuron degeneration in our transgenic animals compared to animals fed with control OP50 bacteria (Figure 1C-E). Since oxidative stress seems to play an important role in many neurodegenerative disorders, we wondered if $L$. rhamnosus HA-114 would be able to prevent damage related to chronic exposure to oxidative stress. We exposed our mutant FUS to $250 \mu \mathrm{M}$ paraquat over 12 days. Interestingly, chronic paraquat exposure increased paralysis phenotype in FUS ${ }^{557}$ animals fed with OP50, but not in animals fed with 
HA-114 (Figure S1C). These results suggest that L. rhamnosus HA-114 has the ability to overcome damage linked with chronic oxidative stress. However, HA-114 did not provide any lifespan extension in our FUS ${ }^{557 \Delta}$ worms (Figure S1D).

To determine whether the neuroprotective effect conferred by $L$. rhamnosus HA-114 was exclusive to TDP-43 or FUS pathogenesis or could be extended to other models of neurodegenerative diseases, we tested various models of ageassociated neurodegeneration, including models of Huntington's disease. We found that that $L$. rhamnosus HA-114 was also able to rescue paralysis phenotypes in worms expressing pan-neuronal polyglutamine repeats (Q40 and Q67, disease alleles) (Gidalevitz et al., 2006) (Figure 1F-G). These data suggest that paralysis and neurodegeneration phenotypes can be modulated through dietary probiotic intervention in nematodes and that $L$. rhamnosus HA-114 is effective in rescuing these phenotypes in several age-dependent models of neurodegeneration.

\section{Classic metabolic and stress pathways in C. elegans are not implicated in HA-114-mediated neuroprotection}

Next, we sought to investigate the potential mechanisms underlying the neuroprotective effect of $L$. rhamnosus HA-114 by determining whether HA-114 required well-known and characterized pathways for exerting its neuroprotective effect. Using strains constructed with loss or partial loss-of-function mutations in worms with transgenic expression of the TDP-43 ${ }^{A 315 T}$ mutant proteins, we disrupted fundamental metabolic, stress and signaling pathways in C. elegans to assess their role in mediating the neuroprotective effects of $L$. rhamnosus HA-114. We found that HA-114 remained able to rescue the paralysis phenotype in TDP$43^{\mathrm{A} 315 T}$ animals independently of daf-16/FOXO, hsf-1/HSF1, sir-2.1/SIRT1 and aak-2/AMPk (Figure 2A). These results suggest that neither the insulin/IGF-1mediated signaling pathway, the heat-shock response, the sirtuin pathway nor the AMP-activated protein kinase signaling pathway are required for the 
neuroprotection provided by HA-114. Similar results were obtained in our FUS $\$ 57 \Delta$ worms (Figure S2).

Protein aggregation is considered as a major contributor to the etiology of neurodegenerative disorders and a hallmark of many late onset neurodegenerative diseases. Hence, we used transgenic $C$. elegans strains expressing YFP-tagged polyglutamine repeats (Q40, disease allele) under the unc-54 promoter to assess the role of HA-114 on aggregation. These animals show progressive formation of Q40::YFP foci as they age (Morley et al., 2002). HA-114 did not significantly decrease aggregation in L4 larvae, nor in day 1 adult worms when compared to worms fed with control OP50 bacteria (Figure 2B). These results suggest that the effect mediated by HA-114 on disease-associated phenotypes occurs through an alternative pathway independent of aggregation. Subsequently, we screened several GFP reporter strains associated with genes implicated in major conserved stress response pathways. Transgenic reporter strains were fed with OP50 or HA-114 and fluorescence was compared at day 1 of adulthood. Compared to worms fed with OP50, HA-114-fed worms showed no difference in GFP expression for the following reporters: nlp-29::GFP, hsp-6::GFP, $d v e-1:: G F P$ and $h s p-4:: G F P$ (Figure 2C). These results likely exclude the innate immune response $(n / p-29)$, the mitochondrial unfolded protein response (UPR ${ }^{\text {mito; }}$ $h s p-6$ and $d v e-1)$ and the endoplasmic reticulum unfolded protein response (UPR ${ }^{\mathrm{ER}}$; $h s p-4$ ) as key pathways activated by L. rhamnosus HA-114. Altogether, these results suggest that $L$. rhamnosus HA-114 neuroprotection may be independent of classic longevity and stress response pathways studied in $C$. elegans.

\section{Fatty acid metabolism genes are essential for HA-114-mediated neuroprotection}

In order to gain insights on which category of genes might be implicated in HA-114 protective effect, we conducted whole-worm coding RNA sequencing (RNA-seq) 
of N2 worms fed with different bacterial strains. We compared worms fed with $L$. rhamnosus HA-114 to those fed with OP50, considered as the standard laboratory food source of $C$. elegans, or Bifidobacterium animalis subsp. lactis B94, a strain with no effect on paralysis nor neuronal health in our models of age-dependent neurodegeneration. RNA-seq results showed that the vast majority of differentially expressed genes were upregulated, with more than 300 genes having a fold change higher than 3. Enrichment gene ontology analysis showed that several categories of genes were upregulated exclusively in HA-114 samples, revealing lipid metabolism and oxidation-reduction as ones of the top categories (Figure 3A). Many genes classified in the category oxidation-reduction are also involved in lipid metabolism processes. These results are consistent with recent studies suggesting that gut microbiome can influence lipid metabolism in various systems (Matey-Hernandez et al., 2018). Moreover, lipid metabolism may play a role in both protection and deterioration of neurons in various neurodegenerative disorders (Dupuis et al., 2008; Shamim et al., 2018).

Next, we investigated the potential role of lipid metabolism and oxidationreduction biological processes by screening various transgenic GFP reporter strains. We quantified GFP fluorescence intensity of day 1 adult worms fed with OP50 or L. rhamnosus HA-114. Of all GFP reporters tested, only transgenic acdh$1:: G F P$ reporter strain displayed a significant change in fluorescence when fed with HA-114, although acdh-1::GFP worms displayed high GFP intensity when fed with control OP50 bacteria (Figure 3B). The acdh-1 gene is the orthologue of human $A C A D S B$ and a member of the ACDH family, which has oxidoreductase activity and is widely involved in fatty acid $\beta$-oxidation (Watts and Ristow, 2017). To confirm the role of acdh-1 in HA-114-mediated neuroprotection, we generated a strain harboring the transgenic FUS allele with an acdh-1 null background (FUS ${ }^{557}$; acdh-1(ok1489)). We observed no significant difference in paralysis rate over 12 days in these animals fed with either OP50 or HA-114 (Figure 3C). However, we were unable to generate a similar strain using TDP-43 ${ }^{\text {3 } 15 T}$ 
transgene. These results suggest that acdh-1 is a mechanistically essential gene for the neuroprotective effect provided by L. rhamnosus HA-114.

Extending our analysis to other genes involved in fatty acid metabolism, we hypothesized that kat-1, which is an orthologue of human ACAT1 and also involved in fatty acid $\beta$-oxidation, and elo-6, an orthologue of human ELOVL3 and ELOVL6 coding for a fatty acid elongase, might be involved in the paralysis rescuing effect of HA-114. Hence, we generated FUS ${ }^{S 57 \Delta}$; kat-1(tm1037) and the FUS ${ }^{S 57 \Delta}$; elo6(gk233) strains, harboring a loss-of-function mutation in kat-1 and elo-6. Feeding these mutant strains with HA-114 did not rescue paralysis phenotypes, suggesting a role for kat-1 and elo-6 in the neuroprotective effect of HA-114 (Figure 3D-E). HA-114 also did not rescue paralysis in both TDP-43A315T; kat-1(tm1037) and TDP43 ${ }^{\mathrm{A} 315 \mathrm{~T}}$; elo-6(gk233) strains, and even increased paralysis in a elo-6 loss-offunction background (Figure S3). These three genes (acdh-1, kat-1, elo-6) share a common metabolic pathway, involving fatty acids elongation and degradation in order to provide energy to the cell (Figure S4). Collectively, these results suggest that fatty acid metabolism, and more specifically $\beta$-oxidation, is a key component in the mechanism underlying the neuroprotective effect of HA-114 in agedependent neurodegeneration models.

\section{L. rhamnosus HA-114 fatty acids are essential for improving neurodegenerative phenotypes}

In order to better understand how HA-114 exerted its neuroprotective effect, we sought to investigate which component of $L$. rhamnosus HA-114 contributed to these beneficial effects. Recently, differential effects between live and heat-killed probiotics on general health have been observed (Sang et al., 2014; Sugahara et al., 2017). To investigate the importance of HA-114 viability for its rescue effect on the disease phenotype, we fed the FUS ${ }^{557 \Delta}$ worms with live or heat-killed bacteria from L1 stage to day 12 of adulthood. We observed a similar protective effect against paralysis in animals fed with either heat-killed or live HA-114 compared 
with animals fed with OP50 (Figure 4A). In order to identify which component of HA-114 was responsible for its neuroprotective activity, we assessed whether protein/amino acid or fatty acid (FA) extracts from HA-114, mixed with standard OP50 and used as a food source, were sufficient to provide beneficial effects against the age-dependent paralysis phenotype in the ALS model strain FUS ${ }^{557 \Delta}$ worms. While FUS ${ }^{557 \Delta}$ worms fed with $500 \mu \mathrm{g} / \mathrm{ml}$ protein extract from either OP50 or HA-114 displayed a similar paralysis phenotype (Figure 4B), those fed with 400 $\mathrm{nM}$ of FA extract from HA-114 showed a reduction of the phenotype compared to controls (Figure 4C). This fatty acid extract contains a mix of FA from HA-114, including medium-chain fatty acids (MCFAs, 6-12 carbons long), long-chain fatty acids (LCFAs, 13-21 carbons) and very-long chain fatty acids (vLCFAs, 22 carbons and more).

Contrary to short or medium-chain fatty acids which can freely enter the mitochondria to be oxidized, long-chain fatty acids need to be actively transported into the mitochondria in order to be part of the $\beta$-oxidation process. The classical transporter for LCFAs is the carnitine-shuttle, a conserved protein complex located on the mitochondrial membrane (Watts and Ristow, 2017). Specifically, carnitine palmitoyltransferase-1 (cpt-1/CPT1) is an important component of the carnitine shuttle complex at the outer face of the mitochondrial membrane. To assess the role of the carnitine shuttle on the motor phenotype of FUS ${ }^{S 57 \Delta}$ animals, we used etomoxir, a specific CPT-1 inhibitor which prevents LCFA transport into the mitochondria by blocking the formation of acyl carnitine, a key component in the carnitine-shuttle machinery. We hypothesized that if LCFAs were mediating the rescue effect provided by HA-114, inhibiting LCFA transport should amplify the neurodegeneration phenotype in the FUS ${ }^{557 \Delta}$ C. elegans model by impairing proper $\beta$-oxidation and altering energy homeostasis. As expected, we observed that blocking cpt-1/CPT1 was sufficient to significantly increase paralysis phenotypes in our model (Figure 4D). Furthermore, treating OP50- or HA-114-fed FUS ${ }^{557 \Delta}$ worms with $40 \mu \mathrm{M}$ of Etomoxir revealed that blocking cpt-1 was not sufficient to prevent the HA-114 bacterial strain from rescuing the paralysis 
phenotype (Figure 4E). Collectively, these results support the notion that impaired $\beta$-oxidation can worsen motor phenotypes and that HA-114-derived fatty acids can bypass the carnitine-shuttle to enter the mitochondria to be processed by the $\beta$ oxidation chain. This finding is consistent with evidence of an alternative pathway implicating other transporters for LCFAs, FAT/CD36 and SLC27 (Anderson and Stahl, 2013; Campbell et al., 2004; Glatz and Luiken, 2017).

As a proof-of-concept experiment, we then assessed cpt-1 expression levels in FUS ${ }^{S 57 \Delta}$ and FUS ${ }^{W T}$ transgenic animals. As expected, qRT-PCR using TAQMan probes of $c p t-1$ revealed that mutant FUS expressed less $c p t-1$ transcripts when normalized to ama-1 mRNA level and compared to control animals (Figure 4F). CPT1A mRNA expression is also decreased in liver of $\mathrm{hSOD} 1^{\mathrm{G} 93 \mathrm{~A}}$ transgenic mice, when compared to non-transgenic (Non-tg) littermates (Figure 4G). Finally, bioinformatics analysis of brain transcriptome from Prudencio et al. dataset revealed that expression of two transcripts related to carnitine palmitoyltransferase (CHKB_CPT1B and CPT1C) is decreased in cerebellum, but not in frontal cortex, of C9orf72 patients (Prudencio et al., 2015) (Figure 4H). Expression of other genes implicated in $\beta$-oxidation and lipid metabolism is also differentially expressed in C9orf72 patients cerebellum and frontal cortex (Figure S5). Taken together, these results suggest that fatty acids are the active component of HA114 bacterial strain and they can bypass the carnitine shuttle machinery. Moreover, these results point toward intrinsic carnitine shuttle issues in ALS pathogenesis.

\section{L. rhamnosus HA-114 restores lipid homeostasis in age-related neurodegeneration models}

Over the last few years, many studies have implicated impaired lipid metabolism in various neurodegenerative disorders, including Alzheimer's disease and ALS (Abdel-Khalik et al., 2017; Shamim et al., 2018; Wong et al., 2017). Since our data suggested that $\mathrm{HA}-114$ requires the fatty acid metabolism key component kat-1 
and increases acdh-1 expression, we hypothesized that the age-related neurodegeneration models we used might have disrupted lipid equilibrium and that HA-114 might restore lipid content to normal levels. We stained day 1 adult worms with Oil-Red-O dye to visualize neutral lipids. We observed higher content of lipid droplets in animals expressing TDP-43 ${ }^{\mathrm{A3} 15 \mathrm{~T}}$, FUS ${ }^{557 \Delta}$, Q40 or Q67 transgenes when compared to N2 animals (Figure 5A-B). Interestingly, this lipid accumulation was restored to control levels when animals were fed with HA-114 instead of OP50. Our data demonstrate that impaired lipid homeostasis is a feature of various models of neurodegenerative disorder and that HA-114 can rescue this phenotype. Lipid accumulation is often associated with impaired $\beta$-oxidation (Fromenty et al., 2004), which is consistent with what we have observed in our models.

\section{Impaired $\beta$-oxidation shortens lifespan and exacerbates neurodegeneration}

As previously mentioned, our findings are far consistent with evidence of an alternative pathway implicating other transporters for LCFAs, including FAT/CD36 and SLC27 family members. To further investigate the potential implication of this pathway in disease phenotype and neurodegeneration, we generated a strain harboring the transgenic FUS allele with an acs-20 null background, the worm ortholog of SCL27A. Since acs-20(tm3278) mutants display impaired locomotion at a young age, we were unable to study paralysis phenotype on this strain nor in FUS ${ }^{574}$;acs-20(tm3278) worms. In order to study other phenotypes, we evaluated

lifespan of these animals. We hypothesized that acs-20 mutants might negatively regulate longevity and that our mutant FUS animals might be sensitive to this lossof-function. Interestingly, while FUS ${ }^{S 57 \Delta}$ transgenics do have lifespan similar to N2, acs-20(tm3278) exhibited shortened lifespan that was exacerbated in our FUS ${ }^{5574}$;acs-20(tm3278) animals (Figure 6A). To assess the effects of complete inhibition of LCFAs transport, we evaluated lifespan of our worms using Etomoxir, a specific inhibitor of $c p t-1 / C P T 1$. We observed that blocking both $c p t-1 / C P T 1$ and acs-20/SLC27A was sufficient to significantly decrease lifespan in our FUS ${ }^{557 \Delta}$ when compared to N2. Moreover, acs-20(tm3278) mutant had shorter lifespan. 
This effect was also additive in our FUS ${ }^{S 574}$;acs-20(tm3278) animals, which display the shorter lifespan of all conditions (Figure 6B). Next, we assessed whether impairing $\beta$-oxidation using acs-20(tm3278) or acdh-1(ok1489) mutants can contribute to neurodegeneration. While N2 and mutant FUS display similar low rates of neurodegeneration, both acs-20 and acdh-1 mutants were associated with GABAergic motor neuron loss as early as day 1 . We also found a significant increase in motor neuron degeneration levels in both FUS 5574 ;acs-20(tm3278) and FUS ${ }^{574}$;acdh-1(ok1489) animals (Figure 6C). Taken together, these results suggest that acdh-1/ACADSB and acs-20/SLC27A can influence lifespan, and that impaired $\beta$-oxidation might play an important role in neurodegeneration.

\section{L. rhamnosus HA-114 delays disease onset and prevents neurodegeneration in SOD1 ${ }^{\text {G93A }}$ mouse model}

In order to test if our probiotic-mediated neuroprotection was also efficient in a more complex system, we treated SOD1 ${ }^{\mathrm{G} 93 \mathrm{~A}}$ mice, a well-characterized mouse model of ALS (Gurney et al., 1994), with L. rhamnosus HA-114, from P70 until they reached end stage. Mice were monitored every 5 days until disease onset, then they were monitored three times a week. Dietary HA-114 intervention did not extend lifespan in our model (Figure S6A). However, treatment with HA-114 probiotic was sufficient to significantly delay onset of the disease (Vehicle: $113.6 \pm 1.484$ days, $N=20$; HA-114 : $118.8 \pm 1.516$ days; $N=20$ ), as well as decreasing disease duration (Vehicle : $23.6 \pm 2.688$ days, $N=20$; HA-114 : 17.5 \pm 1.428 days, $N=20$ ) (Figure 7). HA-114 dietary supplementation had no effect on body weight, hangwire tests, or neurological scores (Figure S6B-D). We then wanted to investigate if HA-114 was able to act directly on motor neuron health and prevent neurodegeneration. We used the general neuronal nuclei marker NeuN as well as the cholinergic neuron marker ChAT to specifically identify motor neurons (MNs) in lumbar spinal cords cross sections. Neurons in the ventral horn of the spinal cord positive for both markers were counted as a-MN. Analysis revealed that HA-114 treatment was able to partially rescue MNs 
neurodegeneration in symptomatic SOD $1^{\mathrm{G} 93 \mathrm{~A}}$ animals. Data were normalized to SOD1 ${ }^{\mathrm{WT}}$ values. (Figure 7C; SOD1 ${ }^{\mathrm{WT}}$ : $100 \pm 10.47 \%, N=5$; Non-Tg: $97.63 \pm 4.94 \%$, $N=5$; SOD1 ${ }^{\mathrm{G} 93 \mathrm{~A}}$ with vehicle: $45.98 \pm 5.68 \%, N=5$; SOD1 ${ }^{\mathrm{G} 93 \mathrm{~A}}$ with HA-114: 73.78. $\pm 5.58 \%, N=5)$. Taken together, our results show that microbiome modulation through probiotics supplementation can improve healthspan in SOD1 ${ }^{\text {G93A }}$ mice model of ALS by delaying disease onset, decreasing disease duration and preserve MNs from neurodegeneration.

\section{Discussion}

Microbiome research has highlighted the importance of the gut-brain axis in human health. Gut flora has primarily been studied in cases of inflammatory diseases, but emerging data is beginning to link microbiome components to neurodegenerative disorders, including Parkinson's and Alzheimer's diseases. Although some bacterial strains have been suggested to play a role in neurodegeneration, so far no strain has been formally associated with this process (Astafurov et al., 2014; Dopkins et al., 2018; Mulak and Bonaz, 2015; Vogt et al., 2017; Wu et al., 2015).

We investigated the potential beneficial role of microbiome in neurodegenerative diseases and discovered that a probiotic strain, Lacticaseibacillus rhamnosus HA114 , was able to positively modulate disease phenotype in multiple $C$. elegans models of age-dependent neurodegeneration. Our results demonstrate the ability of a bacterial strain to restore multiple motor or neurodegeneration phenotypes. HA-114 is the only L. rhamnosus strain tested showing neuroprotective effect. Moreover, we excluded the contribution of classic metabolic and stress responses pathways into the beneficial effect provided by HA-114. We also identified acdh$1 / A C A D S B$, kat-1/ACAT1 and elo-6/ELOVL3/6 as key components of the neuroprotection provided by the HA-114 strain. Two of those genes are involved in two distinct metabolic pathways: fatty acid metabolism, more precisely mitochondrial $\beta$-oxidation, and branch-chained amino acid breakdown (BCAA). Our results show that providing protein extract from HA-114, was not sufficient to recapitulate the beneficial effect seen with HA-114. Interestingly, HA-114-derived 
fatty acids were sufficient to rescue motor phenotype in our C. elegans ALS model. These results suggest that $\beta$-oxidation is the favored pathway in this context. Mitochondrial fatty acid $\beta$-oxidation disorders are associated with many symptoms including neuropathy (Vishwanath, 2016). Moreover, we identified lipid homeostasis dysregulation in various $C$. elegans models of age-dependent neurodegeneration. Augmentation of lipid droplets is often associated with improper $\beta$-oxidation, reflecting the incapacity of mitochondria to oxidized fatty acids and use them as an energy source (Lee et al., 2013). Finally, we identified acs-20/SLC27A, an alternative entry point for LCFAs into the mitochondria, as an essential modulator of lifespan in our $C$. elegans ALS model as well as an important contributor to neurodegeneration.

An important feature of the vast majority of age-related neurodegenerative disorders is the accumulation and aggregation of misfolded proteins in the cytosol suggesting potential common pathogenic mechanisms. We demonstrated that $L$. rhamnosus HA-114 was not able to prevent aggregation, while still being effective to protect motor neurons from degeneration. Neurodegeneration may in part be caused by metabolic and energy imbalances associated with the expression of mutant genes in aging motor neurons. Interventions that can compensate for the loss of energy production may bolster the cells ability to restore lipid homeostasis and energy production, ultimately delaying or halting neurodegeneration. Our results suggest that dietary supplementation with $L$. rhamnosus HA-114 provides key nutrients driving energy production, helping to mitigate metabolic dysfunction leading to neurodegeneration (Figure 8).

Disrupted energy homeostasis is well studied in ALS, as well as in other neurodegenerative disorders (Vercruysse et al., 2018). The majority of ALS patients present hypermetabolism, hyperlipidemia as well as insulin resistance (Ahmed et al., 2016). Interestingly, ALS patients with high body mass index (BMI) have better prognosis than patient with BMI within the normal values. Moreover, obese individuals are less likely to develop ALS than individuals with average 
weight (Jawaid et al., 2018). However, BMI does not differentiate between fat or lean body mass. Furthermore, ALS patients display non-alcoholic fatty liver disease, without being overweight or obese, suggesting lipid homeostasis impairment (Dupuis et al., 2008; Nodera et al., 2015). Similar lipid disequilibrium phenotypes can be observed in transgenic mouse models of ALS (Lee and Yang, 2018) and Alzheimer's disease (Kim et al., 2016). Hepatic steatosis (fatty liver degeneration) seems to be a common phenotype associated with neurodegenerative disorders and neurodegeneration caused by viral infections (Gupta et al., 2012; Nash et al., 2016; Zolkipli et al., 2012). A common clinical observation in ALS patients is weight loss. Although fat accumulation is a phenotype we see in C. elegans models, it is not possible to directly extrapolate this phenomenon to ALS patients. However, it is a marker of impaired lipid homeostasis. Even if $C$. elegans are devoid of specialized adipose tissue or liver, many of the genes required for lipid metabolism are highly conserved.

We demonstrated that the carnitine shuttle is impaired in animals expressing mutant ALS genes, with reduced mRNA expression of $c p t-1 / C P T 1$. ALS patients are known to have lower L-carnitine levels than healthy subjects (Lawton et al., $2014 ; 2012$ ) and L-carnitine is essential for $\beta$-oxidation via the carnitine shuttle, where CPT1/2 are required. This shuttle is typically used for long or very longchain fatty acids, while short and medium chain fatty acids can enter the mitochondria independently. However, if ALS patients have lower L-carnitine, it means they have decreased $\beta$-oxidation. Our data suggest that the real problem may not be the low L-carnitine levels by itself, but that ALS patients/models have less active CPT1/2 receptors. This could also explain why ALS patients have higher long-chain fatty acids in their cytosolic triacylglycerol pools (Blasco et al., 2017), and these molecules may contribute to loss of lipid homeostasis if not properly metabolized in the mitochondria.

Interestingly, L-carnitine treatment was protective in a mouse model of ALS (Kira et al., 2006), and promising endpoints were observed in a small ALS clinical trial for patients treated with acetyl-L-carnitine (Beghi et al., 2013). Data from 
Drosophila ALS models points toward dysfunction of the carnitine shuttle as a potential mechanism (Manzo et al., 2018). Altogether, these studies suggest that impaired $\beta$-oxidation, and perhaps the carnitine shuttle contributes to motor neuron degeneration in ALS. Since misfolded proteins can interact with mitochondria (Pasinelli et al., 2004; Ruan et al., 2017; Vande Velde et al., 2008), they may directly interact with carnitine shuttle proteins, or alter mitochondrial morphology leading to impairment of the shuttle. Our results also demonstrate that loss-offunction of both acs-20/SLC27A and acdh-1/ACADSB have the capacity to induce motor neuron loss in a model prone to neurodegeneration. This study is the first effective demonstration of gut microbiome modulation through dietary intervention of probiotics using several ALS models, as well as the first to demonstrate a link between impaired $\beta$-oxidation and neurodegeneration.

Our results also demonstrate that HA-114 dietary supplementation was sufficient to delay disease onset, decrease disease duration and prevent neurodegeneration in an ALS mammalian model, the SOD1 ${ }^{\text {G93A }}$ mouse, while having no significant effect on other parameters, including lifespan. This model displays a very aggressive phenotype, with a short treatment window available according to guidelines (Ludolph et al., 2010). Modulation of microbiome is a chronic treatment and might take longer to be fully effective on several aspects of health span. The lack of lifespan extension is however consistent with our results in C. elegans, emphasizing the translational aspect of our work and the consistency of our results. Additional studies in mammalian models displaying milder phenotypes are essential to characterize the beneficial scope of Lacticaseibacillus rhamnosus HA114. This study is the first effective demonstration of gut microbiome modulation through dietary intervention of probiotics using several ALS models.

Moreover, improving health span and disease management is of growing interest in various neurodegenerative disorders, including ALS, where drugs have repeatedly failed to cure these diseases. The only approved drugs for ALS are Riluzole and Radicava (Edaravone), which have modest effects on survival or symptoms (Abe et al., 2014; Miller et al., 2012; Nagase et al., 2016; Takahashi et 
al., 2017). Moreover, Riluzole recently failed to prolong survival in SOD $1^{\mathrm{G} 93 \mathrm{~A}}$ and other mammalian models, while still being widely used to treat ALS, meaning that lifespan extension might not be the main parameter to consider to move forward clinical investigation (Hogg et al., 2018). With the revolution in gene discovery for ALS, and the prohibitively long time needed to develop accurate mouse models for each new gene discovered, simpler animal models are needed to bridge the gap and help guide the development of higher models. Our investigation of bacterial strain-specific neuroprotection provides new insights into neurodegeneration by identifying impaired lipid homeostasis as a feature of the disease, while also identifying new potential therapeutic strategies. Importantly, severe side effects are a major reason for discontinuation of clinical drug development (frequently in phase III). Thus, probiotics may be an alternative or complementary approach for neurodegenerative diseases since chronic treatment with probiotics is associated with a low risk of side effects. 


\section{Material and methods}

\section{C. elegans maintenance and strains}

C. elegans were maintained as previously described (Stiernagle, 2006). Briefly, worms were kept on NGM agar plates that were streaked with E. coli OP50, Lacticaseibacillus rhamnosus HA-114 or other probiotics as food source (Table 1). All the probiotics strains were kindly provided by Lallemand Health Solutions (Montreal, Canada). All assays were performed at $20^{\circ} \mathrm{C}$. The N2 Bristol strain, as well as EG1285 (ox/s12 [unc-47p::GFP + lin-15(+)]), VS24 (kat-1(tm1037)), VC1011 (acdh-1(ok1489)), VL749 (wwls24[Pacdh-1::GFP; unc-119(+)]), RB842 (abt-2(ok669)), RB817 (abt-4(ok633)), RB754 (aak-2(ok524)), CF1038 (daf16(mu86)), VC199 (sir-2.1(ok434)), PS3551 (hsf-1(sy441)), SJ4197 (zcls39[dve1p::dve-1::GFP]) , IG274 (frls7[nlp-29p::GFP,col-12p::DsRed]), SJ4100 (zcls13[hsp-6::GFP]), SJ4005 (zcls4([hsp-4::GFP; lin-15(n765), AM44 (rm/s190 [F25B3.3p::Q67::CFP]), AM101 (rm/s110 [F25B3.3p::Q40::YFP]) and AM141 (rm/s133 [unc-54p::Q40::YFP]) were obtained from the C. elegans Genetics Center (University of Minnesota, Minneapolis). FX03278 (acs-20(tm3278)) was obtained from S. Mitani and the Japanese National BioResource Project (Tokyo, Japan). Mutant strains were outcrossed to N2 4 times before use. Other $C$. elegans strains were obtained by crossing. Homozygosity of all genotypes was confirmed by PCR or sequencing.

Transgenic lines expressing mutant TDP-43 ${ }^{\mathrm{A} 315 \mathrm{~T}}$, wild-type TDP-43 (TDP-43 ${ }^{\mathrm{WT}}$ ), mutant FUS ${ }^{557 \Delta}$ and wild-type FUS (FUS ${ }^{\mathrm{WT}}$ ) were created as previously described (Vaccaro et al., 2012). Several strains showing comparable phenotypes and transgene expression levels were kept and the strains used in this study include :

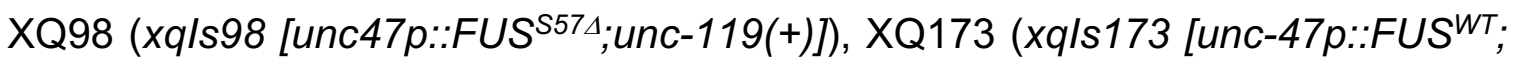
unc-119(+)]), XQ132 (xq/s132 [unc-47p::TDP-43WT; unc-119(+)]) and XQ133 (xqls133 [unc-47p::TDP-43 ${ }^{A 315 T}$; unc-119(+)]). 


\section{Paralysis assay on solid media}

Briefly, 40 age-synchronized L4 worms were transferred to NGM plates and scored daily for paralysis, from day 1 to day 12 of adulthood. Animals were counted as paralyzed if they failed to move upon prodding with a worm pick. Worms were scored as dead if they failed to move their head after being prodded on the nose and showed no pharyngeal pumping. All experiments were conducted at $20^{\circ} \mathrm{C}$ and in triplicates, three times. Some experiments were conducted by dissolving Etomoxir (40 $\mu \mathrm{M}$, Medchem express) or Paraquat (250 $\mu \mathrm{M}$, Sigma-Aldrich) into the NGM plates.

\section{Lifespan assay}

Approximately 40 age-synchronized L4 worms were transferred NGM plates streaked with OP50 or HA-114 and tested every 2 days from day 1 adult until death. Worms were scored as dead if they failed to respond to tactile stimulus and showed no spontaneous movement or response when prodded. Dead worms displaying internally hatched progeny or extruded gonads or worms that crawled off the plate were excluded. All experiments were conducted at $20^{\circ} \mathrm{C}$ and in triplicates. Some experiments were conducted by dissolving Etomoxir (Medchem express) into the NGM plates at a concentration of $40 \mu \mathrm{M}$.

\section{Neurodegeneration assay}

For scoring of neuronal processes for gaps or breakage, worms were selected at day 9 of adulthood for visualization of motor neuron in vivo. Animals were immobilized in $5 \mathrm{mM}$ levamisole dissolved in $\mathrm{M} 9$ and mounted on slides with $2 \%$ agarose pads. GFP was visualized at $505 \mathrm{~nm}$ using a Zeiss Axio Imager M2 microscope, using a 20X objective and a 1.5 Optovar. The software used was AxioVs40 4.8.2.0. At least one hundred worms were scored per condition, over 4 distinct experiments. 


\section{Aggregation assay}

Briefly, worms were synchronized and fed with OP50 or Lacticaseibacillus rhamnosus HA-114 until they reach L4 stage or day 1 adult. Visible aggregates were scored in each worm and animals were divided into three categories of aggregation: light (less than 5), moderate (between 5 and 15) and strong (more than 15). Between 215 and 240 worms per condition were tested over 3 trials.

\section{C. elegans fluorescence microscopy}

For visualization of GFP worms, a $5 \mathrm{mM}$ solution of levamisole diluted in M9 was used for immobilization. Animals were mounted on slides with $2 \%$ agarose pads. Fluorescent expression for quantification was visualized with a Zeiss microscope AxioObserver Z1. The software used was AxioVs40 4.8.2.0. Thirty day-1 adult worms were visualized per condition, over three different experiments. Image processing and quantification were done with Fiji. To compare fluorescence, we calculated the changes in the ratio (size/intensity of fluorescence).

\section{Oil Red $O$ staining, imaging and quantification}

Briefly, Oil Red $\mathrm{O}$ stock solution was made at a concentration of $10 \mathrm{mM}$ and balanced over two days. Working solution was freshly made before each use at a concentration of $6 \mathrm{mM}$ and filtered. Age-synchronized day 1 adults worms were fixed in PBS/isopropanol 60\%/0,01\% Triton-X for 15 min at RT and then stained overnight at RT with Oil red $O$ working solution. Worms were washed 3 times with PBS $/ 0,01 \%$ Triton- $X$ and mounted on slides with $2 \%$ agarose pads. Oil red $O$ staining was visualized using an Olympus brightfield microscope with a DP21 microscope digital system and a 10x objective. Images were quantified using Fiji (ImageJ) software. ORO stain was quantified in 56-111 animals per condition, over 3 different sets of experiments. 


\section{Gene expression and RNA-Seq analysis}

Total RNA was obtained from synchronized adult day $1 \mathrm{C}$. elegans and liver from symptomatic (P120) mice using the "RNA extraction for RNA-Seq" protocol from the Bowdish Lab (McMaster University, Hamilton, Canada), quantified photometrically with a NanoPhotometer (Implen) and stored at $-80^{\circ} \mathrm{C}$ until further use. For gene expression analysis, cDNA from $800 \mathrm{ng}$ total RNA was generated using the Superscript Vilo cDNA synthesis kit (Thermo Fischer Scientific). Samples were used, undiluted, and yielded a CT value between 15 and 25. Gene expression was analyzed using TaqMan Gene Expression Assays (Applied Biosystems) and a QuantStudio 3 Real-Time PCR System (Thermo Fisher). Data were normalized to the housekeeping gene ama-1 (for C.elegans) or Polr2a (for mice) and analyzed using the $\Delta / \Delta-C T$ method. All experiments were made in triplicates, three times. For RNA-Seq, library preparation and sequencing was made at the Institute for Research in Immunology and Cancer (IRIC) Genomics Platform and analyzed by the bioinformatics service (Université de Montréal). 500 ng of total RNA was used for library preparation. Quality of total RNA was assessed with the BioAnalyzer Nano (Agilent) and all samples had a RIN above 7. Library preparation was done with the KAPA mRNAseq hyperprep standed kit (KAPA, Cat no. KK8421). Ligation was made with $1.4 \mathrm{nM}$ final concentration of Illumina Truseq index and 16 PCR cycles was required to amplify cDNA libraries. Libraries were quantified by QuBit and average library length was evaluated with the BioAnalyzer DNA1000. All libraries were diluted to $10 \mathrm{nM}$ and normalized by qPCR using the KAPA library quantification kit (KAPA; Cat no. KK4973). Libraries were pooled to equimolar concentration. Sequencing will be performed with the Illumina Nextseq500 using the Nextseq High Output 75 cycles (1x75bp) using 2 pM of the pooled library. Around $25 \mathrm{M}$ single-end reads was generated per sample. Sequences were trimmed for sequencing adapters and low quality 3 ' bases using Trimmomatic version 0.35 (Bolger et al., 2014) and aligned to the reference C.elegans genome version WBcel235 (gene annotation from Ensembl version 84) using STAR version 2.5.1b (Dobin et al., 2012). Gene expressions were obtained both as readcount directly from STAR as well as computed using RSEM(Li and 
Dewey, 2011) in order to obtain gene and transcript level expression, either in TPM or FPKM values. DESeq2 version 1.6.2 (Love et al., 2014) was then used to identify differentially expressed genes. Sample clustering based on normalized log read counts produces the following hierarchy of samples. To identify significant biological pathways in which differentially expressed genes were enriched, geneontology (GO) term analysis was conducted using PANTHER 11 (Ashburner et al., 2000; Mi et al., 2017; The Gene Ontology Consortium, 2019).

\section{Bioinformatics analysis}

The RNA-sequencing data from human frontal cortex and cerebellum from Prudencio et al. dataset (Prudencio et al., 2015) (GEO accession: GSM1642314; SRA study:SRP056477) a were aligned using HiSAT2 against reference genome Hg38. Read counts were obtained HTSeq-count and differential expression analysis were performed with the Bioconductor R package DESeq2.

\section{Fatty acids extraction and measurements}

A fatty acid extraction kit (MAK174, Sigma) was used to perform the extraction. Pellets of OP50 and L. rhamnosus HA-114 were homogenized in the provided extraction buffer and vortexed. The extraction was done as described in the provided protocol. All fatty acids extractions were freshly made on the day of their use. Samples were quantified using colorimetric assay as described in the provided protocol of the Free fatty acids quantification kit used (MAK044-1KT, Sigma). Experiments were conducted by adding 400 nmoles of OP50 or $L$. rhamnosus HA-114 fatty acids extracts on OP50-streaked plates.

\section{Protein extraction and measurements}

OP50 and L. rhamnosus HA-114 pellets were lysed in RIPA buffer $(150 \mathrm{mM} \mathrm{NaCl}$, $50 \mathrm{mM}$ Tris $\mathrm{pH} 7.4,1 \%$ Triton $\mathrm{X}-100,0.1 \%$ SDS and $1 \%$ sodium deoxycholate) containing $0.1 \%$ protease inhibitors $(10 \mathrm{mg} / \mathrm{ml}$ leupeptin, $10 \mathrm{mg} / \mathrm{ml}$ pepstatin $A$ and $10 \mathrm{mg} / \mathrm{ml}$ chymostatin LPC; 1/1000). Pellets were passed through a 27.5-gauge syringe ten times, sonicated for $5 \mathrm{~min}$, and centrifuged at $16,000 \mathrm{~g}$ for $10 \mathrm{~min}$ at 
$4^{\circ} \mathrm{C}$. Supernatants were collected in $1.5 \mathrm{~mL}$ tubes. The supernatants were quantified using the BCA protein assay kit (Thermo Scientific) following the manufacturer's protocol and instructions. Samples were stored at $-80^{\circ} \mathrm{C}$ until their use. Experiments were conducted by adding $500 \mathrm{ug} / \mathrm{mL}$ of OP50 or L. rhamnosus HA-114 proteins extracts on OP50-streaked plates.

\section{Mouse breading and maintenance}

SOD1 ${ }^{\text {G93A }}$ transgenic mice have been previously described (Gurney et al., 1994). Male SOD $1^{\text {G93A }}$ progeny were used for further breeding to maintain the line. Only female mice were used for these experiments. Animals were treated according to our approved protocols from the CRCHUM Institutional Committee for the Protection of Animals and the Canadian Council on Animal Care (CCAC). For all experiments, mice were housed under controlled conditions, with a 12-h light/dark cycle and with food and water (or water with HA-114) given ad libitum.

\section{Experimental group and Lacticaseibacillus rhamnosus HA-114 treatment}

All procedures were carried out in accordance with the Guidelines for Preclinical Animal Research in ALS/MND (Ludolph et al., 2010). Transgenics littermates were separated into the two conditions, while making sure that each mouse in the HA114-treated group had a corresponding littermate in the control group. 20 mice per group were used. Mice received HA-114 or regular water (vehicle) by spontaneous oral administration ( $10^{9} \mathrm{CFU} / \mathrm{ml}$ per day), every day starting from day 70 . Bottles were changed 3 times a week. HA-114 was provided by Lallemand Health Solutions.

\section{Determination of disease onset and end-stage}

All mice were weighted, evaluated for motor activity (hangwire grip test) and neurological score every 5 days from day 70 , until we determined disease onset. After onset, mice were monitored 3 times a week. Neurological score was assessed using a 0-4 scale as developed by ALSTDI (ALS Therapy Development Institute) (Scott et al., 2008) (Hatzipetros et al., 2015) (Table 2). Disease onset 
was defined as the date when the mouse was first assessed with a neurological score of 1 . Once a score of 3 was reached, the mouse was closely monitored by the animal facility and the team and was evaluated every day until it reach score 4. Neurological score 4 was considered as our end-stage point and animals were euthanized as soon as they reach it.

\section{Hangwire assay}

Mice were placed on a wired housing cage lid. The lid was then gently inverted. The time from lid inversion until the mouse fall of the lid was measured. Each mouse was given up to three attempts a maximum of $90 \mathrm{~s}$. The mean of the three attempts were used for further analysis.

\section{Immunofluorescence of lumbar spinal cord sections}

Mice were anesthetized with isoflurane and then were perfused through the heart with cold Sorenson's buffer $1 \times$ for 5 min, followed by $4 \%$ formaldehyde diluted in PBS $1 \times(4 \%$ PFA) for $\sim 15 \mathrm{~min}$. The whole mouse was then further fixed overnight in $4 \%$ PFA at $4{ }^{\circ} \mathrm{C}$, and lumbar spinal cords were dissected, postfixed in $4 \%$ PFA overnight at $4^{\circ} \mathrm{C}$, and cryoprotected in a $30 \%$ sucrose PBS $1 \times$ solution for $72 \mathrm{~h}$ at $4^{\circ} \mathrm{C}$. Lumbar spinal cords were then embedded in OCT and snap frozen in cooled isopentane $\left(-40\right.$ to $\left.-50^{\circ} \mathrm{C}\right)$.

$14 \mu \mathrm{m}$-thick spinal cord cryosections were directly put on slide and washed twice (5 min each) in $0.3 \%$ Triton X-100 in PBS. Tissues were permeabilized with icecold methanol for $10 \mathrm{~min}$ at $-20^{\circ} \mathrm{C}$, washed 3 times in $0.3 \%$ Triton X-100 in PBS and then incubated in a blocking solution (3\% BSA, 0.3\% Triton X-100 in PBS) for $1 \mathrm{~h}$ at room temperature. Sections were incubated overnight with primary antibodies against choline acetyltransferase (ChAT; 1:75; goat, catalog \#AB144P, Millipore) and neuronal-specific nuclear protein (NeuN; 1:300; mouse IgG1, catalog \#MAB377, Millipore) in 1\% BSA, $0.3 \%$ Triton X-100 in PBS at $4^{\circ} \mathrm{C}$. Slices were then rinsed three times with PBS $1 \times(5$ min each) and incubated with the 
secondary antibody Alexa Fluor 594-donkey anti-goat (1:500; Jackson ImmunoResearch) in 1\% BSA, $0.3 \%$ Triton $\mathrm{X}-100$ for $2 \mathrm{~h}$ at room temperature. Slices were then rinsed three times with PBS $1 \times$ and incubated with Alexa Fluor 488-goat anti-mouse $\operatorname{lgG} 1$ secondary antibody for $1 \mathrm{~h}$. Finally, sections were rinsed three times with PBS $1 \times$ (5 min each) and mounted on Superfrost Plus Slides (Thermo Fisher Scientific) with Prolong Gold Antifade Reagent (Thermo Fisher Scientific).

Image acquisition was performed on a Zeiss microscope AxioObserver Z1 with a 20x objective (Plan-Apochromat 20x/0.8; Optovar 1). The software used was AxioVs40 4.8.2.0. Motor neurons from ventral horns were counted on at least 5 sections per animals, with 5 animals per condition. Results are expressed as the average number of cells counted per ventral horn in each animal, normalized to the values of SOD1 ${ }^{\mathrm{WT}}$ animals. All cell body counts and measurements were performed by an investigator who was blind to the identity of the tissue.

\section{Statistics}

For the $C$. elegans experiments, paralysis curves were generated and compared using the log-rank (Mantel-Cox) test. All experiments were repeated at least three times. For neurodegeneration and aggregation assay, one way ANOVA were performed. For fluorescence tests and gene expression analysis, unpaired t-test with Welch's correction were performed. For ORO quantification, two-way ANOVA was performed. For the SOD $1^{\mathrm{G} 93 \mathrm{~A}}$ mouse experiments, all curves were generated and compared using the log-rank (Mantel-Cox) test. Graph bars were compared using unpaired t-test with Welch's correction or Welch and Brown-Forsythe ANOVA, followed by Dunnett's T3 multiple comparisons test. Quantitative data were expressed as mean \pm SEM. GraphPad Prism v8 software was used for all statistical analyses, except the bioinformatics analysis, where $\mathrm{R}$ was used. 


\section{Author contributions}

A.L. contributed to the project design, performed experiments, analyzed and assembled the data and participate to manuscript writing. E.G helped with performing experiments and analyzing the data. G.T. performed the aggregation assay. E.B and M.L performed bioinformatics analysis. M.T supervised the bioinformatics analysis. J.A.P. directed the project and participated in manuscript writing.

\section{Acknowledgments}

We would like to thank Thomas A. Tompkins, Ph.D. of Lallemand Health Solutions inc, which provided probiotics strains, financial and technical support, the Caenorhabditis Genetics Center (CGC), S. Mitani and the Japanese National BioResource Project (NBRP), which provided many of the strains used in this study, E. Possik for the Oil Red O protocol and tips, J. Huber from the IRIC genomics platform for RNA-Seq as well as P. Gendron for RNA-Seq data analysis. A special thanks to S. Peyrard for technical support, C. Vande Velde for helpful discussions regarding mice experiments, $H$. Sidibé for the tips on TAQMan experiments, moral support and scientific thoughts, A. Cleret-Buhot and R. Manceau for their help with ORO quantification as well as J. Doyle and J-C Labbé for the helpful discussions. Figure 8 was created with BioRender.com. A.L. is supported by an ALS Canada trainee award and a FRQS trainee award. This project was funded by ALS Canada, Brain Canada and NSERC. 


\section{References}

Abdel-Khalik, J., Yutuc, E., Crick, P.J., Gustafsson, J.-Å., Warner, M., Roman, G., Talbot, K., Gray, E., Griffiths, W.J., Turner, M.R., et al. (2017). Defective cholesterol metabolism in amyotrophic lateral sclerosis. J. Lipid Res. 58, 267-278.

Abe, K., Itoyama, Y., Sobue, G., Tsuji, S., Aoki, M., Doyu, M., Hamada, C., Kondo, K., Yoneoka, T., Akimoto, M., et al. (2014). Confirmatory double-blind, parallelgroup, placebo-controlled study of efficacy and safety of edaravone (MCl-186) in amyotrophic lateral sclerosis patients. Amyotroph Lateral Scler Frontotemporal Degener 15, 610-617.

Ahmed, R.M., Irish, M., Piguet, O., Halliday, G.M., Ittner, L.M., Farooqi, S., Hodges, J.R., and Kiernan, M.C. (2016). Amyotrophic lateral sclerosis and frontotemporal dementia: distinct and overlapping changes in eating behaviour and metabolism. Lancet Neurol 15, 332-342.

Akbari, E., Asemi, Z., Daneshvar Kakhaki, R., Bahmani, F., Kouchaki, E., Tamtaji, O.R., Hamidi, G.A., and Salami, M. (2016). Effect of Probiotic Supplementation on Cognitive Function and Metabolic Status in Alzheimer's Disease: A Randomized, Double-Blind and Controlled Trial. Front Aging Neurosci 8, 256.

Anderson, C.M., and Stahl, A. (2013). SLC27 fatty acid transport proteins. Molecular Aspects of Medicine 34, 516-528.

Ashburner, M., Ball, C.A., Blake, J.A., Botstein, D., Butler, H., Cherry, J.M., Davis, A.P., Dolinski, K., Dwight, S.S., Eppig, J.T., et al. (2000). Gene ontology: tool for the unification of biology. The Gene Ontology Consortium. Nat. Genet. 25, 25-29.

Astafurov, K., Elhawy, E., Ren, L., Dong, C.Q., Igboin, C., Hyman, L., Griffen, A., Mittag, T., and Danias, J. (2014). Oral microbiome link to neurodegeneration in glaucoma. PLoS ONE 9, e104416.

Beghi, E., Pupillo, E., Bonito, V., Buzzi, P., Caponnetto, C., Chiò, A., Corbo, M., Giannini, F., Inghilleri, M., Bella, V.L., et al. (2013). Randomized double-blind placebo-controlled trial of acetyl-L-carnitine for ALS. Amyotroph Lateral Scler Frontotemporal Degener 14, 397-405.

Blacher, E., Bashiardes, S., Shapiro, H., Rothschild, D., Mor, U., Dori-Bachash, M., Kleimeyer, C., Moresi, C., Harnik, Y., Zur, M., et al. (2019). Potential roles of gut microbiome and metabolites in modulating ALS in mice. Nature 572, 474-480. 
Blasco, H., Veyrat-Durebex, C., Bocca, C., Patin, F., Vourc'h, P., Kouassi Nzoughet, J., Lenaers, G., Andres, C.R., Simard, G., Corcia, P., et al. (2017). Lipidomics Reveals Cerebrospinal-Fluid Signatures of ALS. Sci Rep 7, 1004.

Bolger, A.M., Lohse, M., and Usadel, B. (2014). Trimmomatic: a flexible trimmer for Illumina sequence data. Bioinformatics 30, 2114-2120.

Burberry, A., Wells, M.F., Limone, F., Couto, A., Smith, K.S., Keaney, J., Gillet, G., van Gastel, N., Wang, J.-Y., Pietilainen, O., et al. (2020). C9orf72 suppresses systemic and neural inflammation induced by gut bacteria. Nature $582,89-94$.

Campbell, S.E., Tandon, N.N., Woldegiorgis, G., Luiken, J.J.F.P., Glatz, J.F.C., and Bonen, A. (2004). A novel function for fatty acid translocase (FAT)/CD36: involvement in long chain fatty acid transfer into the mitochondria. Journal of Biological Chemistry 279, 36235-36241.

Clark, R.I., and Walker, D.W. (2017). Role of gut microbiota in aging-related health decline: insights from invertebrate models. Cellular and Molecular Life Sciences 75, 93-101.

Dobin, A., Davis, C.A., Schlesinger, F., Drenkow, J., Zaleski, C., Jha, S., Batut, P., Chaisson, M., and Gingeras, T.R. (2012). STAR: ultrafast universal RNA-seq aligner. Bioinformatics 29, 15-21.

Dopkins, N., Nagarkatti, P.S., and Nagarkatti, M. (2018). The role of gut microbiome and associated metabolome in the regulation of neuroinflammation in multiple sclerosis and its implications in attenuating chronic inflammation in other inflammatory and autoimmune disorders. Immunology 154, 178-185.

Dupuis, L., Corcia, P., Fergani, A., Gonzalez De Aguilar, J.-L., BonnefontRousselot, D., Bittar, R., Seilhean, D., Hauw, J.-J., Lacomblez, L., Loeffler, J.-P., et al. (2008). Dyslipidemia is a protective factor in amyotrophic lateral sclerosis. Neurology 70, 1004-1009.

Fromenty, B., Robin, M.A., Igoudjil, A., Mansouri, A., and Pessayre, D. (2004). The ins and outs of mitochondrial dysfunction in NASH. Diabetes Metab. 30, 121-138.

GERBABA, T.K., GREEN-HARRISON, L., and BURET, A.G. (2018). Modeling Host-Microbiome Interactions in Caenorhabditis elegans. Journal of Nematology 49, 348-356.

Gidalevitz, T., Ben-Zvi, A., Ho, K.H., Brignull, H.R., and Morimoto, R.I. (2006). Progressive disruption of cellular protein folding in models of polyglutamine diseases. Science 311, 1471-1474.

Glatz, J.F.C., and Luiken, J.J.F.P. (2017). From fat to FAT (CD36/SR-B2): Understanding the regulation of cellular fatty acid uptake. Biochimie 136, 21-26. 
Gupta, G., Qin, H., and Song, J. (2012). Intrinsically unstructured domain 3 of hepatitis C Virus NS5A forms a "fuzzy complex" with VAPB-MSP domain which carries ALS-causing mutations. PLoS ONE 7, e39261.

Harding, A., Gonder, U., Robinson, S.J., Crean, S., and Singhrao, S.K. (2017). Exploring the Association between Alzheimer's Disease, Oral Health, Microbial Endocrinology and Nutrition. Front Aging Neurosci 9, 1335.

Hogg, M.C., Halang, L., Woods, I., Coughlan, K.S., and Prehn, J.H.M. (2018). Riluzole does not improve lifespan or motor function in three ALS mouse models. Amyotroph Lateral Scler Frontotemporal Degener 19, 438-445.

Jawaid, A., Khan, R., Polymenidou, M., and Schulz, P.E. (2018). Diseasemodifying effects of metabolic perturbations in ALS/FTLD. Mol Neurodegener 13, 63.

Kim, D.-G., Krenz, A., Toussaint, L.E., Maurer, K.J., Robinson, S.-A., Yan, A., Torres, L., and Bynoe, M.S. (2016). Non-alcoholic fatty liver disease induces signs of Alzheimer's disease (AD) in wild-type mice and accelerates pathological signs of $A D$ in an $A D$ model. J Neuroinflammation 13, 1.

Kira, Y., Nishikawa, M., Ochi, A., Sato, E., and Inoue, M. (2006). L-carnitine suppresses the onset of neuromuscular degeneration and increases the life span of mice with familial amyotrophic lateral sclerosis. Brain Res. 1070, 206-214.

Lawton, K.A., Brown, M.V., Alexander, D., Li, Z., Wulff, J.E., Lawson, R., Jaffa, M., Milburn, M.V., Ryals, J.A., Bowser, R., et al. (2014). Plasma metabolomic biomarker panel to distinguish patients with amyotrophic lateral sclerosis from disease mimics. Amyotroph Lateral Scler Frontotemporal Degener 15, 362-370.

Lawton, K.A., Cudkowicz, M.E., Brown, M.V., Alexander, D., Caffrey, R., Wulff, J.E., Bowser, R., Lawson, R., Jaffa, M., Milburn, M.V., et al. (2012). Biochemical alterations associated with ALS. Amyotroph Lateral Scler 13, 110-118.

Lee, S.-J., Zhang, J., Choi, A.M.K., and Kim, H.P. (2013). Mitochondrial dysfunction induces formation of lipid droplets as a generalized response to stress. Oxid Med Cell Longev 2013, 327167-10.

Lee, S.H., and Yang, E.J. (2018). Relationship between Liver Pathology and Disease Progression in a Murine Model of Amyotrophic Lateral Sclerosis. Neurodegener Dis 18, 200-207.

Li, B., and Dewey, C.N. (2011). RSEM: accurate transcript quantification from RNA-Seq data with or without a reference genome. BMC Bioinformatics 12, 57.

Love, M.I., Huber, W., and Anders, S. (2014). Moderated estimation of fold change and dispersion for RNA-seq data with DESeq2. Genome Biol. 15, 31. 
Lynch, S.V., and Pedersen, O. (2016). The Human Intestinal Microbiome in Health and Disease. N. Engl. J. Med. 375, 2369-2379.

Manzo, E., O'Conner, A.G., Barrows, J.M., Shreiner, D.D., Birchak, G.J., and Zarnescu, D.C. (2018). Medium-Chain Fatty Acids, Beta-Hydroxybutyric Acid and Genetic Modulation of the Carnitine Shuttle Are Protective in a Drosophila Model of ALS Based on TDP-43. Front Mol Neurosci 11, 182.

Matey-Hernandez, M.L., Williams, F.M.K., Potter, T., Valdes, A.M., Spector, T.D., and Menni, C. (2018). Genetic and microbiome influence on lipid metabolism and dyslipidemia. Physiological Genomics 50, 117-126.

Mi, H., Huang, X., Muruganujan, A., Tang, H., Mills, C., Kang, D., and Thomas, P.D. (2017). PANTHER version 11: expanded annotation data from Gene Ontology and Reactome pathways, and data analysis tool enhancements. Nucleic Acids Res. 45, D183-D189.

Miller, R.G., Mitchell, J.D., and Moore, D.H. (2012). Riluzole for amyotrophic lateral sclerosis (ALS)/motor neuron disease (MND). Cochrane Database Syst Rev 3, CD001447.

Morley, J.F., Brignull, H.R., Weyers, J.J., and Morimoto, R.I. (2002). The threshold for polyglutamine-expansion protein aggregation and cellular toxicity is dynamic and influenced by aging in Caenorhabditis elegans. Proceedings of the National Academy of Sciences 99, 10417-10422.

Mulak, A., and Bonaz, B. (2015). Brain-gut-microbiota axis in Parkinson's disease. World J. Gastroenterol. 21, 10609-10620.

Nagase, M., Yamamoto, Y., Miyazaki, Y., and Yoshino, H. (2016). Increased oxidative stress in patients with amyotrophic lateral sclerosis and the effect of edaravone administration. Redox Rep. 21, 104-112.

Nash, L.A., Burns, J.K., Chardon, J.W., Kothary, R., and Parks, R.J. (2016). Spinal Muscular Atrophy: More than a Disease of Motor Neurons? Curr. Mol. Med. 16, 779-792.

Nodera, H., Takamatsu, N., Muguruma, N., Ukimoto, K., Nishio, S., Oda, M., Izumi, Y., and Kaji, R. (2015). Frequent hepatic steatosis in amyotrophic lateral sclerosis: Implication for systemic involvement. Neurology and Clinical Neuroscience 3, 5862.

O'Donnell, M.P., Fox, B.W., Chao, P.-H., Schroeder, F.C., and Sengupta, P. (2020). A neurotransmitter produced by gut bacteria modulates host sensory behaviour. Nature 583, 415-420. 
Pasinelli, P., Belford, M.E., Lennon, N., Bacskai, B.J., Hyman, B.T., Trotti, D., and Brown, R.H. (2004). Amyotrophic lateral sclerosis-associated SOD1 mutant proteins bind and aggregate with Bcl-2 in spinal cord mitochondria. Neuron 43, 19-30.

Prudencio, M., Belzil, V.V., Batra, R., Ross, C.A., Gendron, T.F., Pregent, L.J., Murray, M.E., Overstreet, K.K., Piazza-Johnston, A.E., Desaro, P., et al. (2015). Distinct brain transcriptome profiles in C9orf72-associated and sporadic ALS. Nat. Neurosci. 18, 1175-1182.

Roy Sarkar, S., and Banerjee, S. (2019). Gut microbiota in neurodegenerative disorders. Journal of Neuroimmunology 328, 98-104.

Ruan, L., Zhou, C., Jin, E., Kucharavy, A., Zhang, Y., Wen, Z., Florens, L., and Li, R. (2017). Cytosolic proteostasis through importing of misfolded proteins into mitochondria. Nature 543, 443-446.

Schmeisser, K., and Parker, J.A. (2018). Nicotinamide-N-methyltransferase controls behavior, neurodegeneration and lifespan by regulating neuronal autophagy. PLoS Genet. 14, e1007561.

Schulenburg, H., and Félix, M.-A. (2017). The Natural Biotic Environment of Caenorhabditis elegans. Genetics 206, 55-86.

Shamim, A., Mahmood, T., Ahsan, F., Kumar, A., and Bagga, P. (2018). Lipids: An insight into the neurodegenerative disorders. Clinical Nutrition Experimental 20, 119.

Shapira, M. (2017). Host-microbiota interactions in Caenorhabditis elegans and their significance. Current Opinion in Microbiology 38, 142-147.

Sherwin, E., Dinan, T.G., and Cryan, J.F. (2018). Recent developments in understanding the role of the gut microbiota in brain health and disease. Ann. N. Y. Acad. Sci. 1420, 5-25.

Stiernagle, T. (2006). Maintenance of C. elegans. WormBook 1-11.

Takahashi, F., Takei, K., Tsuda, K., and Palumbo, J. (2017). Post-hoc analysis of MCl186-17, the extension study to MCl186-16, the confirmatory double-blind, parallel-group, placebo-controlled study of edaravone in amyotrophic lateral sclerosis. Amyotroph Lateral Scler Frontotemporal Degener 18, 32-39.

Therrien, M., and Parker, J.A. (2014). Worming forward: amyotrophic lateral sclerosis toxicity mechanisms and genetic interactions in Caenorhabditis elegans. Front Genet 5, 85. 
The Gene Ontology Consortium (2019). The Gene Ontology Resource: 20 years and still GOing strong. Nucleic Acids Res. 47, D330-D338.

Urrutia, A., García-Angulo, V.A., Fuentes, A., Caneo, M., Legüe, M., Urquiza, S., Delgado, S.E., Ugalde, J., Burdisso, P., and Calixto, A. (2020). Bacterially produced metabolites protect $\mathrm{C}$. elegans neurons from degeneration. PLoS Biol. 18, e3000638.

Vaccaro, A., Tauffenberger, A., Aggad, D., Rouleau, G., Drapeau, P., and Parker, J.A. (2012). Mutant TDP-43 and FUS cause age-dependent paralysis and neurodegeneration in C. elegans. PLoS ONE 7, e31321.

Vande Velde, C., Miller, T.M., Cashman, N.R., and Cleveland, D.W. (2008). Selective association of misfolded ALS-linked mutant SOD1 with the cytoplasmic face of mitochondria. Proc. Natl. Acad. Sci. U.S.a. 105, 4022-4027.

Vercruysse, P., Vieau, D., Blum, D., Petersén, Å., and Dupuis, L. (2018). Hypothalamic Alterations in Neurodegenerative Diseases and Their Relation to Abnormal Energy Metabolism. Front Mol Neurosci 11, 2.

Vérièpe, J., Fossouo, L., and Parker, J.A. (2015). Neurodegeneration in C. elegans models of ALS requires TIR-1/Sarm1 immune pathway activation in neurons. Nat Commun 6, 7319.

Vishwanath, V.A. (2016). Fatty Acid Beta-Oxidation Disorders: A Brief Review. Ann Neurosci 23, 51-55.

Vogt, N.M., Kerby, R.L., Dill-McFarland, K.A., Harding, S.J., Merluzzi, A.P., Johnson, S.C., Carlsson, C.M., Asthana, S., Zetterberg, H., Blennow, K., et al. (2017). Gut microbiome alterations in Alzheimer's disease. Sci Rep 7, 595.

Watson, E., MacNeil, L.T., Arda, H.E., Zhu, L.J., and Walhout, A.J.M. (2013). Integration of metabolic and gene regulatory networks modulates the $C$. elegans dietary response. Cell 153, 253-266.

Watson, E., MacNeil, L.T., Ritter, A.D., Yilmaz, L.S., Rosebrock, A.P., Caudy, A.A., and Walhout, A.J.M. (2014). Interspecies Systems Biology Uncovers Metabolites Affecting C. elegans Gene Expression and Life History Traits. Cell 156, 759-770.

Watts, J.L., and Ristow, M. (2017). Lipid and Carbohydrate Metabolism in Caenorhabditis elegans. Genetics 207, 413-446.

Wong, M.W., Braidy, N., Poljak, A., Pickford, R., Thambisetty, M., and Sachdev, P.S. (2017). Dysregulation of lipids in Alzheimer's disease and their role as potential biomarkers. Alzheimers Dement 13, 810-827. 
Wu, S., Yi, J., Zhang, Y.-G., Zhou, J., and Sun, J. (2015). Leaky intestine and impaired microbiome in an amyotrophic lateral sclerosis mouse model. Physiol Rep 3, e12356-e12356.

Zhang, Y.-G., Wu, S., Yi, J., Xia, Y., Jin, D., Zhou, J., and Sun, J. (2017). Target Intestinal Microbiota to Alleviate Disease Progression in Amyotrophic Lateral Sclerosis. Clin Ther 39, 322-336.

Zolkipli, Z., Sherlock, M., Biggar, W.D., Taylor, G., Hutchison, J.S., Peliowski, A., Alman, B.A., Ling, S.C., and Tein, I. (2012). Abnormal fatty acid metabolism in spinal muscular atrophy may predispose to perioperative risks. Eur. J. Paediatr. Neurol. 16, 549-553. 
Table 1 : List of probiotics from Lallemand Health Solutions Individual bacterial strains

Bifidobacterium animalis subsp. lactis B94

Bifidobacterium breve HA-129

Bacillus subtilis R0179

Lacticaseibacillus plantarum R1012

Lacticaseibacillus plantarum HA-119

Lacticaseibacillus casei L26

Lacticaseibacillus paracasei HA-196

Lacticaseibacillus helveticus R0052

Lacticaseibacillus rhamnosus R0011

Lacticaseibacillus rhamnosus R0343

Lacticaseibacillus rhamnosus HA-114

Lacticaseibacillus rhamnosus $\mathrm{HA}-111$

Pediococcus acidilactici R1001

\section{Blends}

L. plantarum HA-119 and B. animalis subsp. lactis B94 (1:1)

L. rhamnosus HA-114 and $B$. animalis subsp. lactis B94 (1:1)

L. rhamnosus HA-114 and L. plantarum HA-119 (1:1) 
A

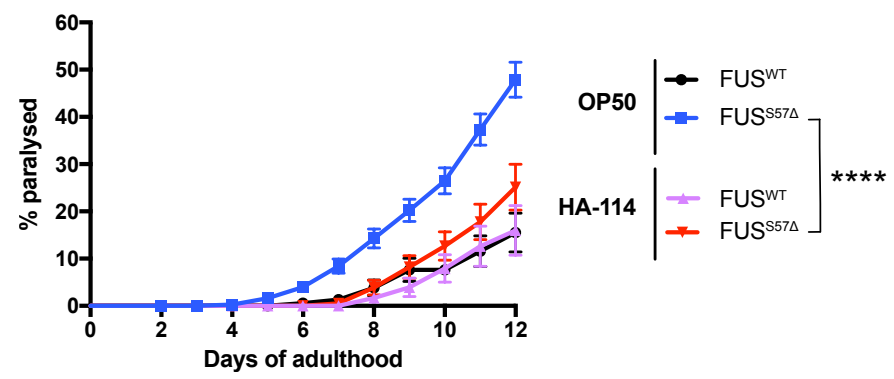

C

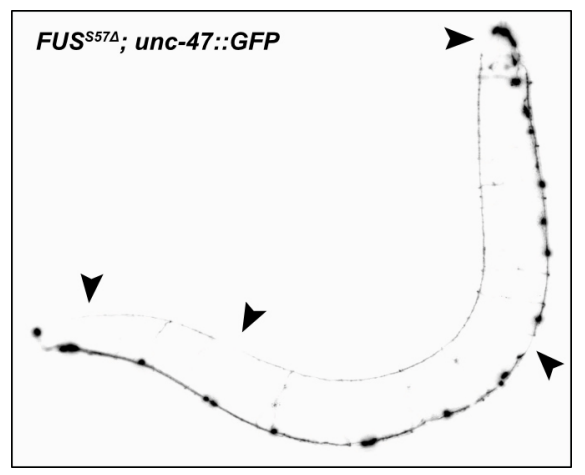

F

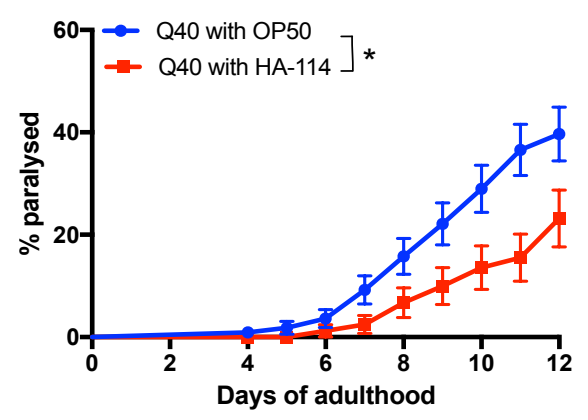

D

G
B
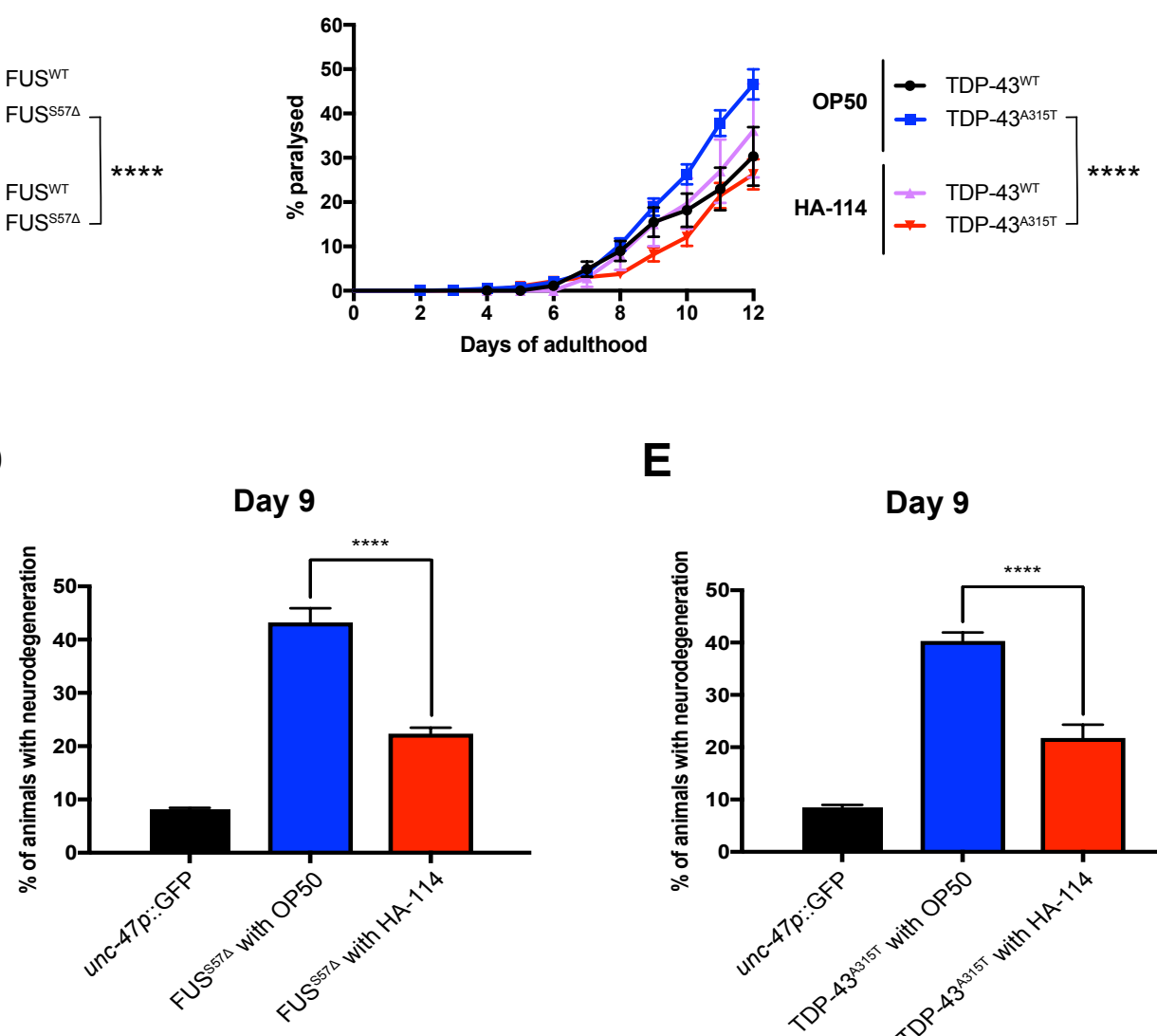

E
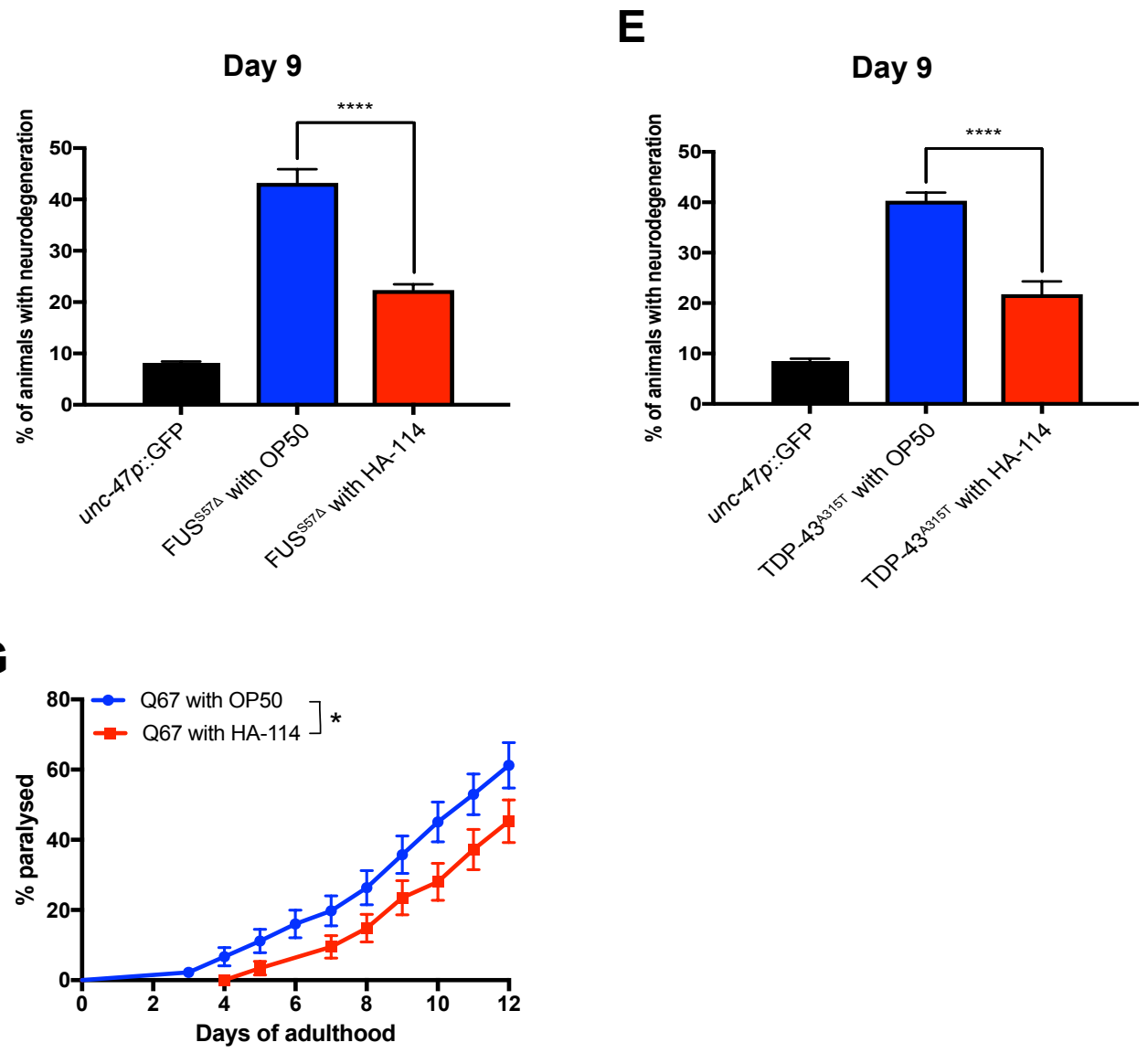
Figure 1. Lacticaseibacillus rhamnosus HA-114 rescues motor defects and neurodegeneration. Transgenics were monitored from the adult stage, scored daily for paralysis and fed with control OP50 or HA-114. (A) Mutant FUS worms fed with HA-114 showed less paralysis compared to transgenics expressing mutant FUS fed with OP50. (B) Transgenics expressing mutant TDP-43 fed with probiotics showed a lower rate of progressive paralysis than transgenics expressing mutant TDP-43 fed with OP50. (C) Image of a whole FUS ${ }^{\triangle S 57}$ worm expressing GFP in the GABAergic motor neurons. mFUS transgenics display gaps along neuronal processes (arrows). (D) Mutant FUS worms fed with HA-114 have a similar rate of neurodegeneration compared to transgenic GFP controls at day 9. (E) Mutant TDP-43 transgenics fed with probiotics had a lower rate of neurodegeneration at day 9 compared to mutant TDP-43 transgenics fed with OP50. (F) HA-114 rescued aged-dependent paralysis phenotype in transgenics expressing Q40 and in Q67 (G) animals. *: $p<0.05,{ }^{* * * *}$ : $<<0.0001$. 
A
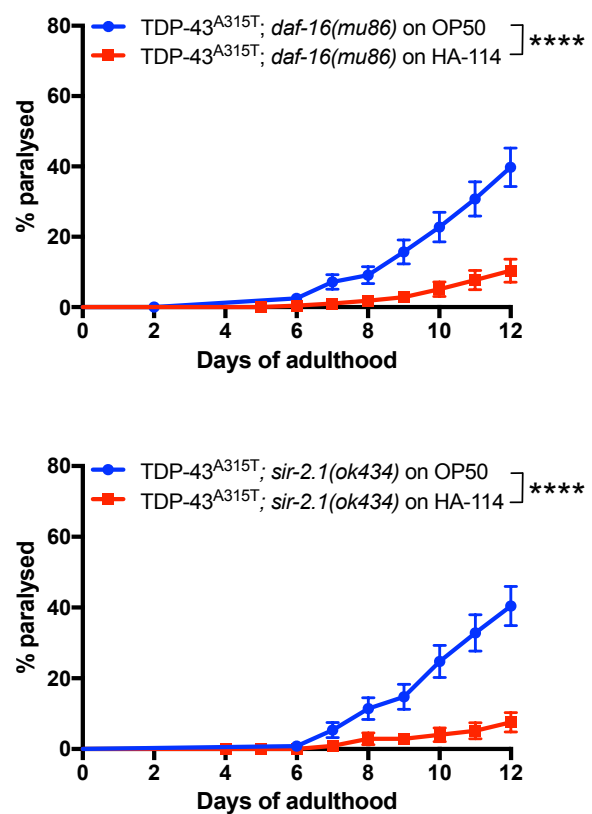

C
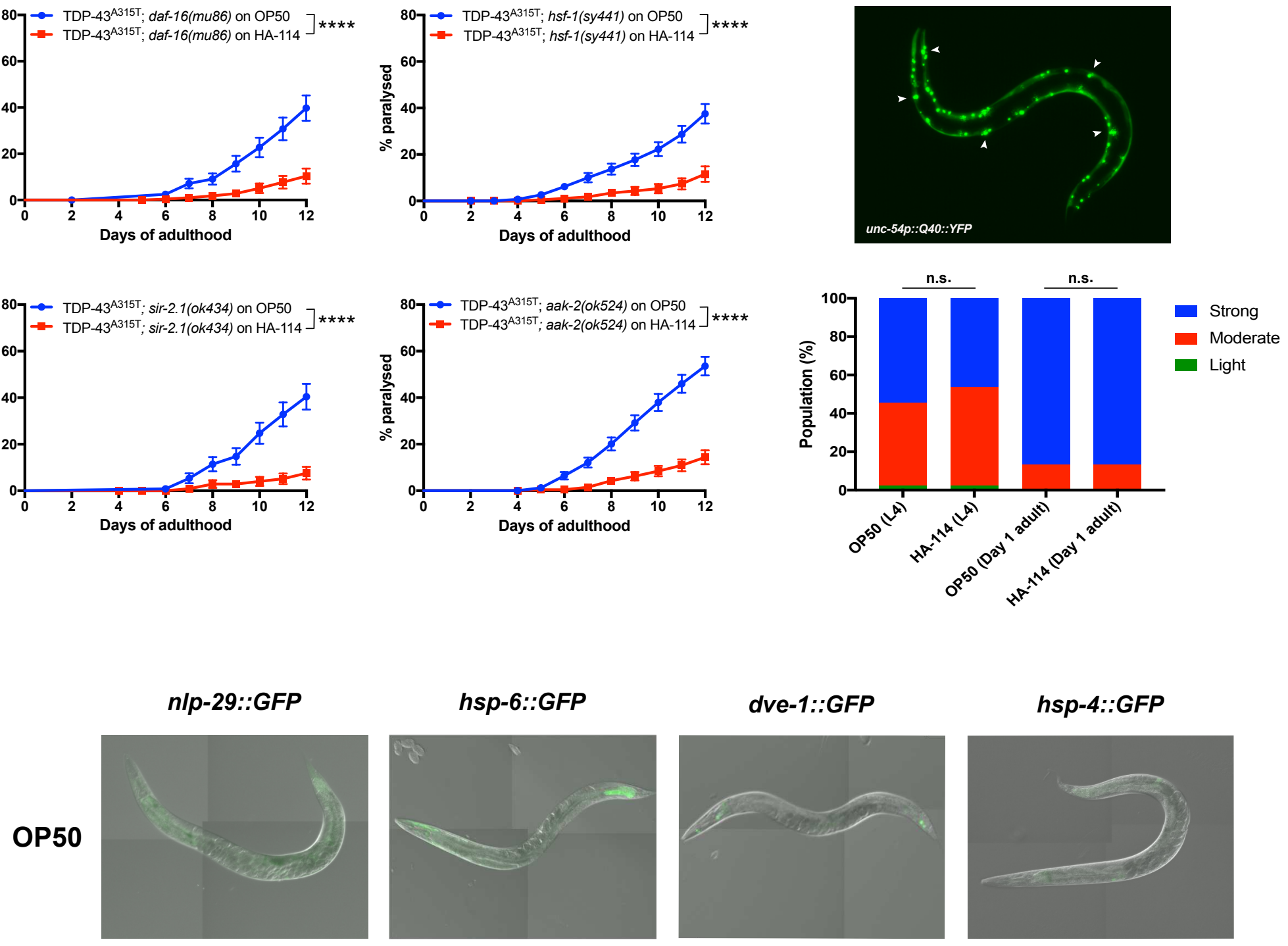

B

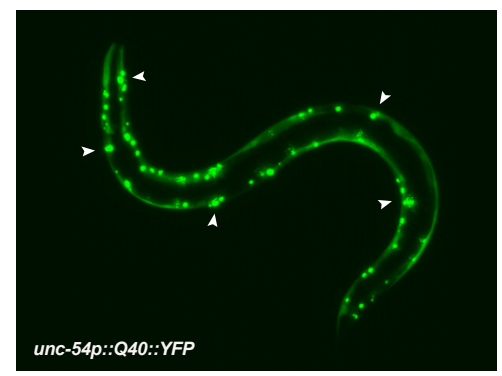

$c-54 p:: Q 40:: Y F P$

dve-1::GFP

hsp-4::GFP
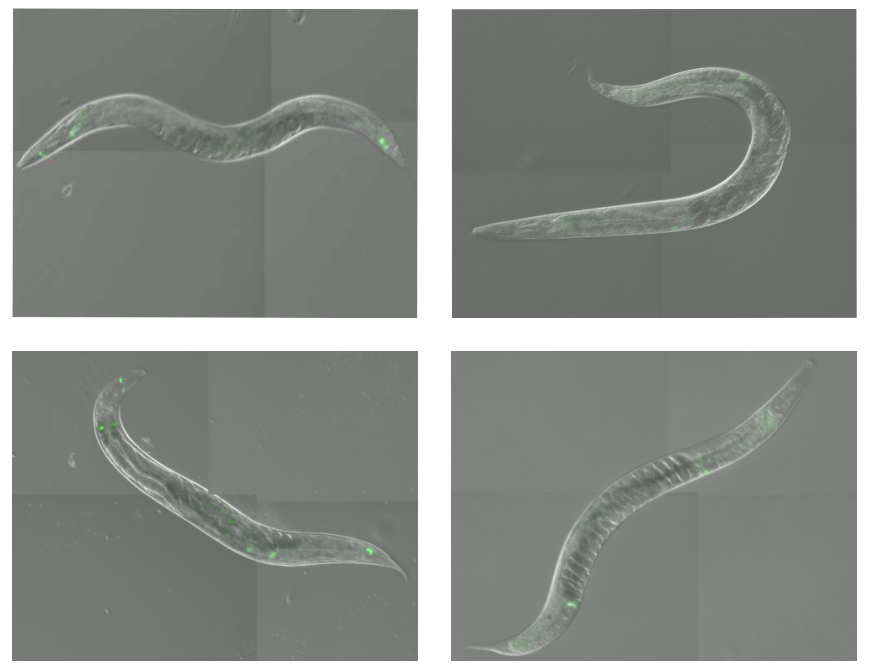

HA-114

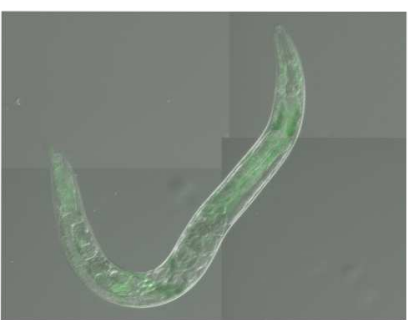

hsp-6::GFP
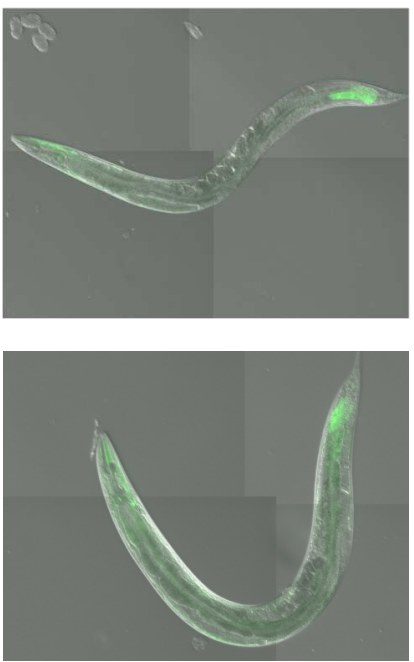
Figure 2. HA-114 does not require classic stress and metabolic pathways in C. elegans for neuroprotection. Neuroprotection provided by L. rhamnosus HA114 was unaffected by (A) daf-16(mu86) deletion, nor by hsf-1(sy441) point mutation. Both sir-2.1 and aak-2 genes are not required for neuroprotection granted by HA-114 probiotics. (B) Aggregation was not altered by HA-114 in a polyQ model at L4 or at day 1 of adulthood. (C) HA-114 did not affect GFP expression of key genes of innate immune response (n/p-29), UPR ${ }^{\text {mito }}$ response (hsp-6 and $d v e-1)$ or UPR ${ }^{\mathrm{ER}}(h s p-4)$. 
A
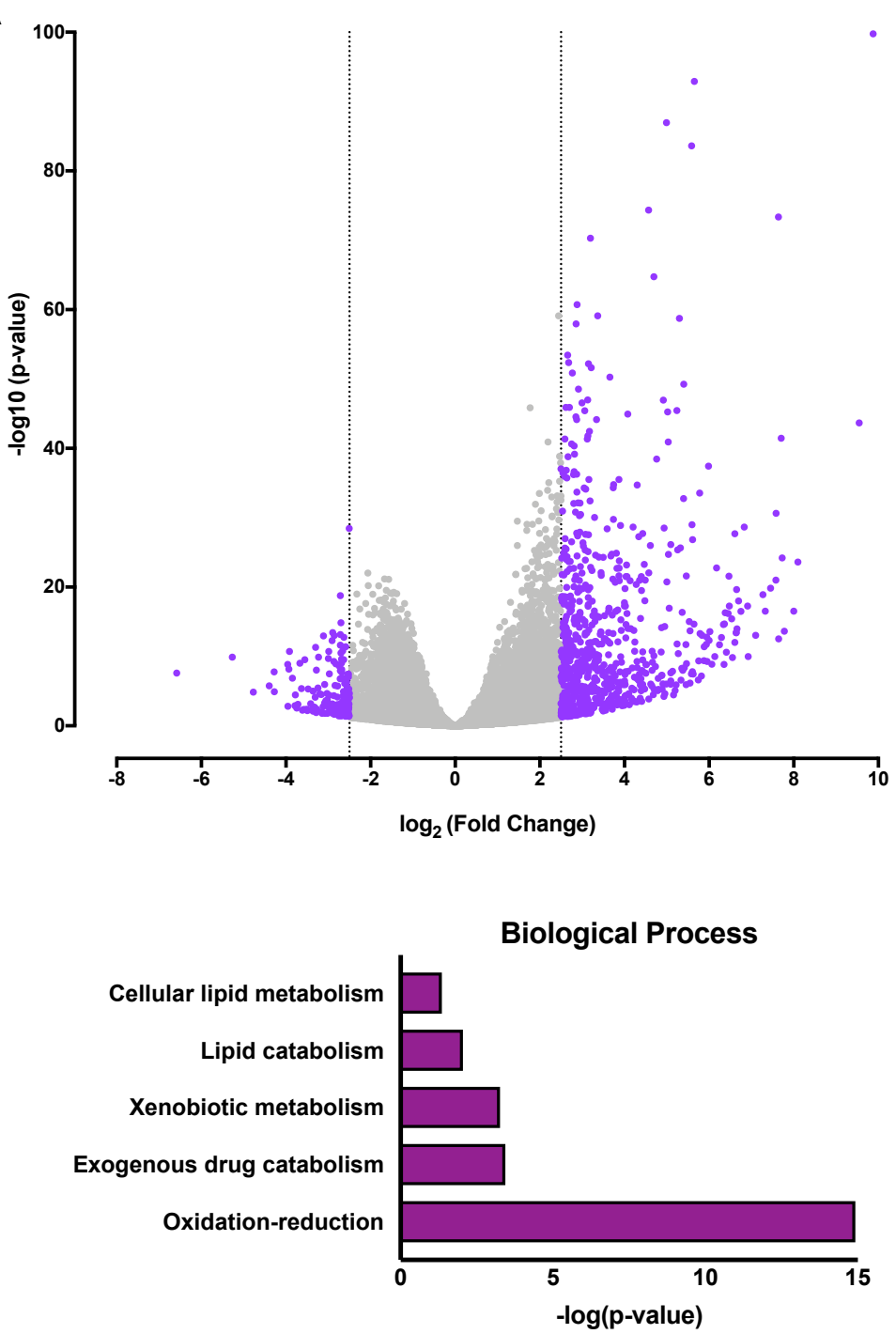

B
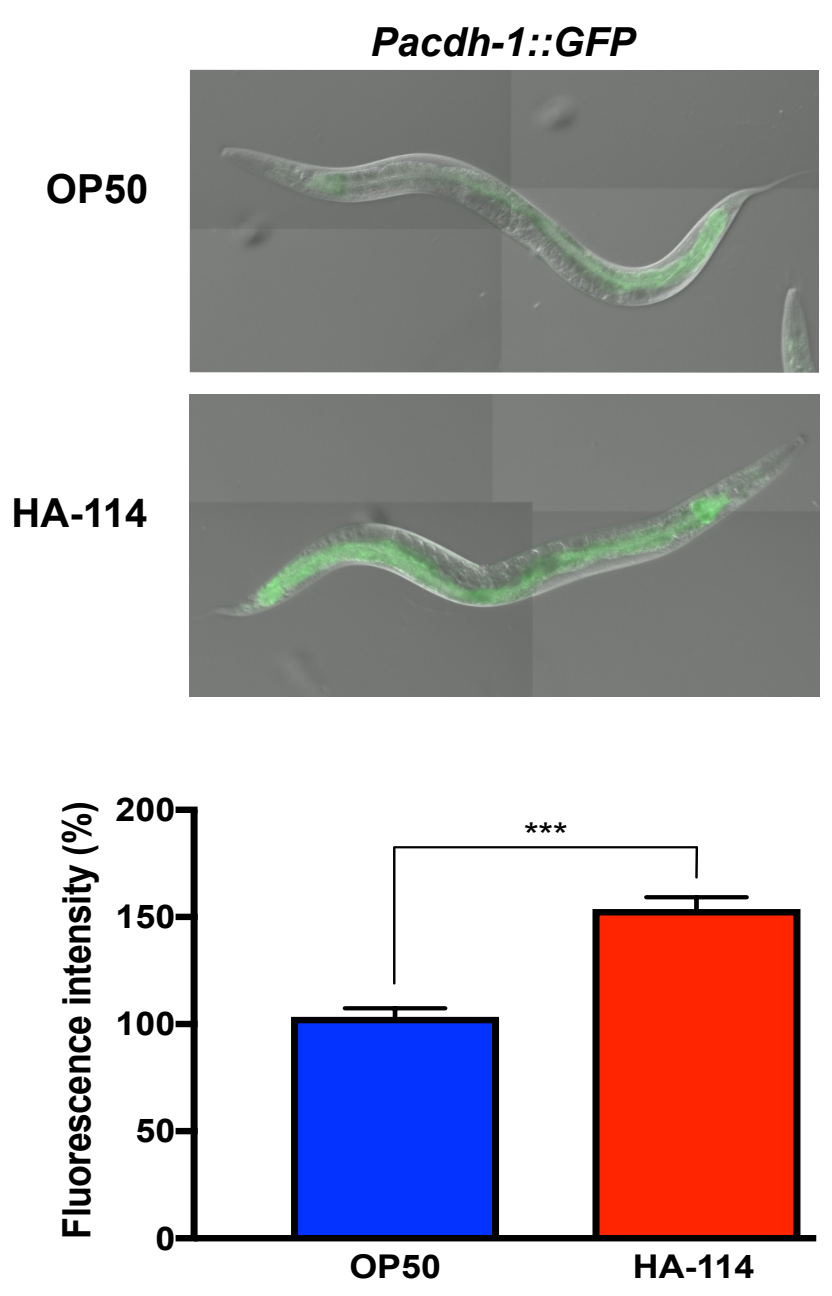

C

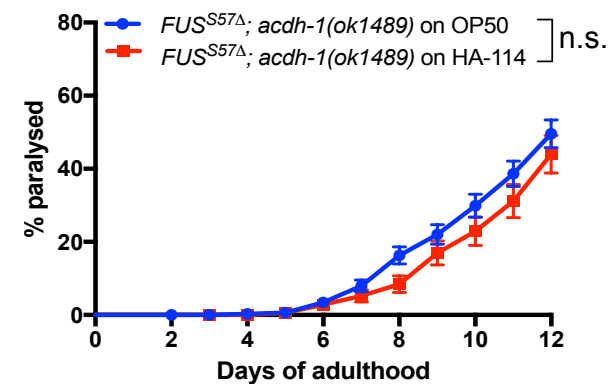

D

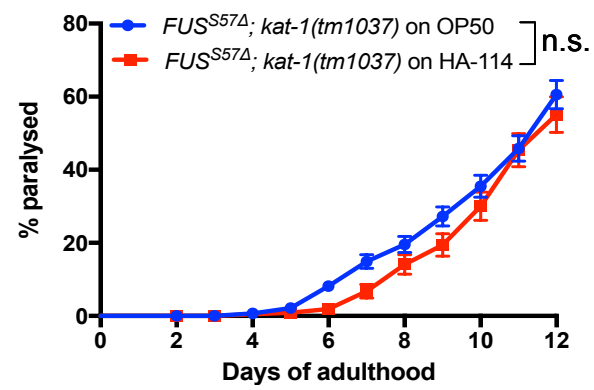

E

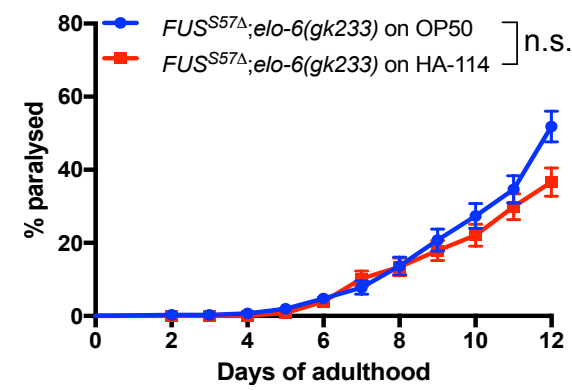


Figure 3. acdh-1, kat-1 and elo-6 are essential for neuroprotection provided by HA-114. (A) Volcano plot of RNA-Seq data of N2 worms fed with Lacticaseibacillus rhamnosus HA-114 and compared to worms fed with OP50. The data for all genes were plotted as $\log 2$ fold change versus -log10 of the adjusted p-value. Gene ontology (GO) term analysis of the differentially overexpressed genes induced by HA-114 treatment. The GO term analysis was performed with PANTHER 11 by using $C$. elegans genes as background. Only the biological process terms with an enrichment of $P$-value $<0.01$ are shown in this figure. (B) $L$. rhamnosus HA-114 significantly increase GFP expression in pacdh-1::GFP worms when compared to the same worms fed with OP50. Deletion of acdh-1 (C), kat-1 (D) and elo-6 (E) altered the beneficial effect of HA-114. ${ }^{* * *}$ : $p<0.001$. 
A

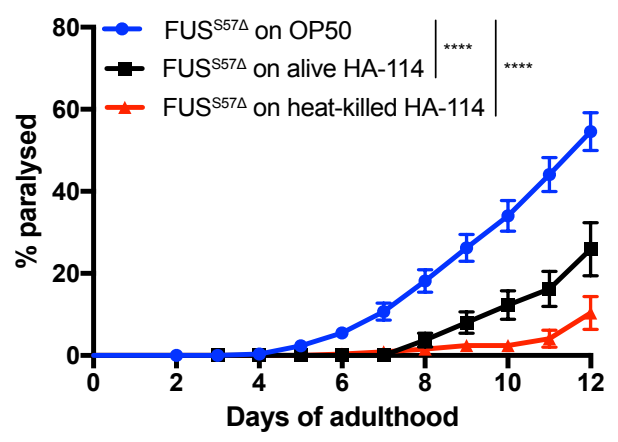

D

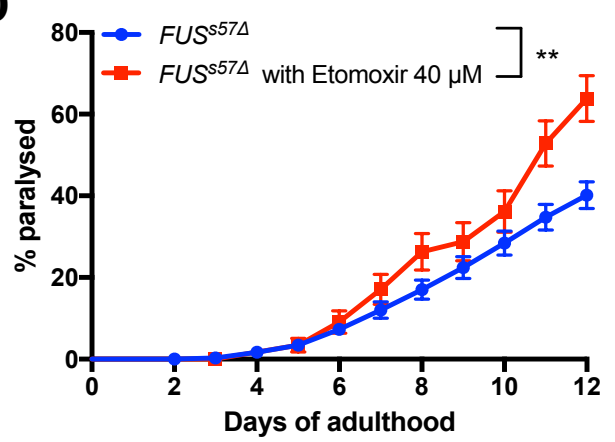

B

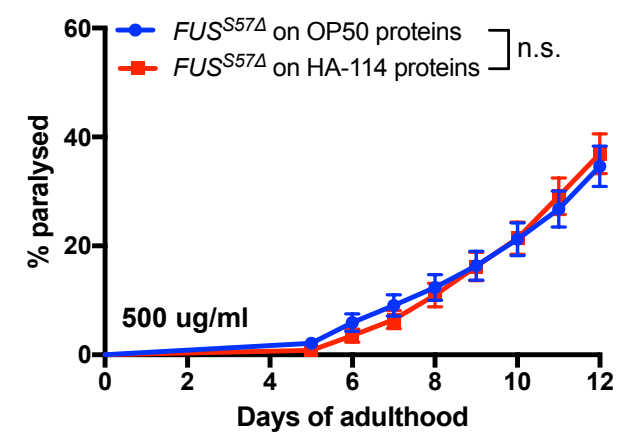

C

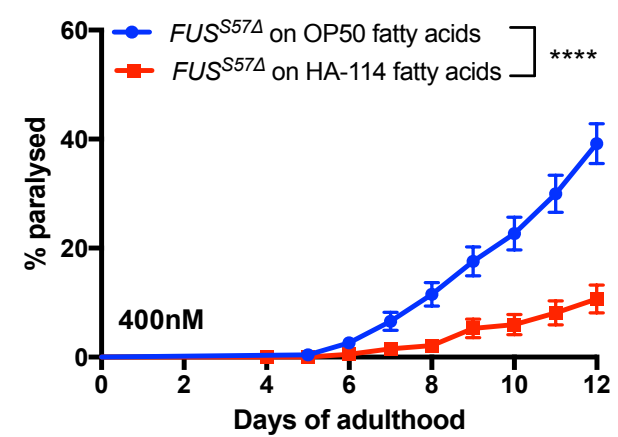

E

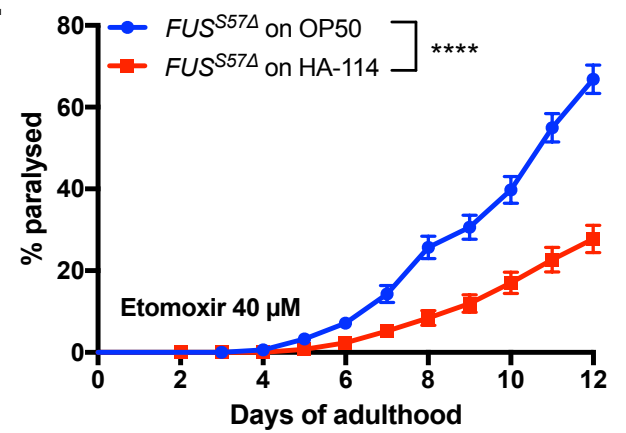

F

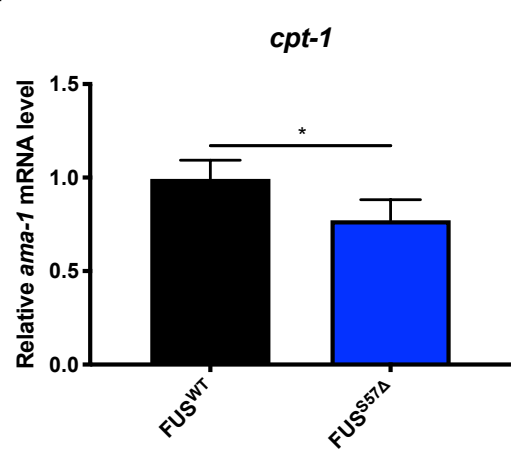

G

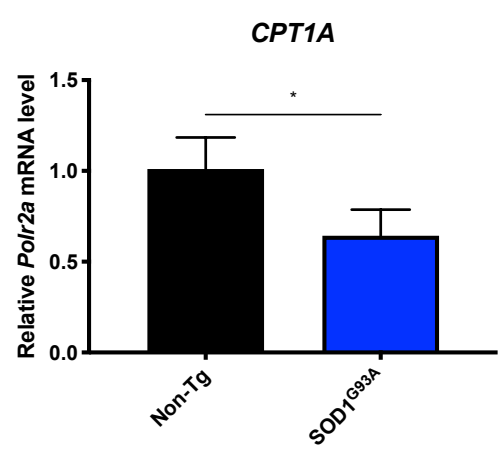

H

CHKB_CPT1B

CPT1C Dataset c9ALS.Ce Healthy.Ce 官 sALS.Ce
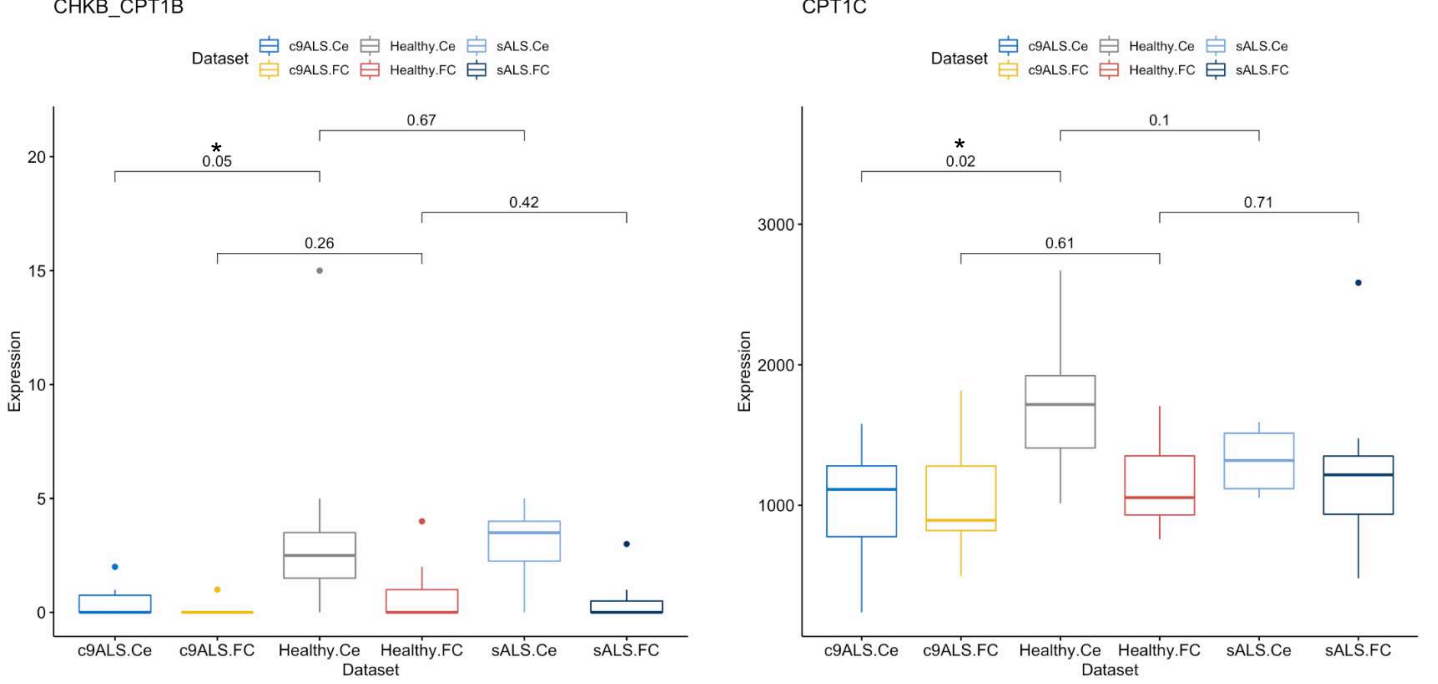

\section{Labarre et al. Figure 4}


Figure 4. HA-114 fatty acids extracts, but not proteins, are sufficient to rescue paralysis. Transgenics were monitored from the adult stage, scored daily for paralysis and fed with control OP50 or HA-114's individual components. (A) Mutant FUS worms fed with heat-killed L. rhamnosus HA-114 did not show paralysis phenotypes when compared to worms fed with OP50. (B) Protein extract from $L$. rhamnosus HA-114 failed to rescue age-dependent paralysis in ALS worm models. (C) When compared to fatty acid extract from OP50, fatty acids extract from HA-114 suppressed paralysis phenotypes in FUS animals. (D) $40 \mu \mathrm{M}$ of Etomoxir, a cpt-1/CPT1 inhibitor, increased paralysis in FUS transgenics. (E) The same concentration was not enough to block neuroprotective effects of $L$. rhamnosus HA-114, when compared to worms fed with OP50. (F) mRNA expression of $c p t-1$ was significantly decreased in $\mathrm{FUS}^{\Delta S 57}$ worms when compared to FUS ${ }^{W T}$ animals. (G) mRNA expression of CPT1A was significantly decreased in livers of SOD $1^{\text {G93A }}$ mice when compared to livers of Non-Tg animals. (H) Decrease expression of two transcripts related to carnitine palmitoyltransferase (CHKB_CPT1B and CPT1C) is observed in C9ORF72 patients when compared to controls. No significant change is observed in sporadic ALS. Data: GEO accession: GSM1642314; SRA study: SRP056477. (Prudencio et al., 2015). *: $p<0.05,{ }^{* *}: p<$ $0.01,{ }^{* * *}: p<0.0001$. 
A

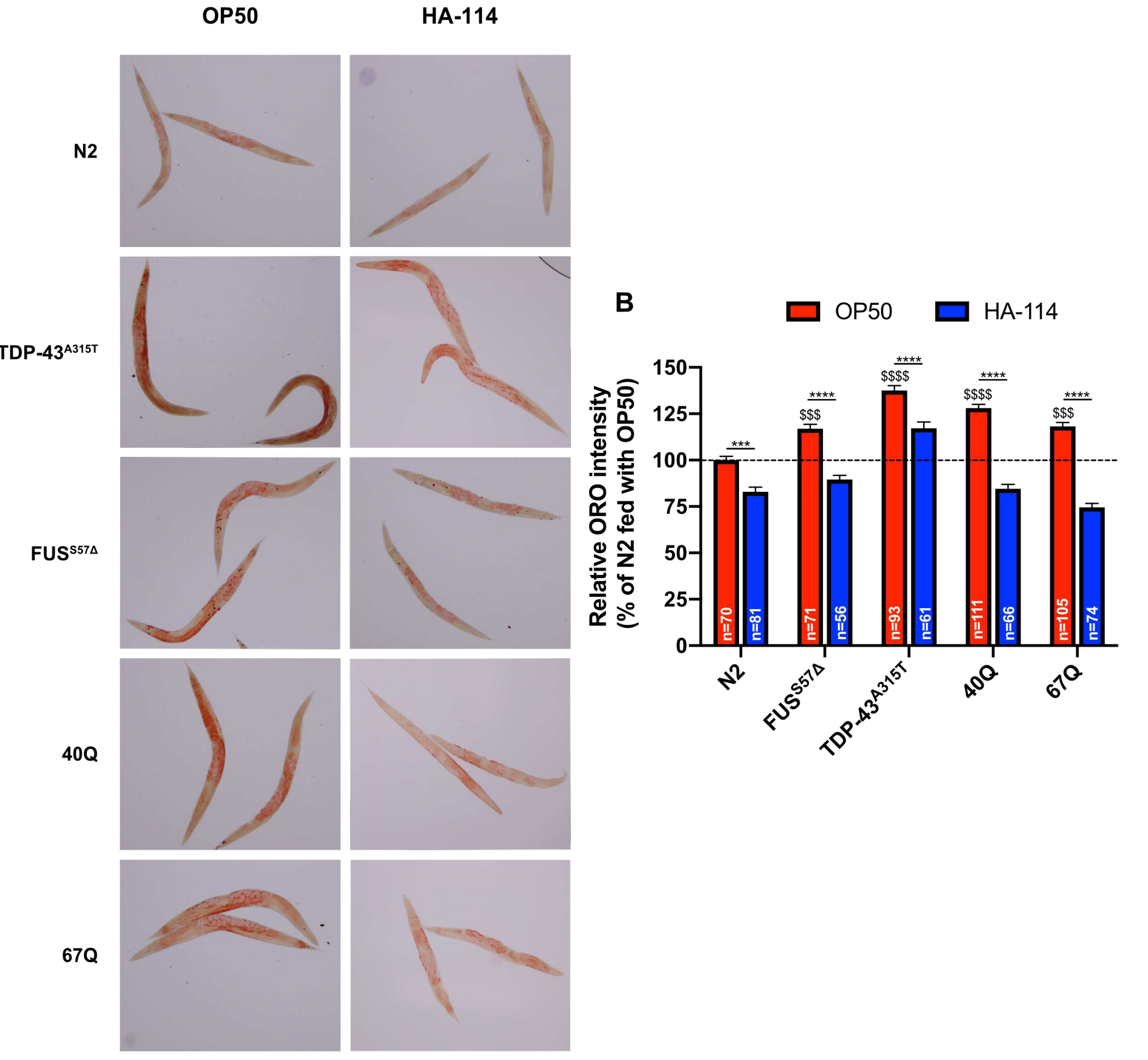


Figure 5. Lacticaseibacillus rhamnosus HA-114 modulate lipid accumulation in worm age-dependent neurodegeneration models. (A) Representative images of worms fed with OP50 and HA-114 and stained with Oil Red O. N2 worms show a basal level of lipid accumulation. (B) Quantification of Oil Red O show increased fat accumulation in worms expressing FUS ${ }^{\Delta S 57}$, TDP-43 ${ }^{A 315 T}, A \beta_{1-42}$ and polyQ expansion (40Q and 67Q) when compared to N2. Worms fed with HA-114 showed a significant decrease of lipid accumulation. \$: statistical difference when compared to $\mathrm{N} 2,{ }^{*}$ : statistical difference within group. ${ }^{* * *}$ or $\$ \$ \$: p<0.001,{ }^{* * * *}$ or $\$ \$ \$: p<0.0001$. 
A

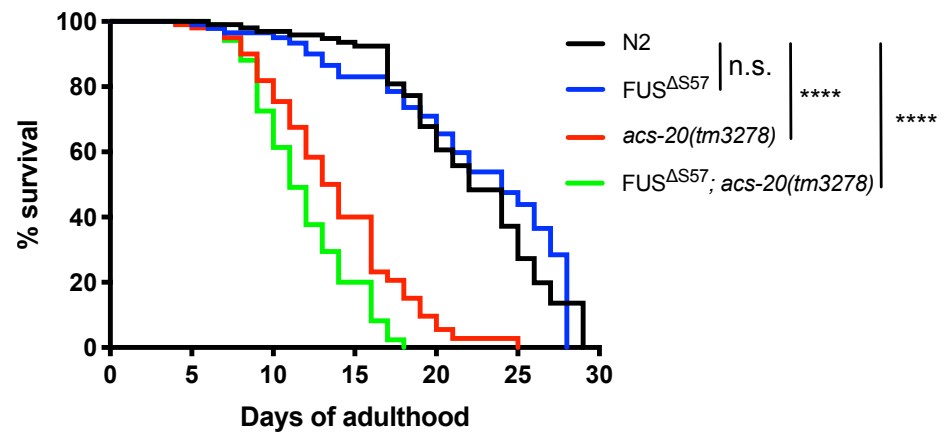

C

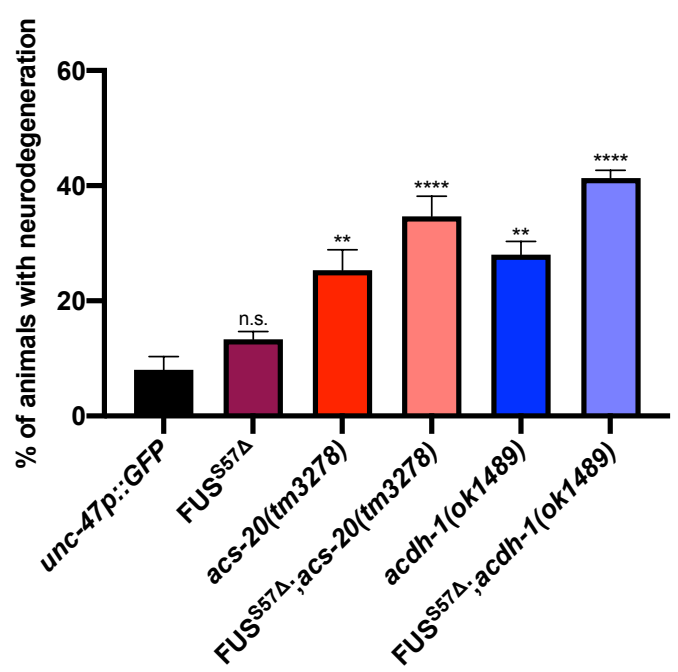

B

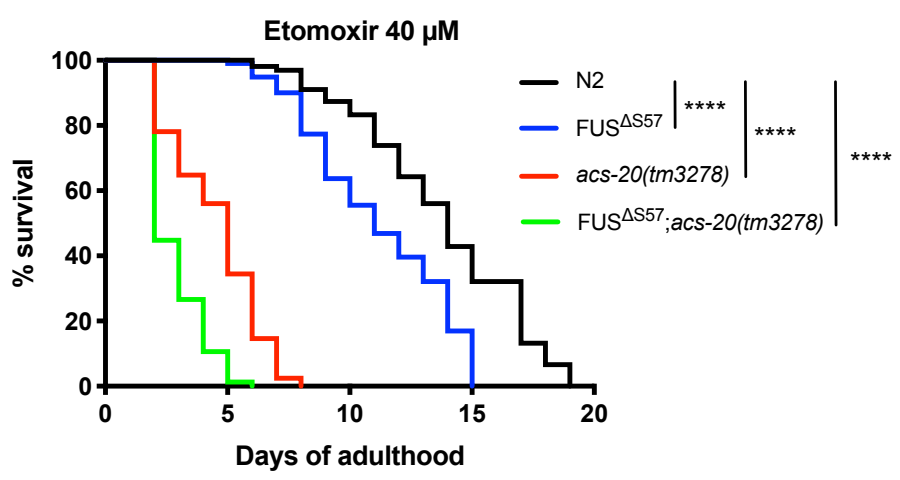


Figure 6. Impaired $\beta$-oxidation contributes to neurodegeneration and shortens lifespan. Worms were monitored from the adult stage until death. (A) acs-20(tm3278) and FUS ${ }^{\Delta S 57}$;acs-20(tm3278) worms have shorter lifespan than $\mathrm{N} 2$ and FUS ${ }^{\triangle S 57}$ animals. (B) Etomoxir $(40 \mu \mathrm{M})$, a cpt-1/CPT1 inhibitor, shorten lifespan of FUS ${ }^{\Delta S 57}$, acs-20(tm3278) and $\mathrm{FUS}^{\Delta S 57}$;acs-20(tm3278) worms when compared to N2. (C) acs-20(tm3278), FUS ${ }^{\Delta S 57}$;acs-20(tm3278), acdh-1(ok1489)

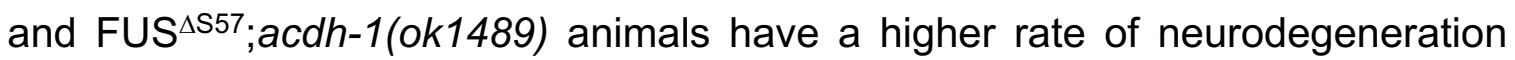
compared to transgenic GFP controls at day $1 .{ }^{* *}: p<0.01,{ }^{* * * *}: p<0.0001$. 
A

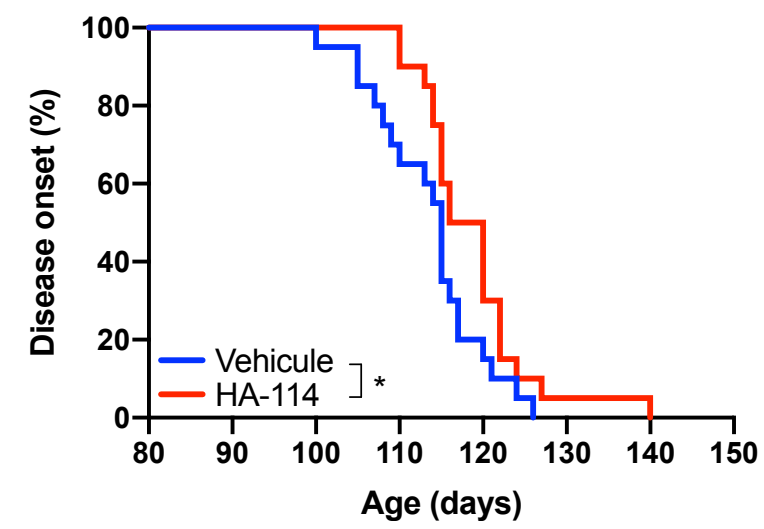

B

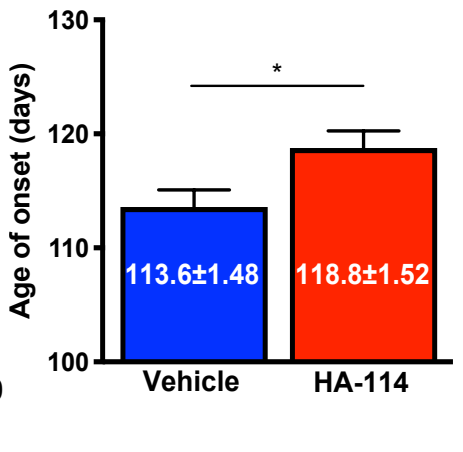

Disease duration

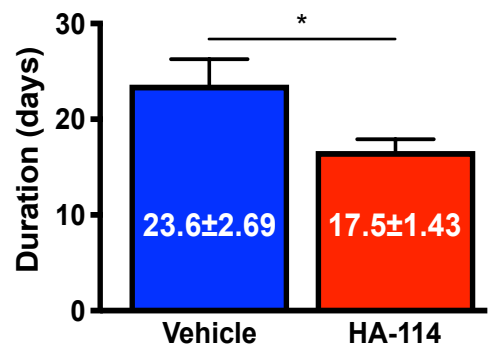

C

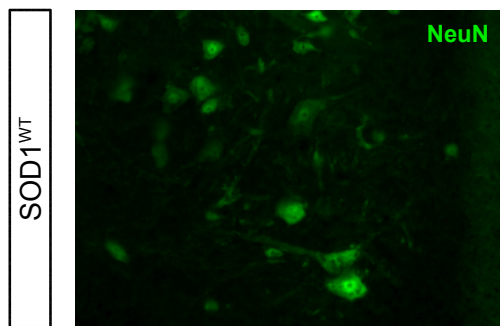

or

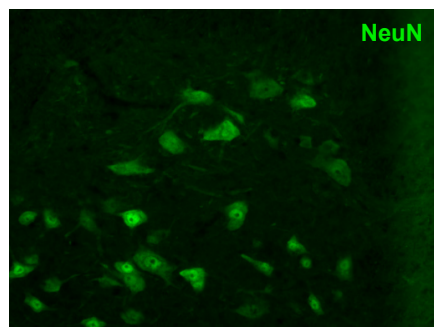

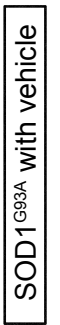

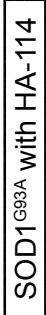
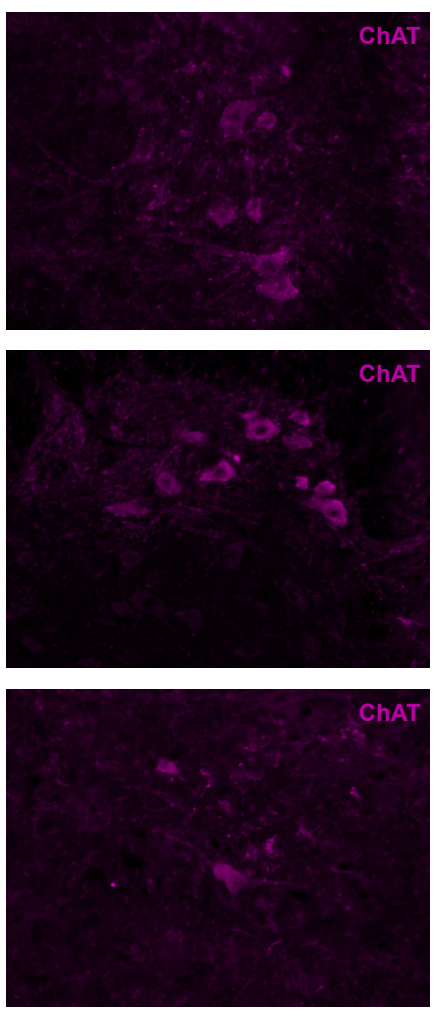

NeuN
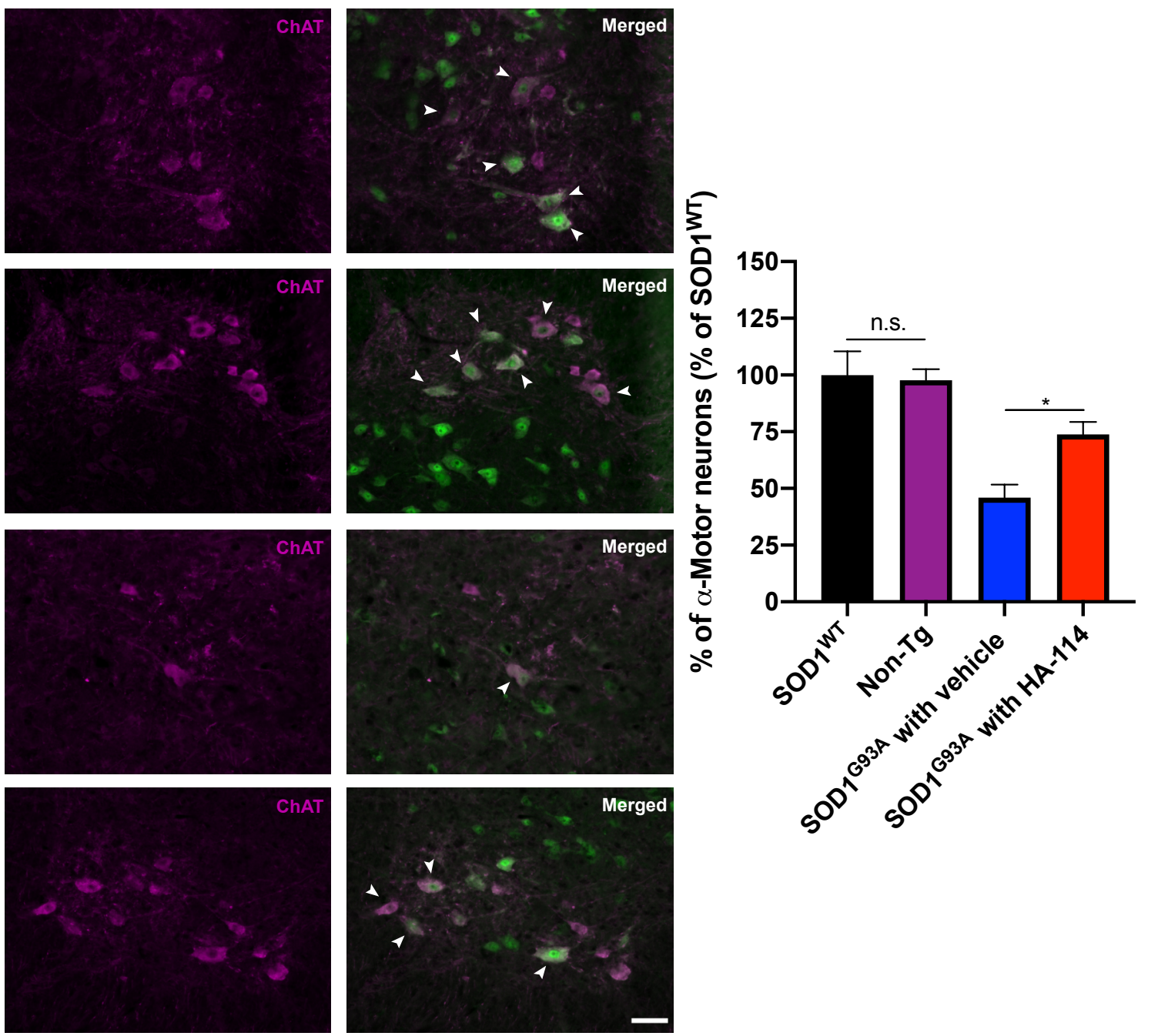

Labarre et al. Figure 7

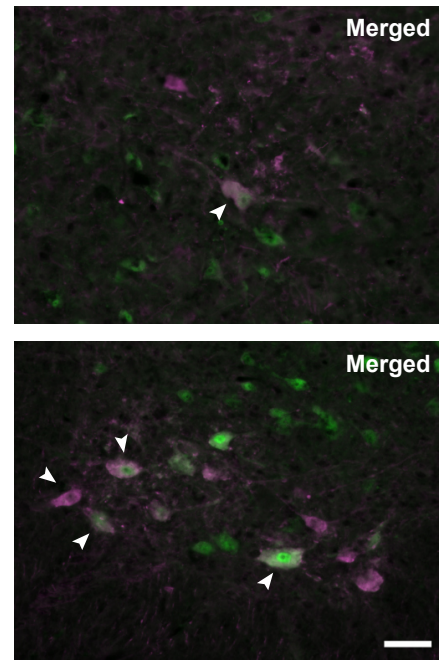


Figure 7. HA-114 delays disease onset in a SOD1 ${ }^{\text {G93A }}$ mouse model. KaplanMeier plot and graph bar showing onset of the disease (A). Onset is delayed in mice treated with $L$. rhamnosus HA-114 (vehicle : $113.6 \pm 1.48$ days, HA-114: 118.8 \pm 1.52 days). (B) Disease duration is also decreased in the group treated with HA-114 (vehicle: $23.6 \pm 2.69$ days, HA-114: 17.5 \pm 1.43 days). 20 mice per group were used. (C) Images of immunofluorescence labeling of motor neurons in the ventral horn of lumbar spinal cord sections; neuronal-specific nuclear protein (NeuN: green) and choline acetyltransferase (ChAT; magenta) were labelled for four different experimental groups at P120 (SOD1 ${ }^{\mathrm{WT}}$, Non-Tg, SOD1 ${ }^{\mathrm{G} 93 \mathrm{~A}}$ with vehicle and SOD1 ${ }^{\text {G93A }}$ with HA-114). Graph bar shows the percentage of double-positive cells $(\mathrm{NeuN}+/ \mathrm{ChAT}+)$, normalized to SOD1 ${ }^{\mathrm{WT}}$ values. HA-114 treatment significantly improved motor neurons loss in SOD $1^{\mathrm{G} 93 \mathrm{~A}}$ mice. Scale bar: $50 \mu \mathrm{m} .{ }^{*}: p<0.05$. 


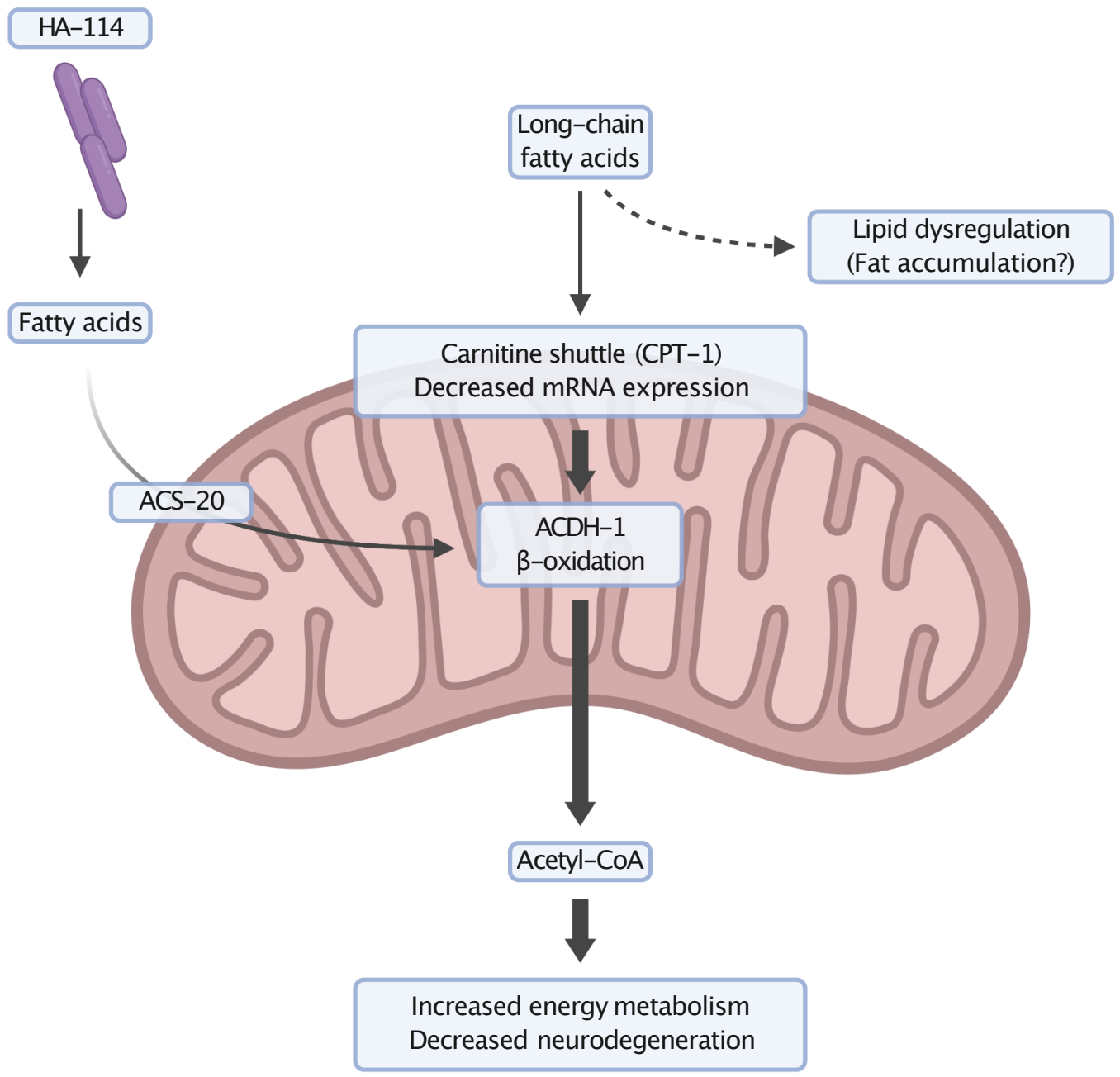


Figure 8. Neuroprotection mechanism of Lacticaseibacillus rhamnosus HA114. ALS models have impaired carnitine shuttle, a mechanism to transport long chain fatty acids across the mitochondrial membrane for energy production via $\beta$ oxidation. Fatty acids, supplied by the probiotic bacteria, is believe to enter the mitochondria independently of the carnitine shuttle to participate in a few rounds of $\beta$-oxidation helping to stabilize energy metabolism, resulting in decreased neurodegeneration and improved lipid homeostasis. 
A

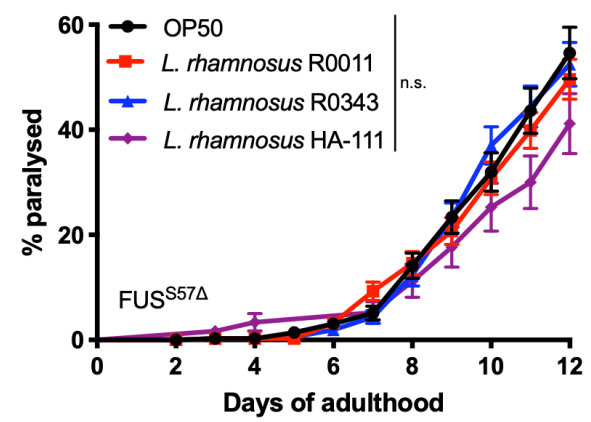

C

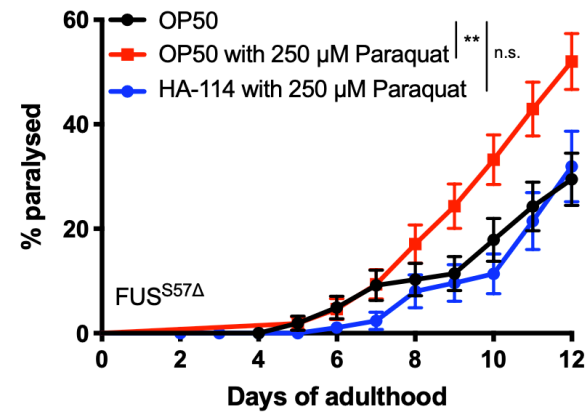

B

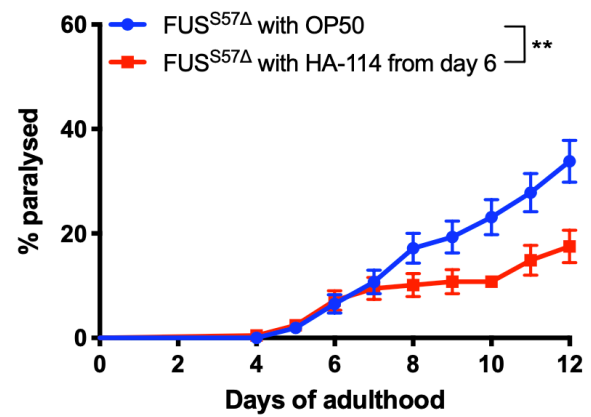

D

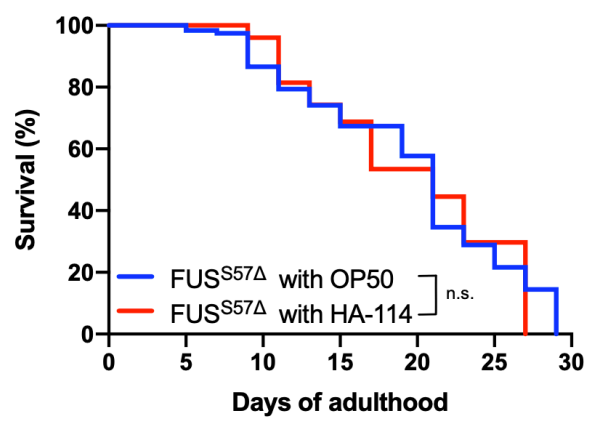

Figure S1. Lacticaseibacillus rhamnosus HA-114 prevents damage from chronic oxidative stress but does not extend lifespan. Transgenics were monitored from the adult stage, scored daily for paralysis. (A) FUS ${ }^{\Delta S 57}$ worms fed with L. rhamnosus strains R0011, R0343 and HA-111 have the same paralysis rate as the worms fed with OP50. (B) Worms fed with HA-114 from day 6 (onset of paralysis) showed less paralysis compared to transgenics expressing mutant FUS fed with OP50. (C) Chronic treatment of $250 \mu \mathrm{M}$ paraquat increased paralysis in FUS ${ }^{\triangle S 57}$ animals. HA-114 supplementation prevents increase of paralysis from paraquat treatment. (D) Transgenics were monitored from the adult stage, scored every two days for mortality and fed with control OP50 or HA-114. Mutant FUS worms fed with HA-114 do not show any lifespan extension when compared to transgenics expressing mutant FUS fed with OP50. ${ }^{* *}: p<0.01$. 

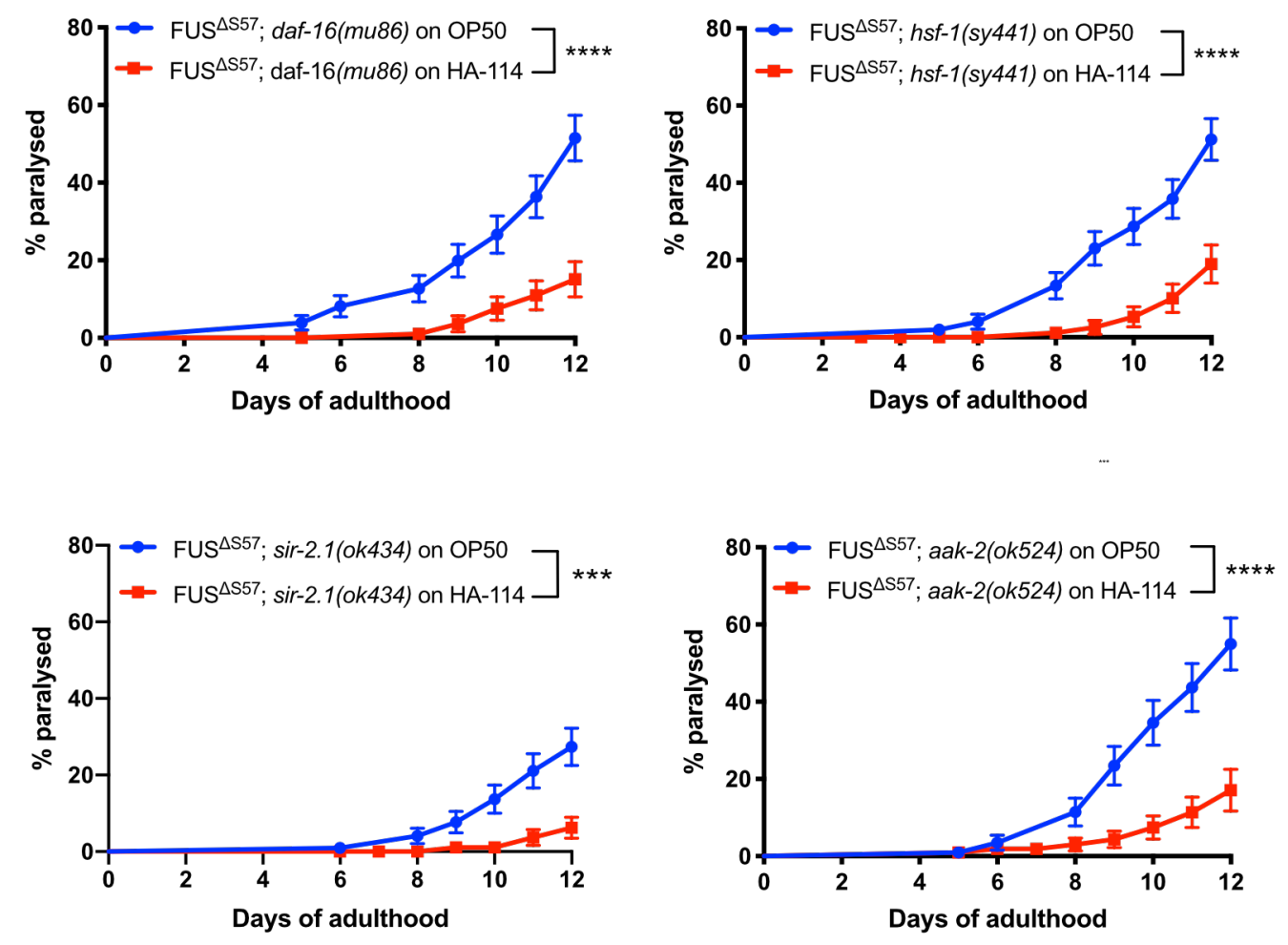

Figure S2. HA-114 does not require classic stress and metabolic pathways in FUS ${ }^{\Delta S 57}$ worms for neuroprotection. Transgenics were monitored from the adult stage, scored daily for paralysis. Neuroprotection provided by L. rhamnosus HA-114 was unaffected by daf-16(mu86) deletion, nor by hsf-1(sy441) point mutation. Both sir-2.1 and aak-2 genes are not required for neuroprotection granted by HA-114 probiotics. 
A

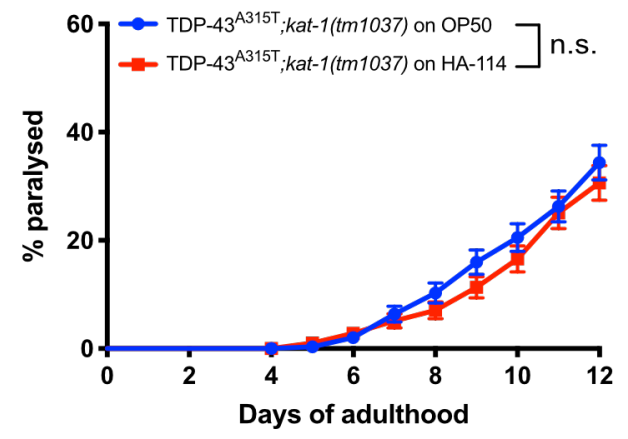

B

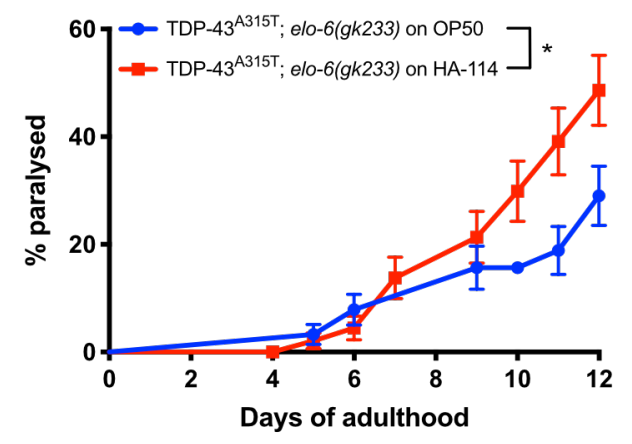

Figure S3. kat-1 and elo-6 are essential for neuroprotection provided by HA114 in TDP-43 ${ }^{A 315 T}$ ALS model. Transgenics were monitored from the adult stage, scored daily for paralysis. Deletion of kat-1 (A) and elo-6 (B) altered the beneficial effect of HA-114. *: $p<0.05$. 


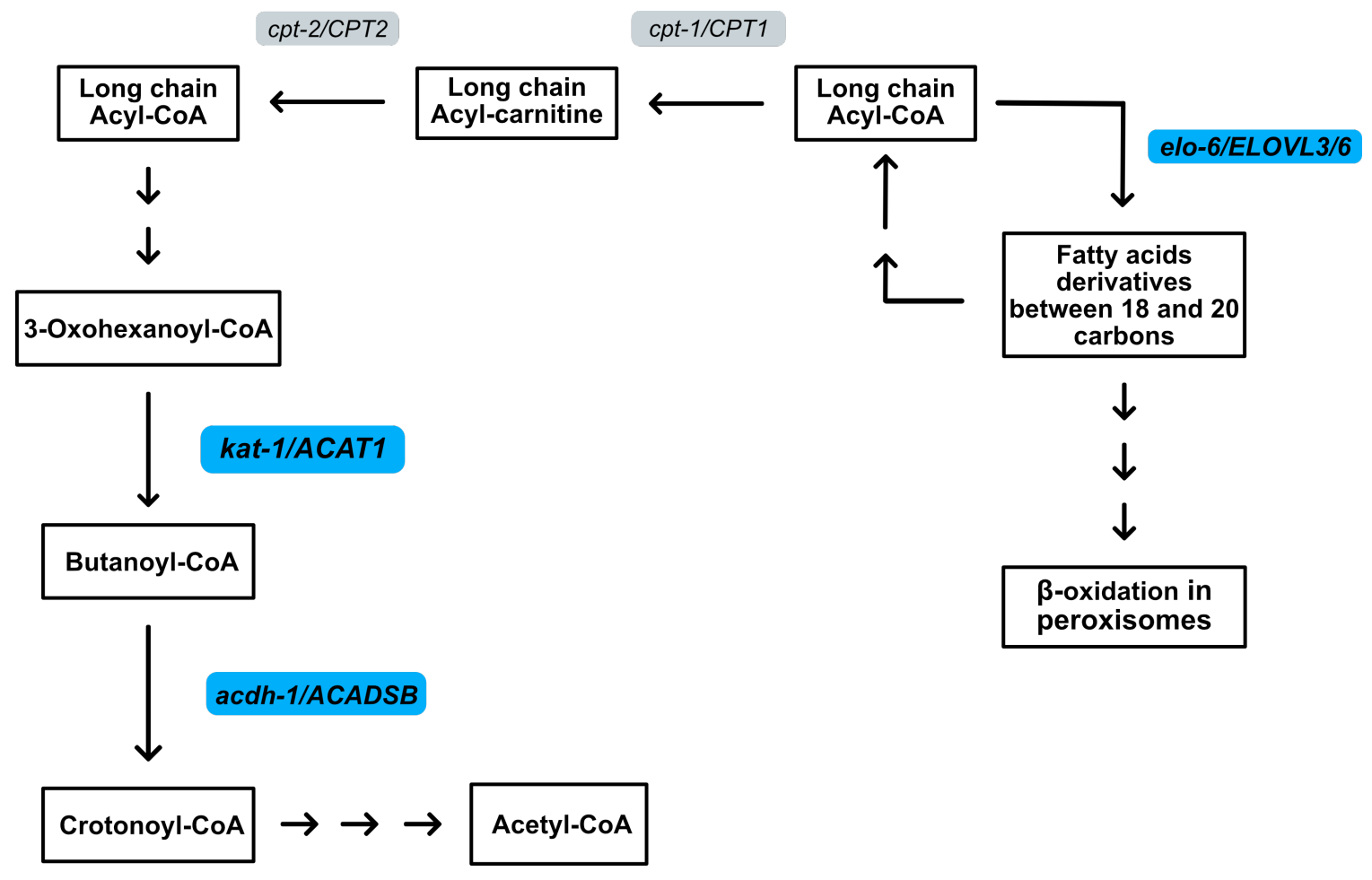

Figure S4 : Simplified pathway of fatty acids metabolism and $\beta$-oxidation in C. elegans and $\boldsymbol{H}$. sapiens. Enzymes names are enclosed in rounded rectangles. acdh-1/ACADSB: Acyl CoA dehydrogenase; cpt-1/CPT1: carnitine palmitoyl transferase 1; cpt-2/CPT2 : carnitine palmitoyl transferase 2; elo-6/ELOVL3/6: fatty acid elongase 3/6; kat-1/ACAT1: 3-Ketoacyl-coA thiolase. 
A
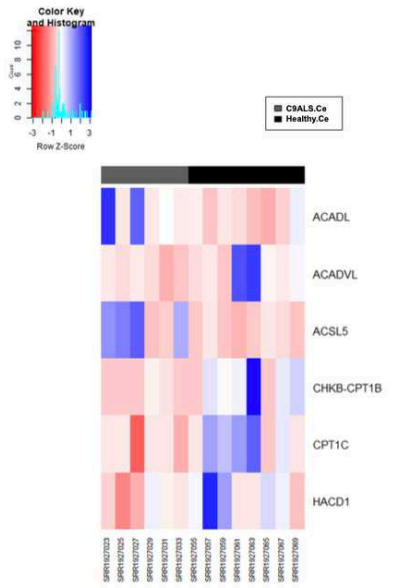

C

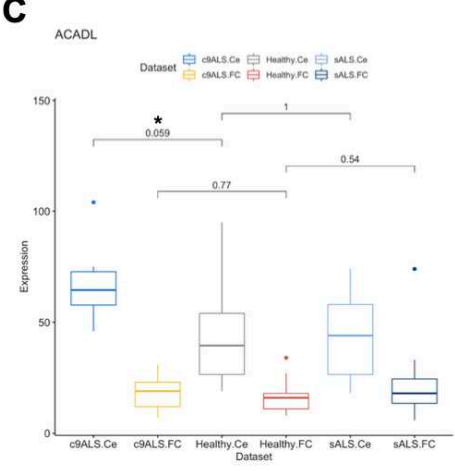

B

log2FoldChange c9ALS.Ce vs Heatthy.Ce
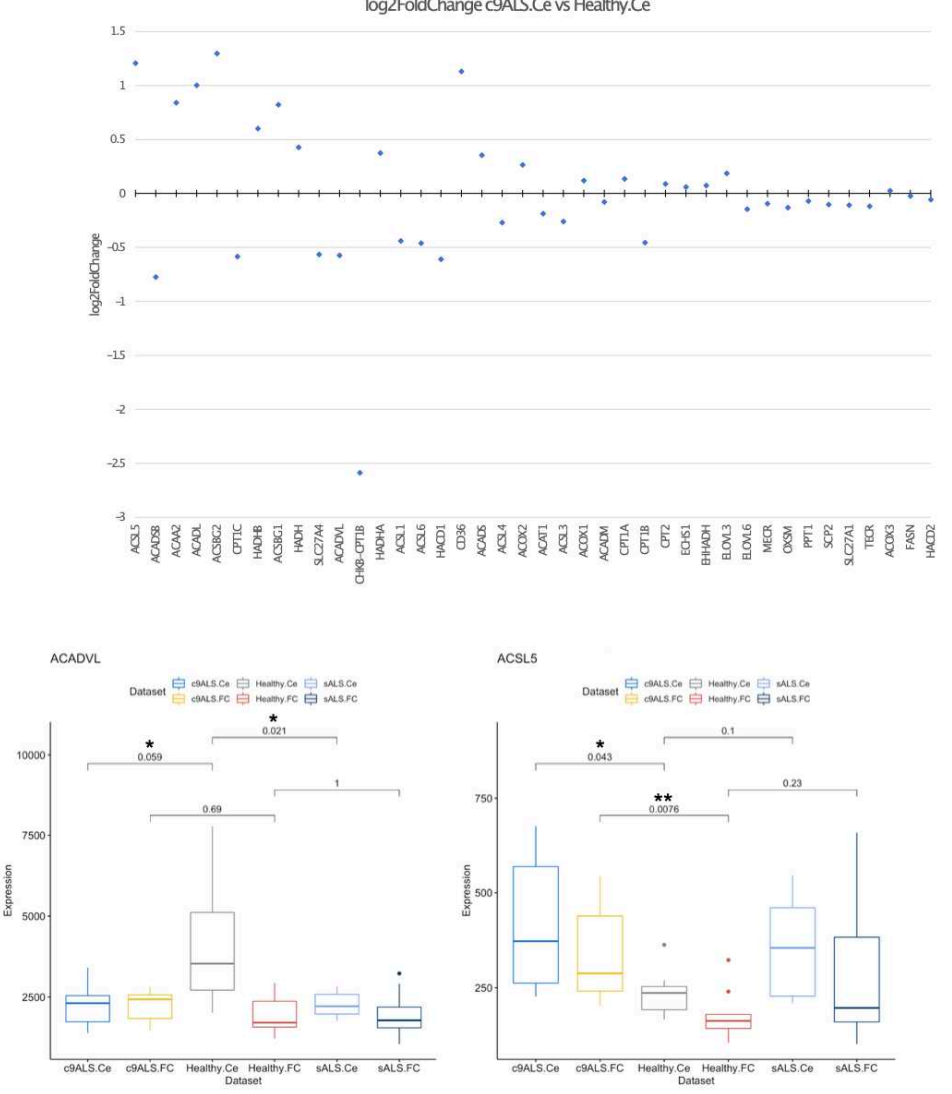

Figure S5: Genes associated with $\beta$-oxidation are differentially expressed in the cerebellum and the frontal cortex of c9ALS patients. (A) Differentially expressed genes associated with the $\beta$-oxidation pathway between C9orf72 ALS patients and healthy individuals were plotted as heatmap. Red indicates low relative expression and blue indicates high relative expression. (B) Log2 Fold change of differentially expressed genes associated with the $\beta$-oxidation pathway between C9orf72 ALS patients and healthy individuals. (C) ACADL expression is upregulated in the cerebellum of C9ALS and ACADVL is downregulated in C9ALS and SALS cerebellum. Interestingly, ACSL5 expression is upregulated in the cerebellum and the frontal cortex of c9ALS. Data: GEO accession: GSM1642314; SRA study: SRP056477. 
A

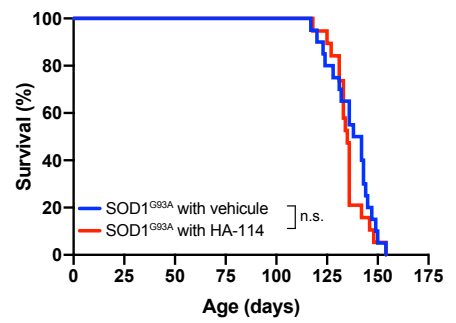

B

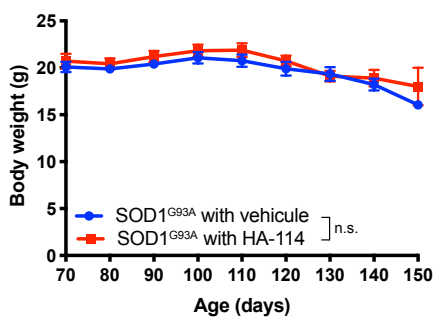

C

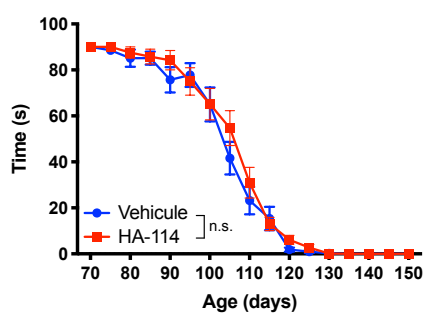

D

Neurological score
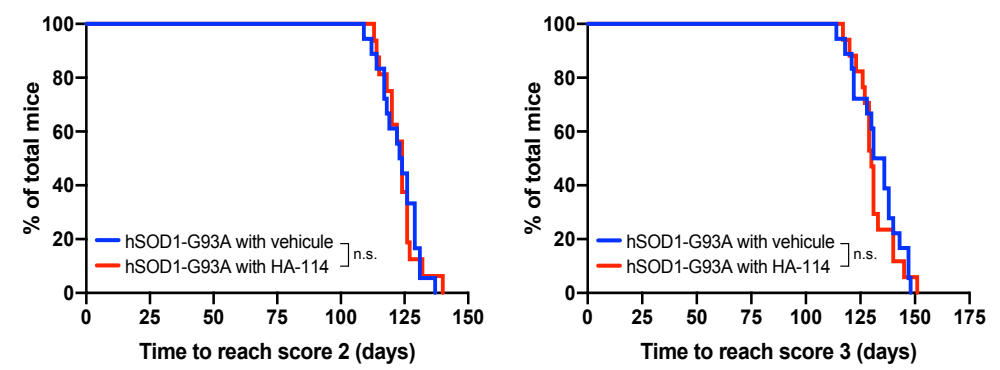

Figure S6. HA-114 does not extend lifespan in a SOD1 ${ }^{\text {G93A }}$ mouse model. Kaplan-Meier plot showing lifespan (A) Probiotics had no effect on body weight (B) hangwire assay (C) nor neurological score (D). 20 mice per group were used. 
A

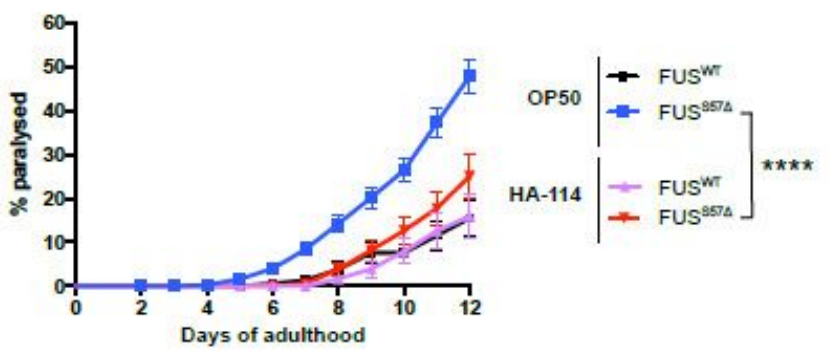

B

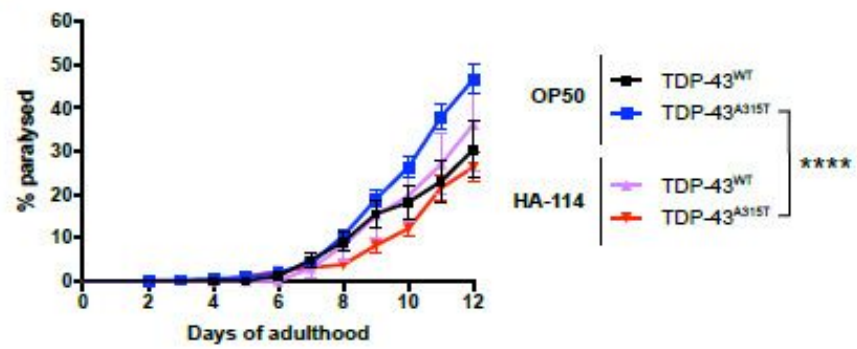

E

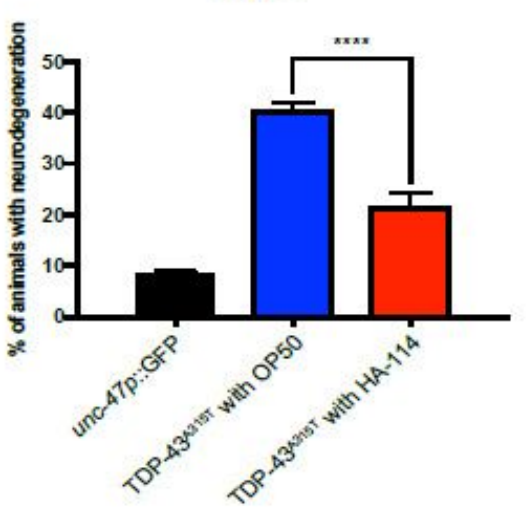

$\mathbf{F}$

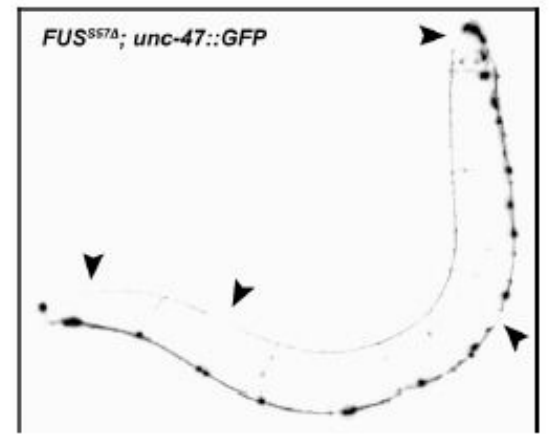

Day 9

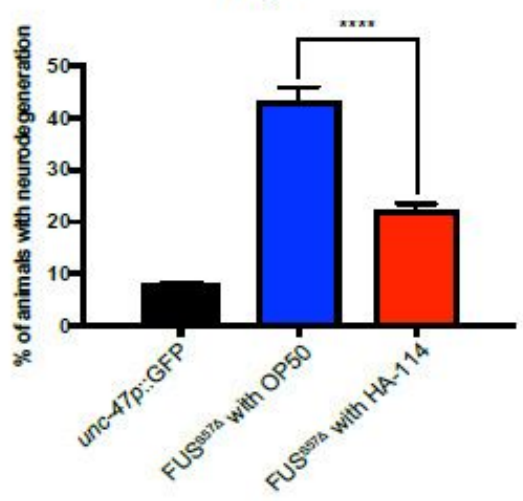

D

G

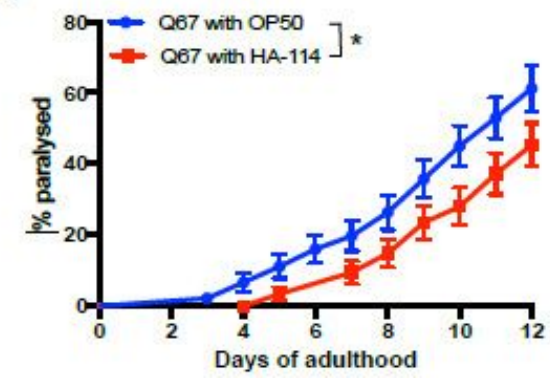

Figure 1

Lacticaseibacillus rhamnosus HA-114 rescues motor defects and neurodegeneration. Transgenics were monitored from the adult stage, scored daily for paralysis and fed with control OP50 or HA-114. (A) Mutant FUS worms fed with HA-114 showed less paralysis compared to transgenics expressing mutant FUS fed with OP50. (B) Transgenics expressing mutant TDP-43 fed with probiotics showed a lower rate of progressive paralysis than transgenics expressing mutant TDP-43 fed with OP50. (C) Image of a whole FUSDS57 worm expressing GFP in the GABAergic motor neurons. mFUS transgenics display gaps along neuronal processes (arrows). (D) Mutant FUS worms fed with HA-114 have a similar rate of neurodegeneration compared to transgenic GFP controls at day 9. (E) Mutant TDP-43 transgenics fed with probiotics had a lower rate of neurodegeneration at day 9 compared to mutant TDP-43 transgenics 
fed with OP50. (F) HA-114 rescued aged-dependent paralysis phenotype in transgenics expressing Q40 and in Q67 (G) animals. *: $p<0.05, * \star \star \star: ~ p<0.0001$.

A
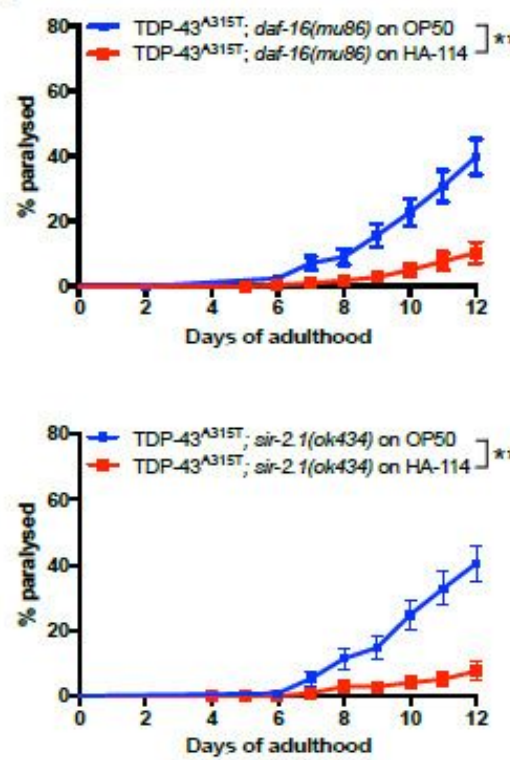

C

nIp-29::GFP

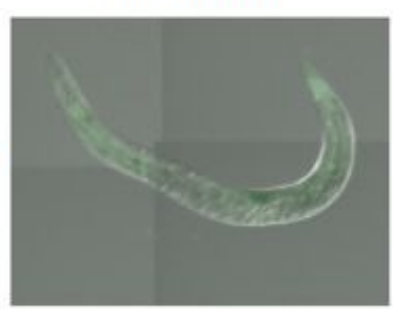

HA-114

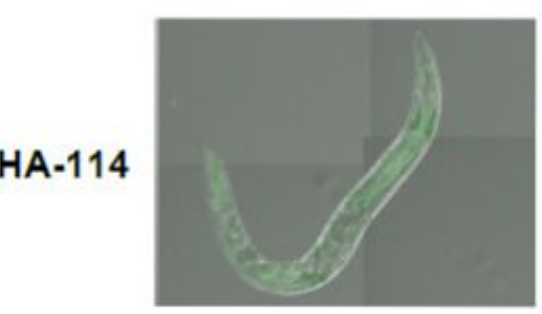

OP50
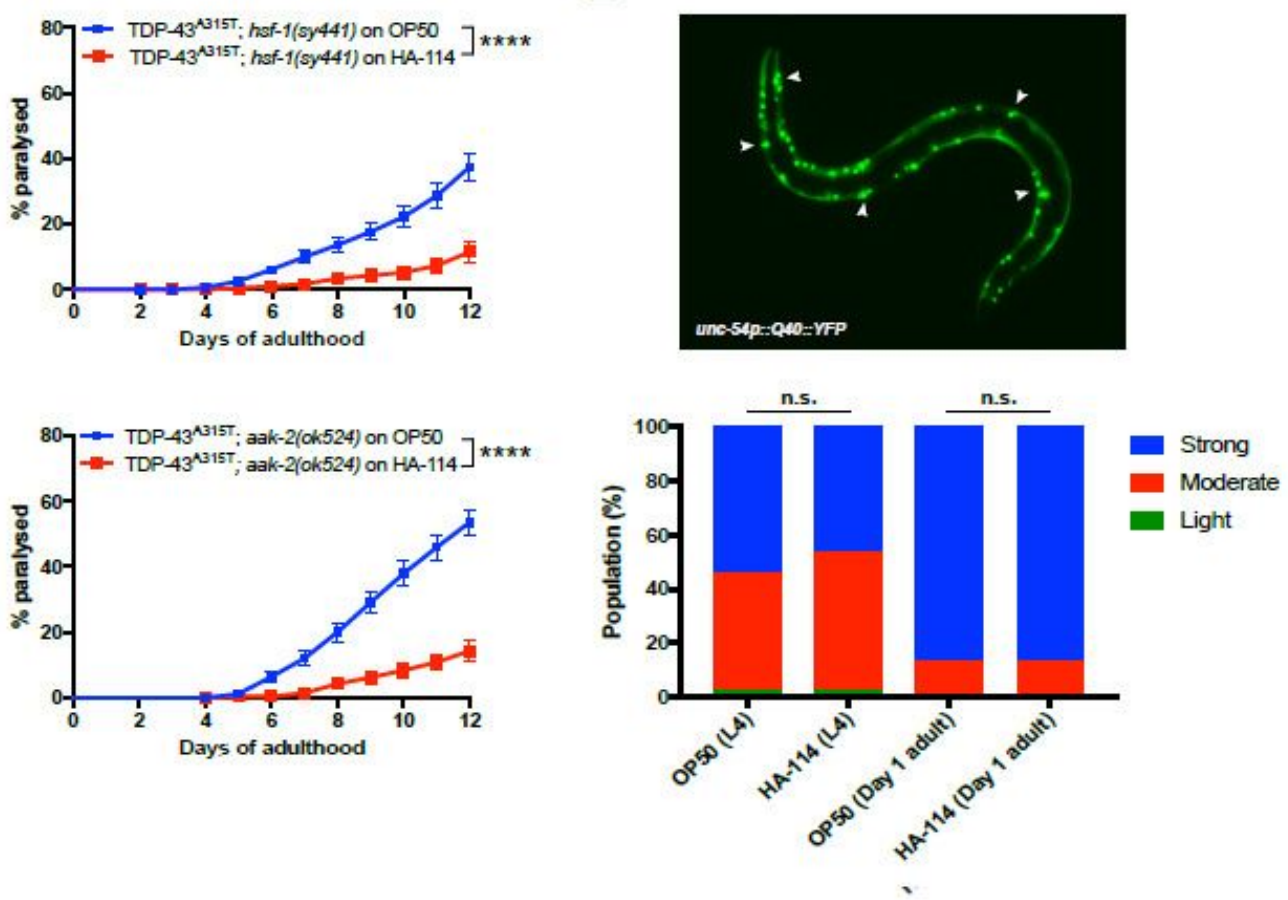

une-54p:Q40:YFP
hsp-6::GFP
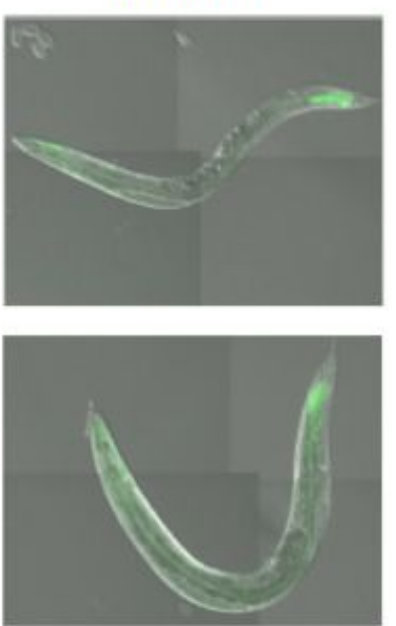

B

hsp-4::GFP
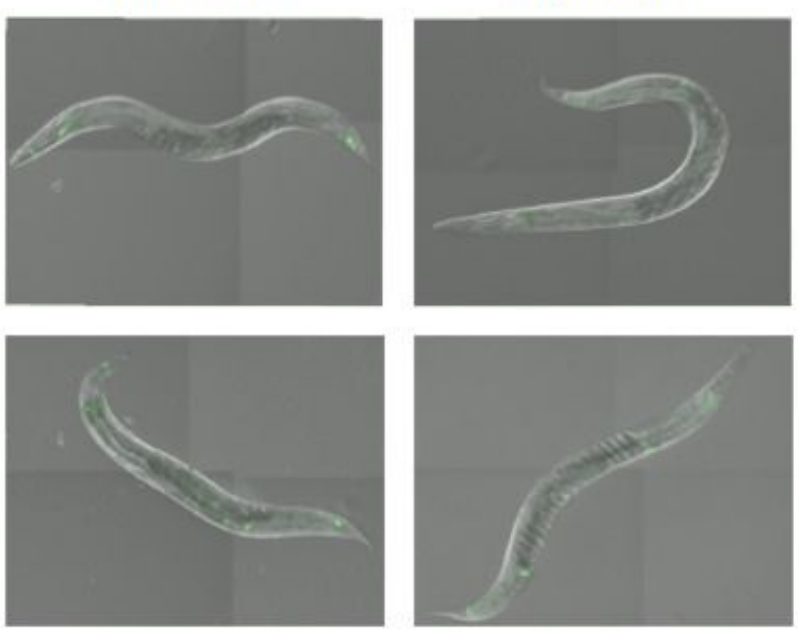

\section{Figure 2}

HA-114 does not require classic stress and metabolic pathways in C. elegans for neuroprotection. Neuroprotection provided by L. rhamnosus HA- 114 was unaffected by (A) daf-16(mu86) deletion, nor by hsf-1(sy441) point mutation. Both sir-2.1 and aak-2 genes are not required for neuroprotection granted by HA-114 probiotics. (B) Aggregation was not altered by HA-114 in a polyQ model at L4 or at day 1 of 
adulthood. (C) HA-114 did not affect GFP expression of key genes of innate immune response (nlp-29), UPRmito response (hsp-6 and dve-1) or UPRER (hsp-4).

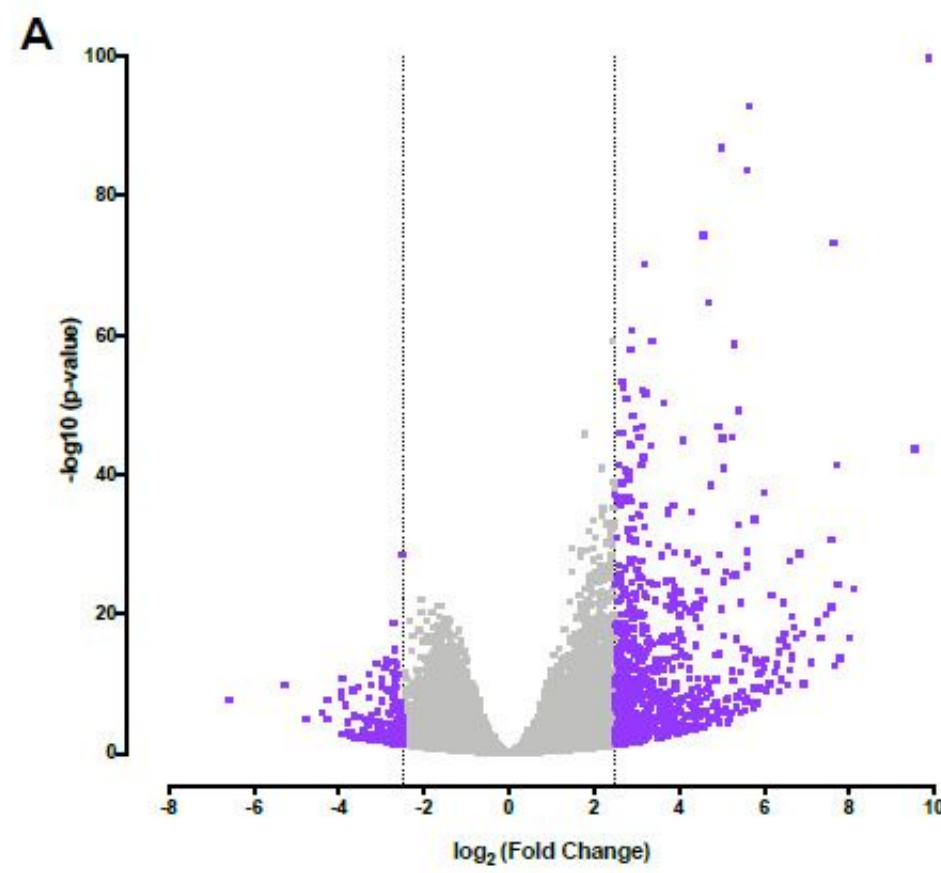

B
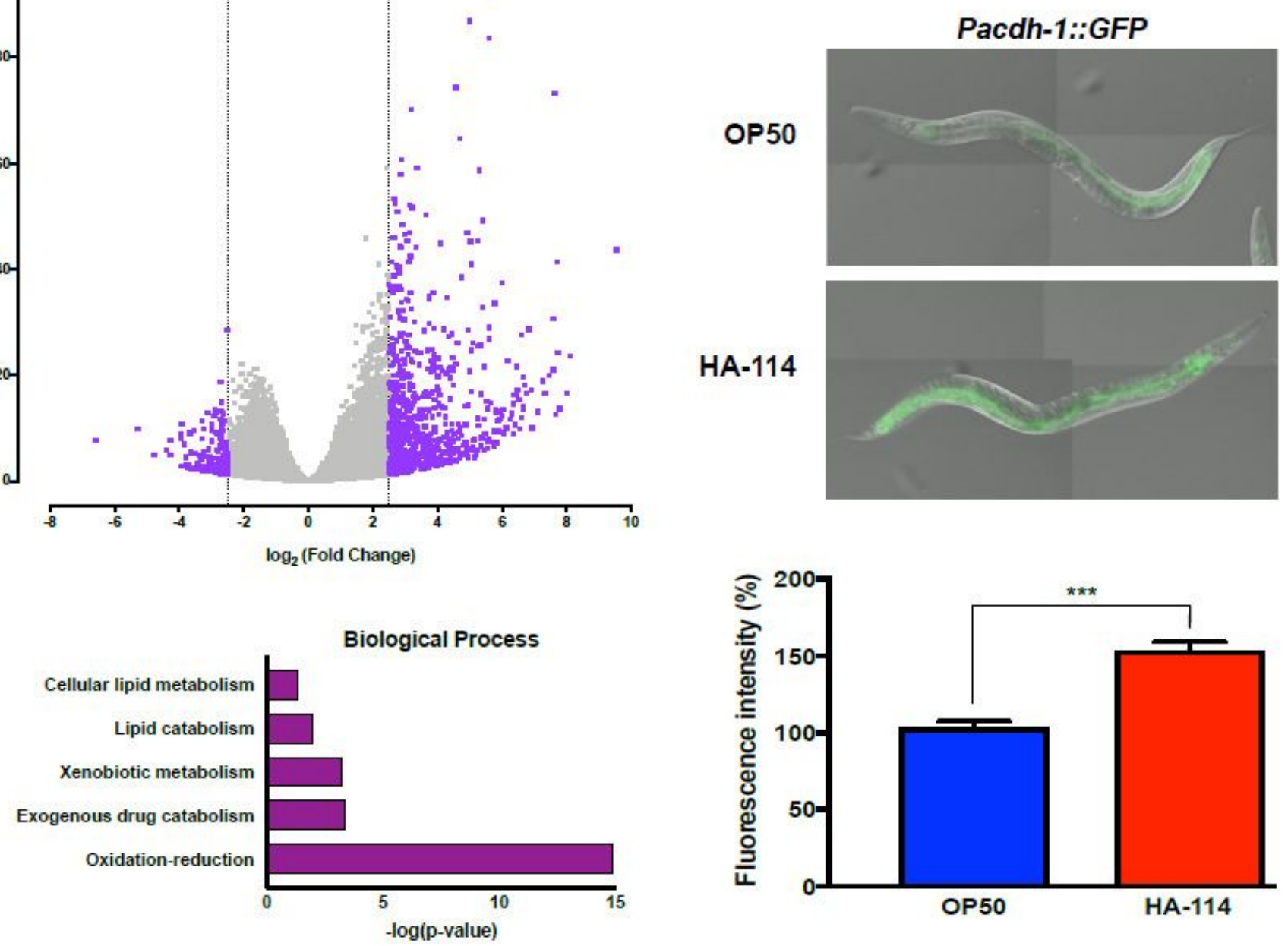

C

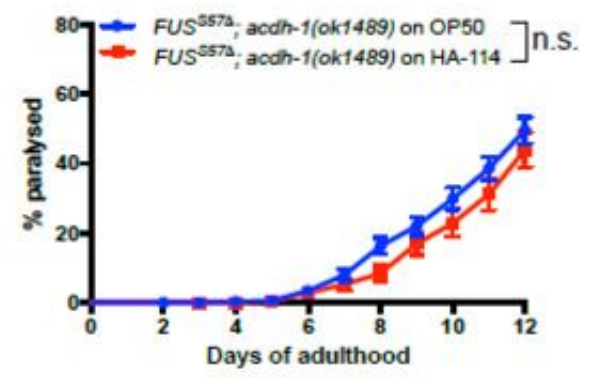

D

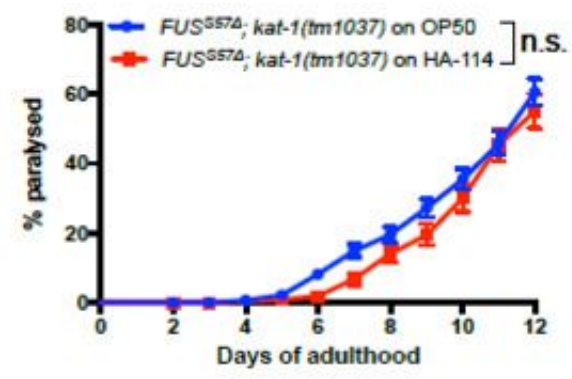

E

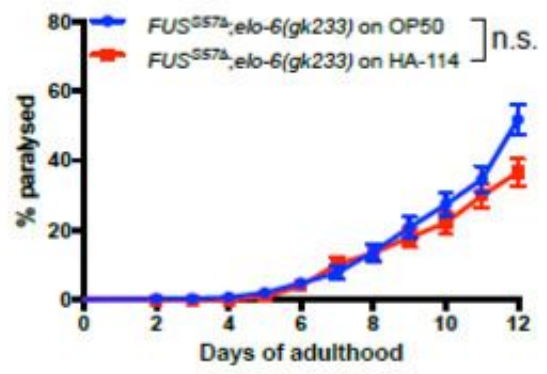

\section{Figure 3}

acdh-1, kat-1 and elo-6 are essential for neuroprotection provided by HA-114. (A) Volcano plot of RNA-Seq data of $\mathrm{N} 2$ worms fed with Lacticaseibacillus rhamnosus HA-114 and compared to worms fed with OP50. The data for all genes were plotted as log2 fold change versus - $\log 10$ of the adjusted p-value. Gene 
ontology (GO) term analysis of the differentially overexpressed genes induced by HA-114 treatment. The GO term analysis was performed with PANTHER 11 by using C. elegans genes as background. Only the biological process terms with an enrichment of $P$-value $<0.01$ are shown in this figure. (B) L. rhamnosus HA-114 significantly increase GFP expression in pacdh-1::GFP worms when compared to the same worms fed with OP50. Deletion of acdh-1 (C), kat-1 (D) and elo-6 (E) altered the beneficial effect of HA114. ***: $p<0.001$.

A

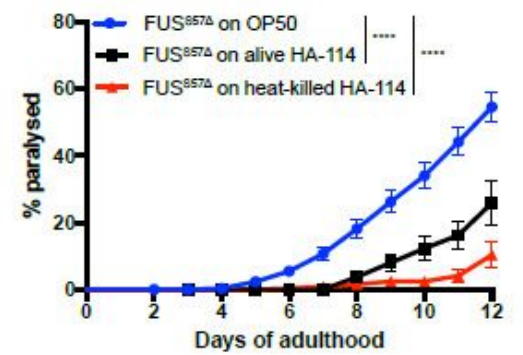

D

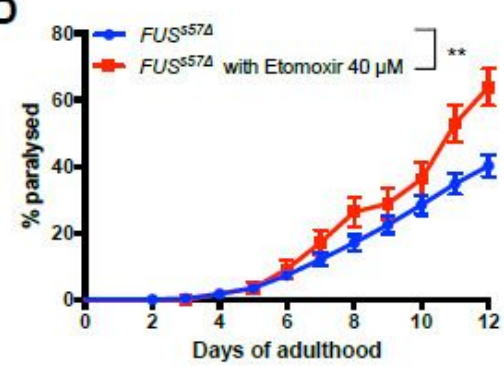

$\mathbf{F}$

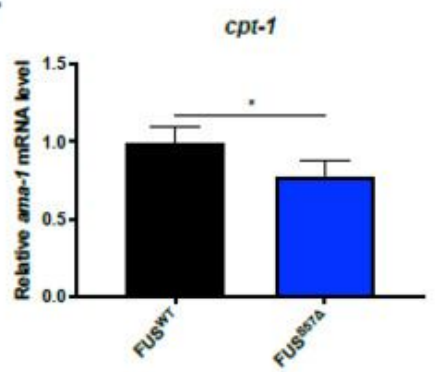

H

CHKA CPT1E

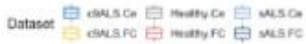

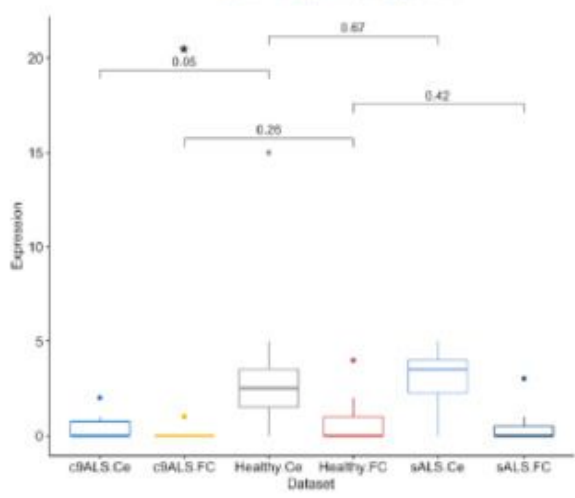

B

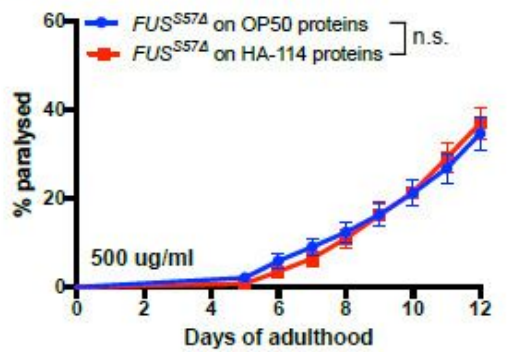

E

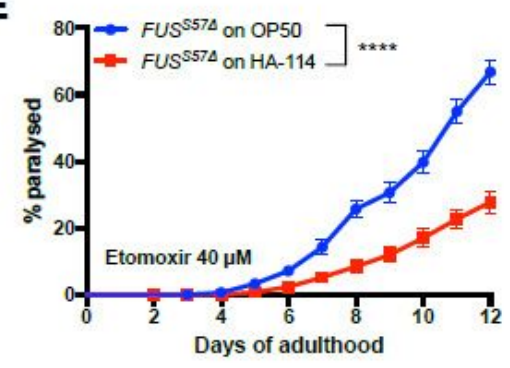

G

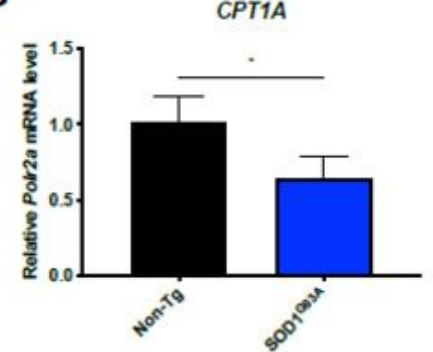

E
C

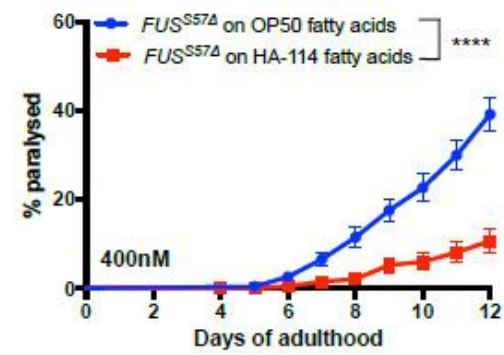

Days of adulthood

.


HA-114 fatty acids extracts, but not proteins, are sufficient to rescue paralysis. Transgenics were monitored from the adult stage, scored daily for paralysis and fed with control OP50 or HA-114's individual components. (A) Mutant FUS worms fed with heat-killed L. rhamnosus HA-114 did not show paralysis phenotypes when compared to worms fed with OP50. (B) Protein extract from L. rhamnosus HA-114 failed to rescue age-dependent paralysis in ALS worm models. (C) When compared to fatty acid extract from OP50, fatty acids extract from HA-114 suppressed paralysis phenotypes in FUS animals. (D) $40 \mu \mathrm{M}$ of Etomoxir, a cpt-1/CPT1 inhibitor, increased paralysis in FUS transgenics. (E) The same concentration was not enough to block neuroprotective effects of $L$. rhamnosus HA-114, when compared to worms fed with OP50. (F) mRNA expression of cpt- 1 was significantly decreased in FUSDS57 worms when compared to FUSWT animals. (G) mRNA expression of CPT1A was significantly decreased in livers of SOD1G93A mice when compared to livers of Non-Tg animals. $(H)$ Decrease expression of two transcripts related to carnitine palmitoyltransferase (CHKB_CPT1B and CPT1C) is observed in C9ORF72 patients when compared to controls. No significant change is observed in sporadic ALS. Data: GEO accession: GSM1642314; SRA study: SRP056477. (Prudencio et al., 2015). *: $p<0.05, * *: p<0.01, * \star \star *: p<$ 0.0001 .

A

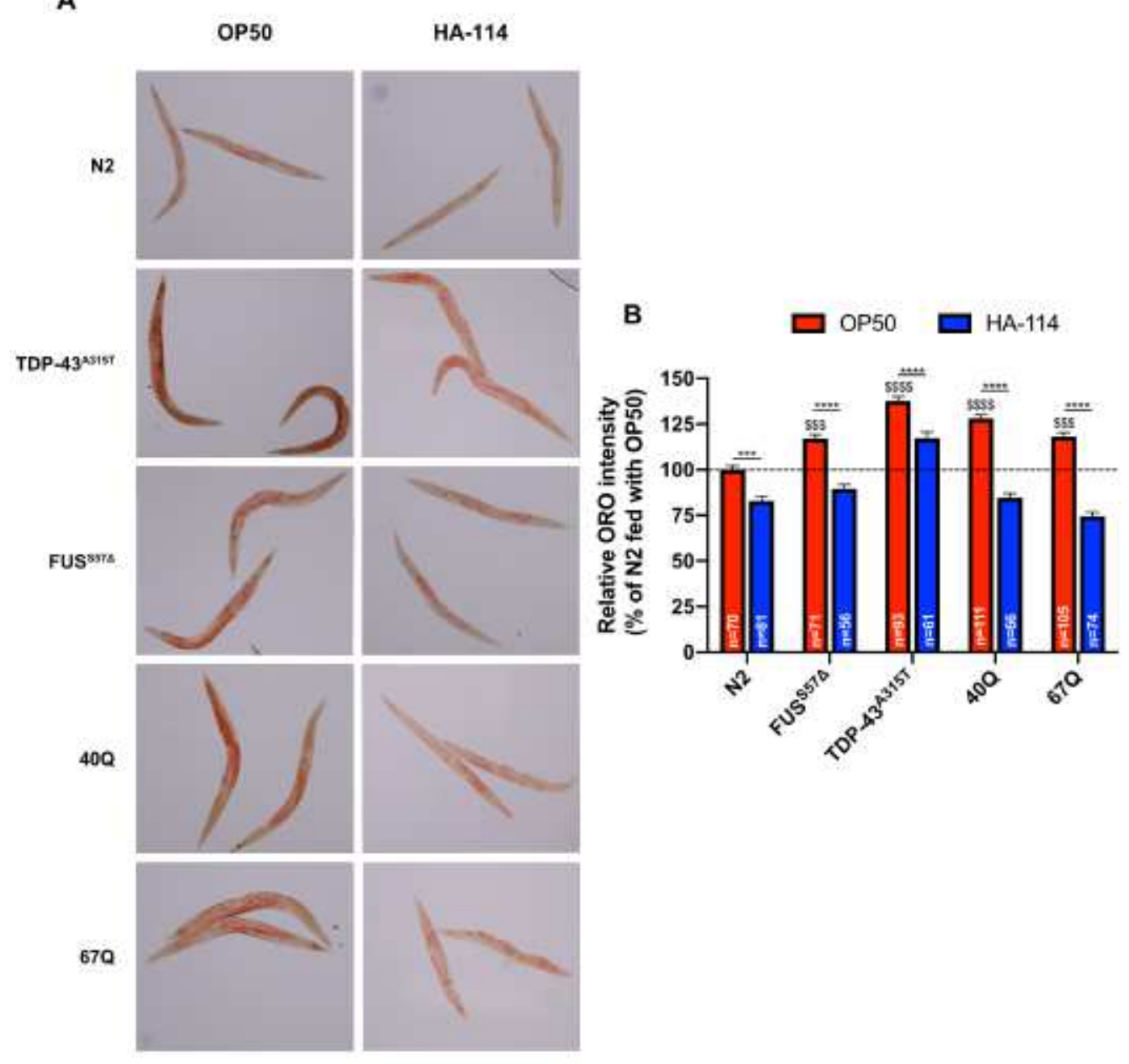

Figure 5 
Lacticaseibacillus rhamnosus HA-114 modulate lipid accumulation in worm age-dependent neurodegeneration models. (A) Representative images of worms fed with OP50 and HA-114 and stained with Oil Red O. N2 worms show a basal level of lipid accumulation. (B) Quantification of Oil Red O show increased fat accumulation in worms expressing FUSDS57, TDP-43A315T, Ab1-42 and polyQ expansion (40Q and 67Q) when compared to N2. Worms fed with HA-114 showed a significant decrease of lipid accumulation. \$: statistical difference when compared to N2, *: statistical difference within group. *** or

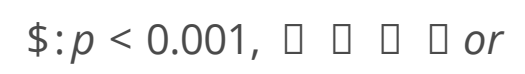

$\$ \$: p<0.0001$.

A

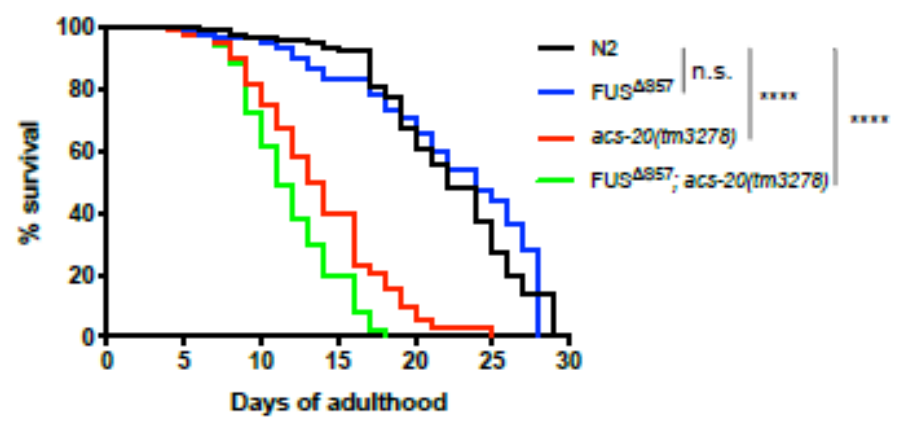

C

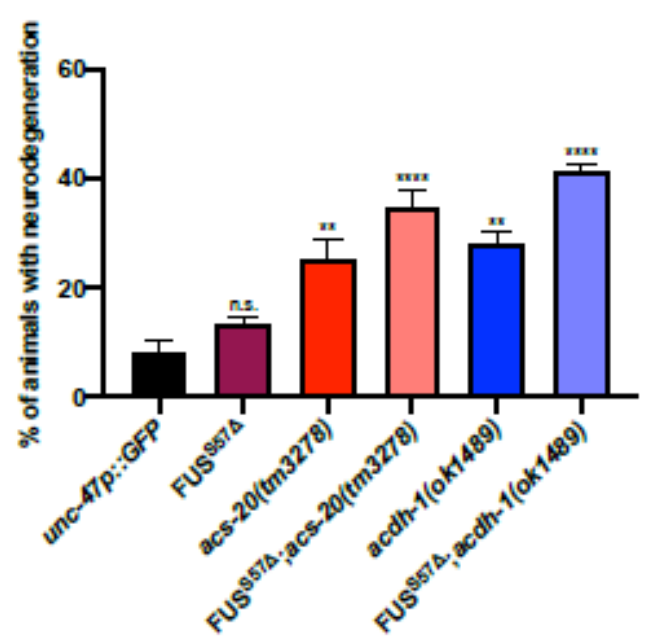

B

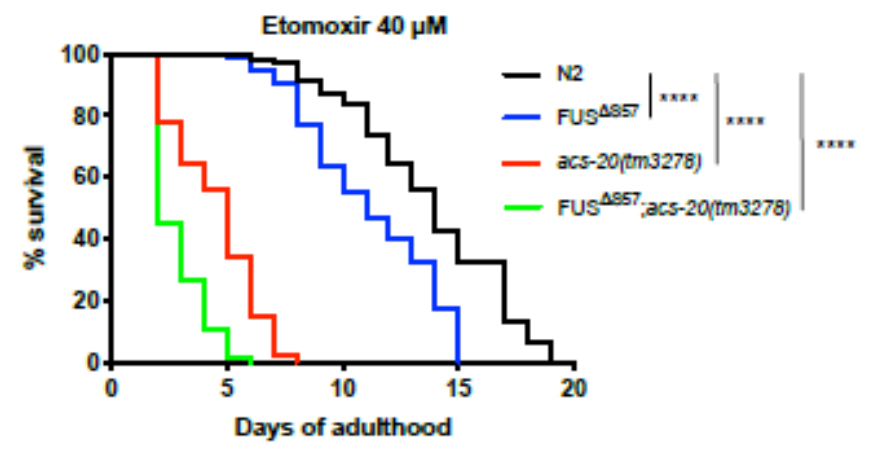

\section{Figure 6}

Impaired b-oxidation contributes to neurodegeneration and shortens lifespan. Worms were monitored from the adult stage until death. (A) acs-20(tm3278) and FUSDS57;acs-20(tm3278) worms have shorter lifespan than N2 and FUSDS57 animals. (B) Etomoxir (40 $\mu \mathrm{M})$, a cpt-1/CPT1 inhibitor, shorten lifespan of FUSDS57, acs-20(tm3278) and FUSDS57;acs-20(tm3278) worms when compared to N2. (C) acs20(tm3278), FUSDS57;acs-20(tm3278), acdh-1(ok1489) and FUSDS57;acdh-1(ok1489) animals have a 
higher rate of neurodegeneration compared to transgenic GFP controls at day 1. **: $p<0.01,{ }^{\star \star *}: p<$ 0.0001 .

A

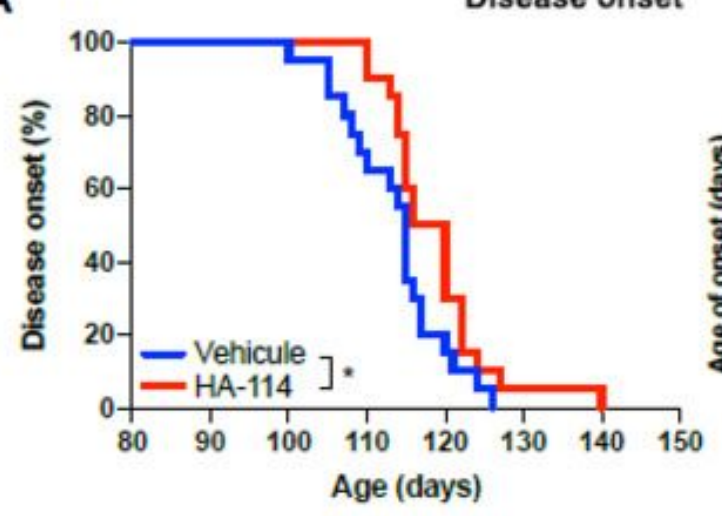

C
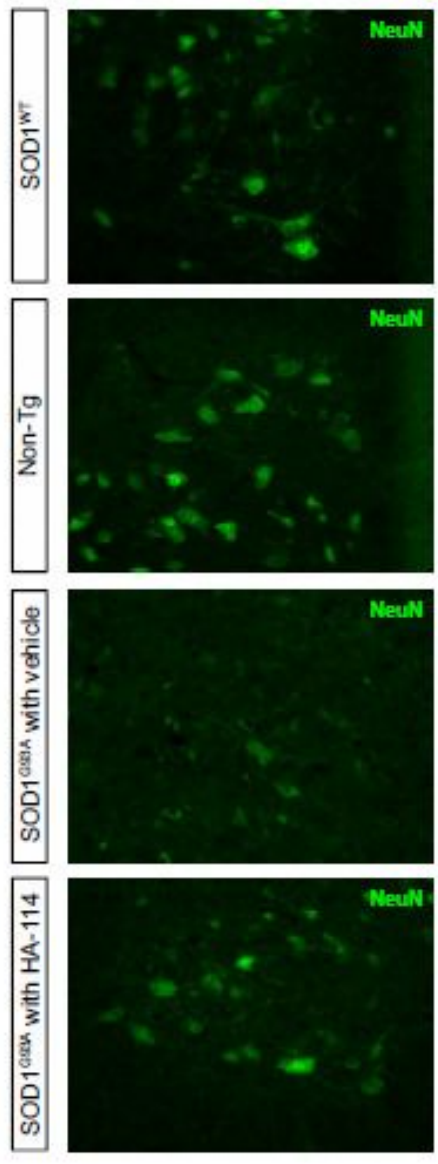
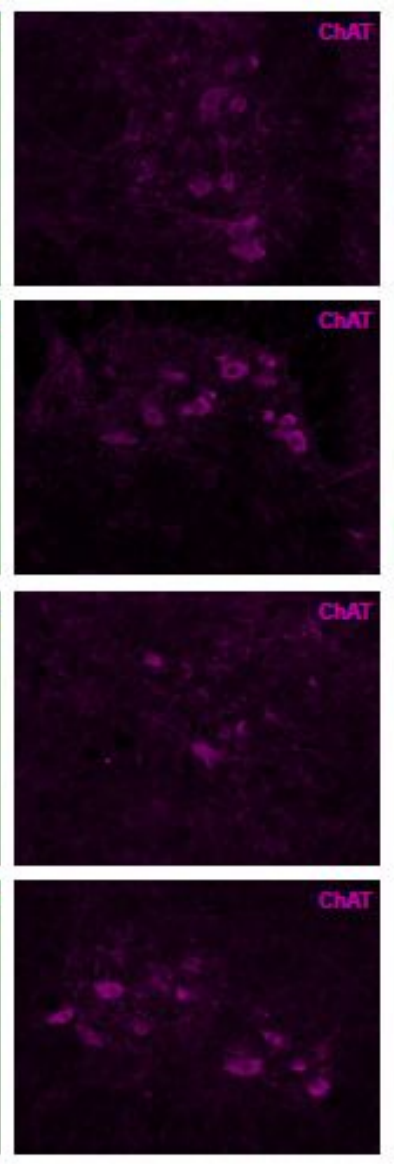

B
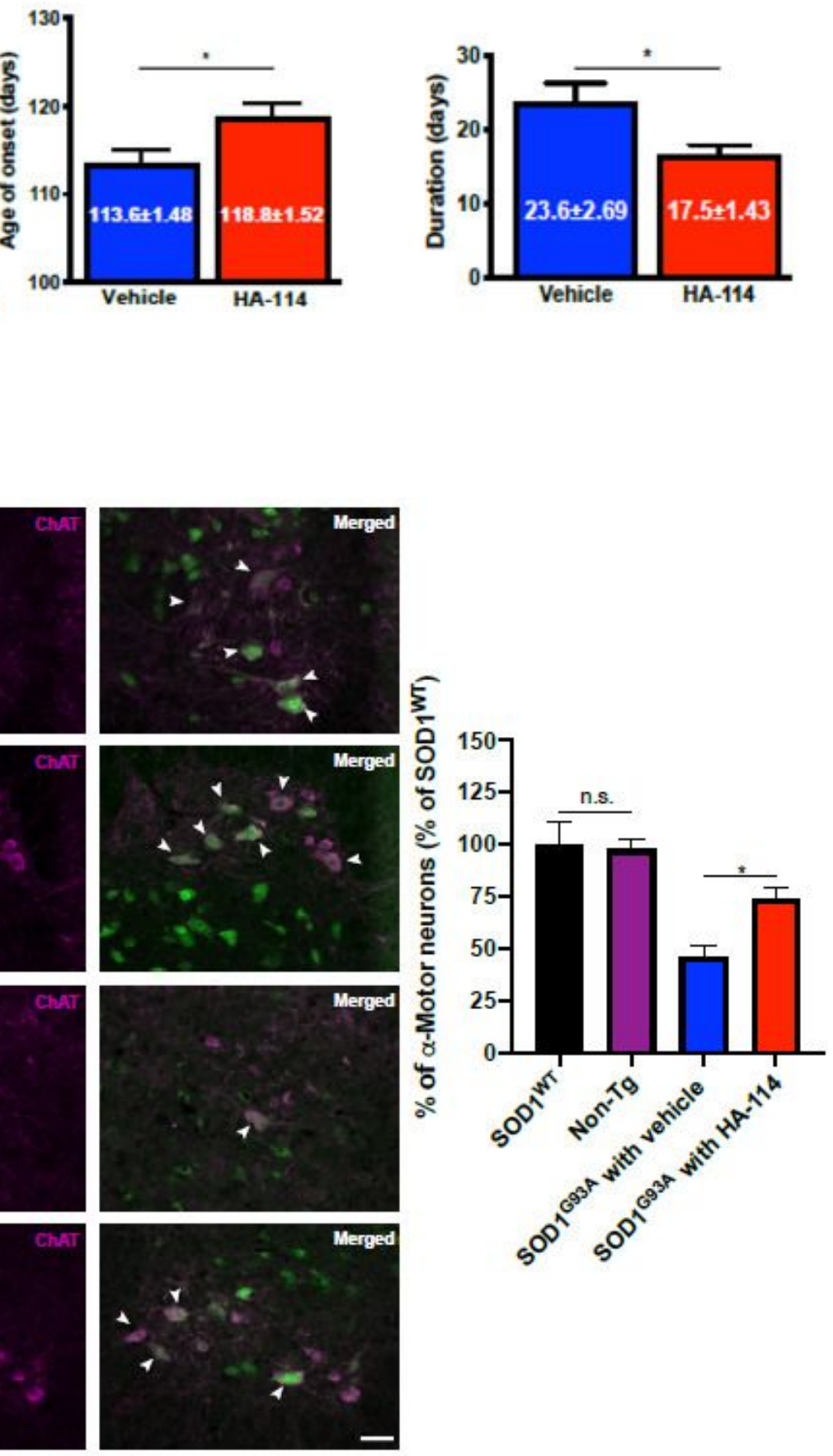

Figure 7

HA-114 delays disease onset in a SOD1G93A mouse model. Kaplan- Meier plot and graph bar showing onset of the disease (A). Onset is delayed in mice treated with $L$. rhamnosus HA-114 (vehicle : 113.6 1.48 days, HA-114: $118.8 \pm 1.52$ days). (B) Disease duration is also decreased in the group treated with HA-114 
(vehicle: $23.6 \pm 2.69$ days, HA-114: $17.5 \pm 1.43$ days). 20 mice per group were used. (C) Images of immunofluorescence labeling of motor neurons in the ventral horn of lumbar spinal cord sections; neuronal-specific nuclear protein (NeuN: green) and choline acetyltransferase (ChAT; magenta) were labelled for four different experimental groups at P120 (SOD1WT, Non-Tg, SOD1G93A with vehicle and SOD1G93A with HA-114). Graph bar shows the percentage of double-positive cells (NeuN+/ChAT+), normalized to SOD1WT values. HA-114 treatment significantly improved motor neurons loss in SOD1G93A mice. Scale bar: $50 \mu \mathrm{m}$. *: $p<0.05$.

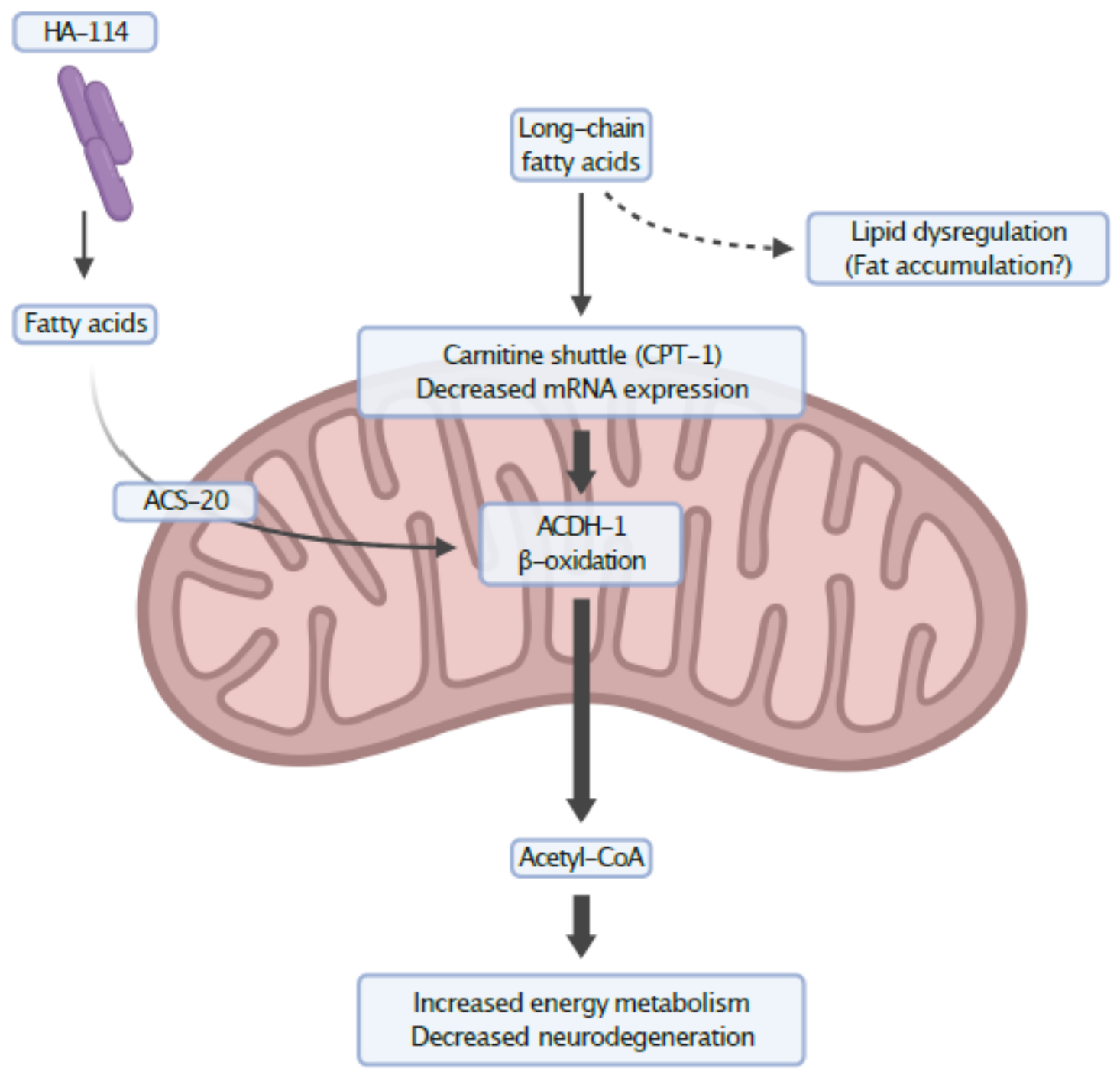

\section{Figure 8}

Neuroprotection mechanism of Lacticaseibacillus rhamnosus HA-114. ALS models have impaired carnitine shuttle, a mechanism to transport long chain fatty acids across the mitochondrial membrane for energy production via boxidation. Fatty acids, supplied by the probiotic bacteria, is believe to enter the mitochondria independently of the carnitine shuttle to participate in a few rounds of b-oxidation helping 
to stabilize energy metabolism, resulting in decreased neurodegeneration and improved lipid homeostasis.

\section{Supplementary Files}

This is a list of supplementary files associated with this preprint. Click to download.

- Figs1.png

- Figs2.png

- FigS3.png

- Figs4.png

- Figs5.png

- Figs6.png 